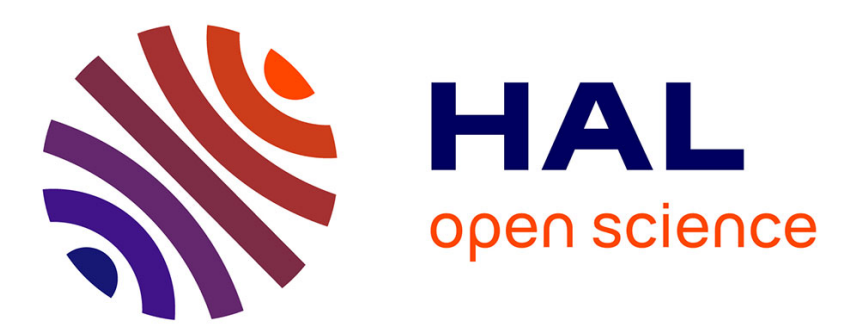

\title{
Gradient discretization of hybrid-dimensional Darcy flow in fractured porous media with discontinuous pressures at matrix-fracture interfaces
}

Konstantin Brenner, Julian Hennicker, Roland Masson, Pierre Samier

\section{- To cite this version:}

Konstantin Brenner, Julian Hennicker, Roland Masson, Pierre Samier. Gradient discretization of hybrid-dimensional Darcy flow in fractured porous media with discontinuous pressures at matrixfracture interfaces. IMA Journal of Numerical Analysis, 2016, 10.1093/imanum/drw044 . hal01192740v2

\author{
HAL Id: hal-01192740 \\ https://hal.science/hal-01192740v2
}

Submitted on 9 Aug 2016

HAL is a multi-disciplinary open access archive for the deposit and dissemination of scientific research documents, whether they are published or not. The documents may come from teaching and research institutions in France or abroad, or from public or private research centers.
L'archive ouverte pluridisciplinaire $\mathbf{H A L}$, est destinée au dépôt et à la diffusion de documents scientifiques de niveau recherche, publiés ou non, émanant des établissements d'enseignement et de recherche français ou étrangers, des laboratoires publics ou privés. 


\title{
Gradient discretization of hybrid dimensional Darcy flows in fractured porous media with discontinuous pressures at the matrix fracture interfaces
}

\author{
K. Brenner*, J. Hennicker*,†, R. Masson*, P. Samier ${ }^{\dagger}$
}

August 8, 2016

\begin{abstract}
We investigate the discretization of Darcy flow through fractured porous media on general polyhedral meshes. We consider a hybrid dimensional model, invoking a complex network of planar fractures. The model accounts for matrix-fracture interactions and fractures acting either as drains or as barriers, i.e. we have to deal with pressure discontinuities at matrix-fracture interfaces. The numerical analysis is performed in the general framework of gradient discretizations which is extended to the model under consideration. Two families of schemes namely the Vertex Approximate Gradient scheme (VAG) and the Hybrid Finite Volume scheme (HFV) are detailed and shown to fit in the gradient scheme framework, which yields, in particular, convergence. Numerical tests confirm the theoretical results. Gradient Discretization; Darcy Flow, Discrete Fracture Networks, Finite Volume
\end{abstract}

\section{Introduction}

This work deals with the discretization of Darcy flows in fractured porous media for which the fractures are modelized as interfaces of codimension one. In this framework, the $d-1$ dimensional flow in the fractures is coupled with the $d$ dimensional flow in the matrix leading to the so called, hybrid dimensional Darcy flow model. We consider the case for which the pressure can be discontinuous at the matrix fracture interfaces in order to account for fractures acting either as drains or as barriers as described in [18], [21] and [4]. In this paper, we will study the family of models described in [21] and [4].

It is also assumed in the following that the pressure is continuous at the fracture intersections. This corresponds to a ratio between the permeability at the fracture intersection and the width of the fracture assumed to be large compared with the ratio between the tangential permeability of each fracture and its length. We refer to [19] for a more general reduced model taking into account discontinuous pressures at fracture intersections in dimension $d=2$.

The discretization of such hybrid dimensional Darcy flow models has been the object of several works. In [18], [20], [4] a cell-centered Finite Volume scheme using a Two Point Flux

*Laboratoire de Mathématiques J.A. Dieudonné, UMR 7351 CNRS, University Nice Sophia Antipolis, and team COFFEE, INRIA Sophia Antipolis Méditerranée, Parc Valrose 06108 Nice Cedex 02, France, \{konstantin.brenner, julian.hennicker, roland.masson\}@unice.fr

†CSTJF, TOTAL S.A. - Avenue Larribau, 64018 Pau, France 
Approximation (TPFA) is proposed assuming the orthogonality of the mesh and isotropic permeability fields. Cell-centered Finite Volume schemes using MultiPoint Flux Approximations (MPFA) have been studied in [28], [26], and [2]. In [21], a Mixed Finite Element (MFE) method is proposed and a MFE discretization adapted to non-matching fracture and matrix meshes is studied

in [10]. More recently the Hybrid Finite Volume (HFV) scheme, introduced in [15], has been extended in [17] for the non matching discretization of two reduced fault models. Also a Mimetic Finite Difference (MFD) scheme is used in [5] in the matrix domain coupled with a TPFA scheme in the fracture network. Discretizations of the related reduced model [3] assuming a continuous pressure at the matrix fracture interfaces have been proposed in [3] using a MFE method, in [23] using a Control Volume Finite Element method (CVFE), in [7] using the HFV scheme, and in $[7,6]$ using an extension of the Vertex Approximate Gradient (VAG) scheme introduced in [14].

In terms of convergence analysis, the model assuming continuous pressure at the matrix fracture interfaces [3] is studied in [7] for a general fracture network but the current state of the art for the discontinuous pressure models is still limited to rather simple geometries. Let us recall that the family of models introduced in [21] and [4] depends on a quadrature parameter denoted by $\xi \in\left[\frac{1}{2}, 1\right]$ for the approximate integration in the width of the fractures.

For such models, convergence of MFE discretizations in [21] and non matching MFE discretizations in [10] for the range $\xi \in\left(\frac{1}{2}, 1\right]$ has been established in the case of a single fracture separating the matrix domain into two subdomains. In [4], the case of one fully immersed fracture in dimension $d=2$ using a TPFA discretization is analysed for the full range of parameters $\xi \in\left[\frac{1}{2}, 1\right]$.

The main goal of this paper is to study the discretization of such models and provide the first proof of convergence for a general fracture network in a 3D surrounding matrix domain including fully, partially and non immersed fractures as well as fracture intersections. Each individual fracture will be assumed to be planar and our analysis will cover the range of parameters $\xi \in\left(\frac{1}{2}, 1\right]$. The value $\xi=\frac{1}{2}$ is excluded in order to allow for a primal variational formulation. This objective will be achieved by extension to the hybrid dimensional Darcy flow model of the gradient scheme framework introduced in [14], [12]. The gradient scheme framework allows to analyze the convergence of both conforming and non conforming discretizations for linear and nonlinear second order diffusion and parabolic problems. As shown in [12], it accounts for various schemes such as Finite Element methods, Mixed and Mixed Hybrid Finite Element methods, some Finite Volume schemes like symmetric MPFA, the VAG schemes [14], and the HFV schemes [15]. The main advantage of this framework is to provide the convergence proof for all schemes satisfying some abstract conditions, namely coercivity, consistency and limit conformity, at the price of a single convergence analysis for a given model.

The second objective of this paper is to prove the coercivity, consistency and limit conformity properties for two examples of gradient discretizations, namely the extension of the VAG and HFV schemes defined in [14] and [15] to the family of hybrid dimensional Darcy flow models. The proof of these properties is as usual based on a key result proved in the appendix and stating the density of smooth functions subspaces in both the variational space and in the flux space of the model. The mesh is assumed to be polyhedral with possibly non planar faces for the VAG scheme and planar faces for the HFV scheme. It is assumed that the fracture network is conforming to the mesh in the sense that it is defined as a collection of faces of the mesh. Two versions of the VAG scheme will be studied, the first corresponding to the conforming $\mathbb{P}_{1}$ finite element on a tetrahedral submesh, and the second to a finite volume scheme using lumping for the source terms as well as for the matrix fracture fluxes. Note that the HFV scheme of [15] has been generalized in [13] as the family of Hybrid Mimetic Mixed methods which which 
encompasses the family of MFD schemes [9]. In this article, we will focus without restriction on the particular case presented in [15] for the sake of simplicity.

Our third objective is to compare numerically the VAG and HFV discretizations in terms of accuracy and CPU time on Cartesian, hexahedral and tetrahedral families of meshes. For that, an original family of analytical solutions is built in the case of four intersecting fractures, which takes into account heterogeneity and anisotropy of the medium. The HFV scheme uses cell, face and fracture edge unknowns, while the VAG scheme uses cell, node, and fracture face unknowns. In both cases, the cell unknowns can be eliminated without any fill-in before solving the linear system. As a remainder, MPFA schemes use cell and fracture face unknowns as well as edge unknowns at fracture intersections (see [28], [26], and [2]). Also, except on tetrahedral and parallepipedic meshes, MPFA schemes are non symmetric and their coercivity is conditioned to the mesh and to the anisotropy of the permeability fields. The VAG scheme has the advantage, compared with the HFV or MPFA schemes, to lead to a much sparser discretization on tetrahedral or triangular prismatic meshes, since the number of nodes is much smaller than the number of faces or cells for such meshes. Another advantage of the VAG scheme compared with the HFV scheme is to allow for non planar faces. The numerical experiments show that the VAG scheme provides a better compromise between accuracy and CPU time as well as a better robustness with respect to the anisotropy of the medium.

In section 2 we introduce the geometry of the matrix and fracture domains and present the strong and weak formulations of the model. Section 3 is devoted to the introduction of the of gradient discretization framework and the derivation of the error estimate. In section 4 we introduce and investigate the families of VAG and HFV discretizations. In section 5, the VAG and HFV schemes are compared in terms of accuracy and CPU efficiency for Cartesian, hexahedral and tetrahedral meshes on heterogeneous isotropic and anisotropic media using a family of analytical solutions with 4 intersecting fractures. Accounting for applications to multi-phase flow, we present in the appendix a Finite Volume formulation involving conservative fluxes, which applies for both schemes.

\section{Hybrid dimensional Darcy Flow Model in Fractured Porous Media}

\subsection{Geometry and Function Spaces}

Let $\Omega$ denote an open bounded domain of $\mathbb{R}^{d}, d=2,3$ assumed to be polyhedral for $d=3$ and polygonal for $d=2$. To fix ideas the dimension will be fixed to $d=3$ when it needs to be specified, for instance in the naming of the geometrical objects or for the space discretization in the next section. The adaptations to the case $d=2$ are straightforward. Let $\bar{\Gamma}=\bigcup_{i \in I} \bar{\Gamma}_{i}$ and its interior $\Gamma=\bar{\Gamma} \backslash \partial \bar{\Gamma}$ denote the network of fractures $\Gamma_{i} \subset \Omega, i \in I$, such that each $\Gamma_{i}$ is a planar polygonal simply connected open domain included in a plane $\mathcal{P}_{i}$ of $\mathbb{R}^{d}$. It is assumed that the angles of $\Gamma_{i}$ are strictly smaller than $2 \pi$, and that $\Gamma_{i} \cap \bar{\Gamma}_{j}=\emptyset$ for all $i \neq j$ - The assumption of planar fractures allows us to deal with conforming discretizations w.r.t. the fracture network. The other two assumptions are convenient, but not restrictive in practice.

For all $i \in I$, let us set $\Sigma_{i}=\partial \Gamma_{i}$, with $\mathbf{n}_{\Sigma_{i}}$ as unit vector in $\mathcal{P}_{i}$, normal to $\Sigma_{i}$ and outward to $\Gamma_{i}$. Further $\Sigma_{i, j}=\Sigma_{i} \cap \Sigma_{j}, j \in I \backslash\{i\}, \Sigma_{i, 0}=\Sigma_{i} \cap \partial \Omega, \Sigma_{i, N}=\Sigma_{i} \backslash\left(\bigcup_{j \in I \backslash\{i\}} \Sigma_{i, j} \cup \Sigma_{i, 0}\right)$, $\Sigma=\bigcup_{(i, j) \in I \times I, i \neq j}\left(\Sigma_{i, j} \backslash \Sigma_{i, 0}\right)$ and $\Sigma_{0}=\bigcup_{i \in I} \Sigma_{i, 0}$. It is assumed that $\Sigma_{i, 0}=\bar{\Gamma}_{i} \cap \partial \Omega$.

We will denote by $\mathrm{d} \tau(\mathbf{x})$ the $d-1$ dimensional Lebesgue measure on $\Gamma$. On the fracture 

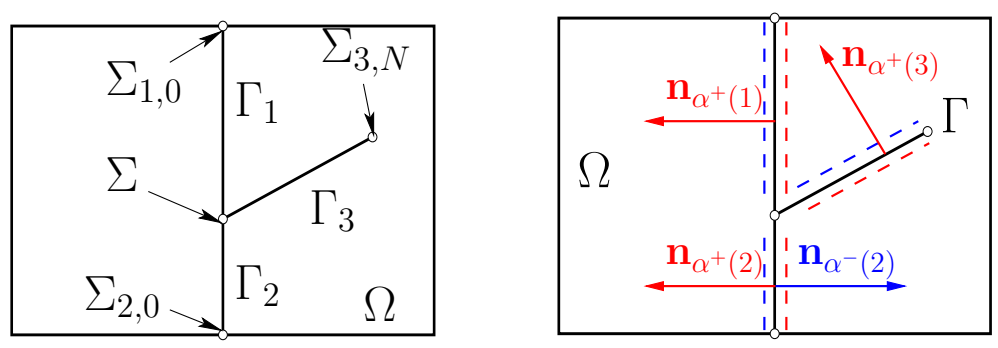

Figure 1: Example of a 2D domain $\Omega$ and 3 intersecting fractures $\Gamma_{i}, i=1,2,3$. We define the fracture plane orientations by $\alpha^{ \pm}(i) \in \chi$ for $\Gamma_{i}, i \in I$.

network $\Gamma$, we define the function space $L^{2}(\Gamma)=\left\{v=\left(v_{i}\right)_{i \in I}, v_{i} \in L^{2}\left(\Gamma_{i}\right), i \in I\right\}$, endowed with the norm $\|v\|_{L^{2}(\Gamma)}=\left(\sum_{i \in I}\left\|v_{i}\right\|_{L^{2}\left(\Gamma_{i}\right)}^{2}\right)^{\frac{1}{2}}$ and its subspace $H^{1}(\Gamma)$ consisting of functions $v=\left(v_{i}\right)_{i \in I}$ such that $v_{i} \in H^{1}\left(\Gamma_{i}\right), i \in I$ with continuous traces at the fracture intersections $\Sigma_{i, j}, j \in I \backslash\{i\}$. The space $H^{1}(\Gamma)$ is endowed with the norm $\|v\|_{H^{1}(\Gamma)}=\left(\sum_{i \in I}\left\|v_{i}\right\|_{H^{1}\left(\Gamma_{i}\right)}^{2}\right)^{\frac{1}{2}}$. We also define it's subspace with vanishing traces on $\Sigma_{0}$, which we denote by $H_{\Sigma_{0}}^{1}(\Gamma)$.

On $\Omega \backslash \bar{\Gamma}$, the gradient operator from $H^{1}(\Omega \backslash \bar{\Gamma})$ to $L^{2}(\Omega)^{d}$ is denoted by $\nabla$. On the fracture network $\Gamma$, the tangential gradient, acting from $H^{1}(\Gamma)$ to $L^{2}(\Gamma)^{d-1}$, is denoted by $\nabla_{\tau}$, and such that

$$
\nabla_{\tau} v=\left(\nabla_{\tau_{i}} v_{i}\right)_{i \in I}
$$

where, for each $i \in I$, the tangential gradient $\nabla_{\tau_{i}}$ is defined from $H^{1}\left(\Gamma_{i}\right)$ to $L^{2}\left(\Gamma_{i}\right)^{d-1}$ by fixing a reference Cartesian coordinate system of the plane $\mathcal{P}_{i}$ containing $\Gamma_{i}$. We also denote by $\operatorname{div}_{\tau_{i}}$ the divergence operator from $H_{\text {div }}\left(\Gamma_{i}\right)$ to $L^{2}\left(\Gamma_{i}\right)$.

We define the two unit normal vectors $n_{\alpha^{ \pm}(i)}$ at each planar fracture $\Gamma_{i}$, such that $\mathbf{n}_{\alpha^{+}(i)}+$ $\mathbf{n}_{\alpha^{-}(i)}=0$ (cf. figure 1). We define the set of indices $\chi=\left\{\alpha^{+}(i), \alpha^{-}(i) \mid i \in I\right\}$, such that $\# \chi=2 \# I$. For ease of notation, we use the convention $\Gamma_{\alpha^{+}(i)}=\Gamma_{\alpha^{+}(i)}=\Gamma_{i}$.

Then, for $\alpha=\alpha^{ \pm}(i) \in \chi$, we can define the trace operator on $\Gamma_{\alpha}$ :

$$
\gamma_{\alpha}: H^{1}(\Omega \backslash \bar{\Gamma}) \rightarrow L^{2}\left(\Gamma_{\alpha}\right)
$$

and the normal trace operator on $\Gamma_{\alpha}$ outward to the side $\alpha$ :

$$
\gamma_{\mathbf{n}, \alpha}: H_{\operatorname{div}}(\Omega \backslash \bar{\Gamma}) \rightarrow \mathcal{D}^{\prime}\left(\Gamma_{\alpha}\right)
$$

that satisfy $\gamma_{\alpha}(h)=\gamma_{\alpha}\left(h \uparrow_{\omega_{\alpha}}\right)$ and $\gamma_{\mathbf{n}, \alpha}(\mathbf{p})=\gamma_{\mathbf{n}, \alpha}\left(\mathbf{p} \uparrow_{\omega_{\alpha}}\right)$, where $\omega_{\alpha}=\left\{\mathbf{x} \in \Omega \mid(\mathbf{x}-\mathbf{y}) \cdot \mathbf{n}_{\alpha}<\right.$ $\left.0, \forall \mathbf{y} \in \Gamma_{i}\right\}$. Subsequently, the (one sided) jump operator on $\Gamma_{\alpha}$,

$$
\llbracket \cdot \rrbracket^{\alpha}: H^{1}(\Omega \backslash \bar{\Gamma}) \times H^{1}(\Gamma) \rightarrow L^{2}\left(\Gamma_{\alpha}\right)
$$

is defined by $\llbracket\left(h_{m}, h_{f}\right) \rrbracket^{\alpha}=\gamma_{\alpha} h_{m}-h_{f}$.

We now define the hybrid dimensional function spaces that will be used as variational spaces for the Darcy flow model in the next subsection, namely

$$
V=H^{1}(\Omega \backslash \bar{\Gamma}) \times H^{1}(\Gamma)
$$

and its subspace

$$
V^{0}=H_{\partial \Omega}^{1}(\Omega \backslash \bar{\Gamma}) \times H_{\Sigma_{0}}^{1}(\Gamma),
$$


where (with $\gamma_{\partial \Omega}: H^{1}(\Omega \backslash \bar{\Gamma}) \rightarrow L^{2}(\partial \Omega)$ denoting the trace operator on $\partial \Omega$ )

$$
H_{\partial \Omega}^{1}(\Omega \backslash \bar{\Gamma})=\left\{v \in H^{1}(\Omega \backslash \bar{\Gamma}) \mid \gamma_{\partial \Omega} v=0 \text { on } \partial \Omega\right\},
$$

as well as

$$
W=W_{m} \times W_{f}
$$

where

$$
\begin{aligned}
W_{m} & =\left\{\mathbf{q}_{m} \in H_{\operatorname{div}}(\Omega \backslash \bar{\Gamma}) \mid \gamma_{\mathbf{n}, \alpha} \mathbf{q}_{m} \in L^{2}\left(\Gamma_{\alpha}\right) \text { for all } \alpha \in \chi\right\} \text { and } \\
W_{f} & =\left\{\mathbf{q}_{f}=\left(\mathbf{q}_{f, i}\right)_{i \in I} \mid \mathbf{q}_{f, i} \in H_{\operatorname{div}}\left(\Gamma_{i}\right) \text { for all } i \in I\right. \\
& \text { and } \left.\sum_{i \in I} \int_{\Gamma_{i}}\left(\nabla_{\tau} v \cdot \mathbf{q}_{f, i}+v \cdot \operatorname{div}_{\tau_{i}} \mathbf{q}_{f, i}\right) \mathrm{d} \tau(\mathbf{x})=0 \text { for all } v \in H_{\Sigma_{0}}^{1}(\Gamma)\right\} .
\end{aligned}
$$

On $V$, we define the positive semidefinite, symmetric bilinear form

$$
\left(\left(u_{m}, u_{f}\right),\left(v_{m}, v_{f}\right)\right)_{V}=\int_{\Omega} \nabla u_{m} \cdot \nabla v_{m} \mathrm{~d} \mathbf{x}+\int_{\Gamma} \nabla_{\tau} u_{f} \cdot \nabla_{\tau} v_{f} \mathrm{~d} \tau(\mathbf{x})+\sum_{\alpha \in \chi} \int_{\Gamma_{\alpha}} \llbracket u \rrbracket^{\alpha} \llbracket v \rrbracket^{\alpha} \mathrm{d} \tau(\mathbf{x}),
$$

for $u=\left(u_{m}, u_{f}\right), v=\left(v_{m}, v_{f}\right) \in V$. Note that $(\cdot, \cdot)_{V}$ is a scalar product on $V^{0}$ with induced norm denoted by $\|\cdot\|_{V^{0}}$ in the following. We define for all $\left(\mathbf{p}_{m}, \mathbf{p}_{f}\right),\left(\mathbf{q}_{m}, \mathbf{q}_{f}\right) \in W$ the scalar product

$$
\begin{aligned}
\left(\left(\mathbf{p}_{m}, \mathbf{p}_{f}\right),\left(\mathbf{q}_{m}, \mathbf{q}_{f}\right)\right)_{W} & =\int_{\Omega} \mathbf{p}_{m} \mathbf{q}_{m} \mathrm{~d} \mathbf{x}+\int_{\Omega} \operatorname{div} \mathbf{p}_{m} \cdot \operatorname{div} \mathbf{q}_{m} \mathrm{~d} \mathbf{x} \\
& +\int_{\Gamma} \mathbf{p}_{f} \mathbf{q}_{f} \mathrm{~d} \tau(\mathbf{x})+\int_{\Gamma} \operatorname{div}_{\tau} \mathbf{p}_{f} \cdot \operatorname{div}_{\tau} \mathbf{q}_{f} \mathrm{~d} \tau(\mathbf{x}) \\
& +\sum_{\alpha \in \chi} \int_{\Gamma_{\alpha}}\left(\gamma_{\mathbf{n}, \alpha} \mathbf{p}_{m} \cdot \gamma_{\mathbf{n}, \alpha} \mathbf{q}_{m}\right) d \tau(\mathbf{x}),
\end{aligned}
$$

which induces the norm $\left\|\left(\mathbf{q}_{m}, \mathbf{q}_{f}\right)\right\|_{W}$, and where we have used the notation $\operatorname{div}_{\tau} \mathbf{p}_{f}=\operatorname{div}_{\tau_{i}} \mathbf{p}_{f, i}$ on $\Gamma_{i}$ for all $i \in I$ and $\mathbf{p}_{f}=\left(\mathbf{p}_{f, i}\right)_{i \in I} \in W_{f}$.

Using similar arguments as in the proof of [22], example II.3.4, one can prove the following Poincaré type inequality.

Proposition 2.1 The norm $\|\cdot\|_{V^{0}}$ satisfies the following inequality

$$
\left\|v_{m}\right\|_{H^{1}(\Omega \backslash \bar{\Gamma})}+\left\|v_{f}\right\|_{H^{1}(\Gamma)} \leq \mathcal{C}_{P}\left\|\left(v_{m}, v_{f}\right)\right\|_{V^{0}}
$$

for all $\left(v_{m}, v_{f}\right) \in V^{0}$.

The convergence analysis presented in section 4 requires some results on the density of smooth subspaces of $V$ and $W$, which we state below.

Definition 2.1 $1 . C_{\Omega}^{\infty}$ is defined as the subspace of functions in $C_{b}^{\infty}(\Omega \backslash \bar{\Gamma})$ vanishing on a neighbourhood of the boundary $\partial \Omega$, where $C_{b}^{\infty}(\Omega \backslash \bar{\Gamma}) \subset C^{\infty}(\Omega \backslash \bar{\Gamma})$ is the set of functions $\varphi$, such that for all $\mathrm{x} \in \Omega$ there exists $r>0$, such that for all connected components $\omega$ of $\left\{\mathbf{x}+\mathbf{y} \in \mathbb{R}^{d}|| \mathbf{y} \mid<r\right\} \cap(\Omega \backslash \bar{\Gamma})$ one has $\varphi \in C^{\infty}(\bar{\omega})$.

2. $C_{\Gamma}^{\infty}=\gamma_{\Gamma}\left(C_{0}^{\infty}(\Omega)\right)$ is defined as the image of $C_{0}^{\infty}(\Omega)$ of the trace operator $\gamma_{\Gamma}: H_{0}^{1}(\Omega) \rightarrow$ $L^{2}(\Gamma)$. 


$$
\begin{aligned}
& \text { 3. } C_{W_{m}}^{\infty}=C_{b}^{\infty}(\Omega \backslash \bar{\Gamma})^{d} \text {. } \\
& \text { 4. } C_{W_{f}}^{\infty}=\left\{\mathbf{q}_{f}=\left(\mathbf{q}_{f, i}\right)_{i \in I} \mid \mathbf{q}_{f, i} \in C^{\infty}\left(\bar{\Gamma}_{i}\right)^{d-1}, \sum_{i \in I} \mathbf{q}_{f, i} \cdot \mathbf{n}_{\Sigma_{i}}=0 \text { on } \Sigma, \mathbf{q}_{f, i} \cdot \mathbf{n}_{\Sigma_{i}}=\right. \\
& \left.0 \text { on } \Sigma_{i, N}, i \in I\right\} .
\end{aligned}
$$

The proofs of the two following propositions can be found in the appendix.

Proposition $2.2 C_{\Omega}^{\infty} \times C_{\Gamma}^{\infty}$ is dense in $V^{0}$.

Proposition 2.3 $C_{W_{m}}^{\infty} \times C_{W_{f}}^{\infty}$ is dense in $W$.

\subsection{Darcy Flow Model}

In the matrix domain $\Omega \backslash \bar{\Gamma}$, let us denote by $\Lambda_{m} \in L^{\infty}(\Omega)^{d \times d}$ the permeability tensor such that there exist $\bar{\lambda}_{m} \geq \underline{\lambda}_{m}>0$ with

$$
\underline{\lambda}_{m}|\zeta|^{2} \leq\left(\Lambda_{m}(\mathbf{x}) \zeta, \zeta\right) \leq \bar{\lambda}_{m}|\zeta|^{2} \text { for all } \zeta \in \mathbb{R}^{d}, \mathbf{x} \in \Omega
$$

Analogously, in the fracture network $\Gamma$, we denote by $\Lambda_{f} \in L^{\infty}(\Gamma)^{(d-1) \times(d-1)}$ the tangential permeability tensor, and assume that there exist $\bar{\lambda}_{f} \geq \underline{\lambda}_{f}>0$, such that holds

$$
\underline{\lambda}_{f}|\zeta|^{2} \leq\left(\Lambda_{f}(\mathbf{x}) \zeta, \zeta\right) \leq \bar{\lambda}_{f}|\zeta|^{2} \text { for all } \zeta \in \mathbb{R}^{d-1}, \mathbf{x} \in \Gamma \text {. }
$$

At the fracture network $\Gamma$, we introduce the orthonormal system $\left(\boldsymbol{\tau}_{1}(\mathbf{x}), \boldsymbol{\tau}_{2}(\mathbf{x}), \mathbf{n}(\mathbf{x})\right)$, defined a.e. on $\Gamma$. Inside the fractures, the normal direction is assumed to be a permeability principal direction. The normal permeability $\lambda_{f, \mathbf{n}} \in L^{\infty}(\Gamma)$ is such that $\underline{\lambda}_{f, \mathbf{n}} \leq \lambda_{f, \mathbf{n}}(\mathbf{x}) \leq \bar{\lambda}_{f, \mathbf{n}}$ for a.e. $\mathbf{x} \in \Gamma$ with $0<\underline{\lambda}_{f, \mathbf{n}} \leq \bar{\lambda}_{f, \mathbf{n}}$. We also denote by $d_{f} \in L^{\infty}(\Gamma)$ the width of the fractures assumed to be such that there exist $\bar{d}_{f} \geq \underline{d}_{f}>0$ with, for a.e. $\mathbf{x} \in \Gamma$,

$$
\underline{d}_{f} \leq d_{f}(\mathbf{x}) \leq \bar{d}_{f},
$$

Let us define the weighted Lebesgue $d-1$ dimensional measure on $\Gamma$ by $d \tau_{f}(\mathbf{x})=d_{f}(\mathbf{x}) \mathrm{d} \tau(\mathbf{x})$. We consider the source terms $h_{m} \in L^{2}(\Omega)$ (resp. $h_{f} \in L^{2}(\Gamma)$ ) in the matrix domain $\Omega \backslash \bar{\Gamma}$ (resp. in the fracture network $\Gamma$ ). The half normal transmissibility in the fracture network is denoted by $T_{f}=\frac{2 \lambda_{f, \mathbf{n}}}{d_{f}}$.

\subsubsection{Strong formulation}

Given $\xi \in\left(\frac{1}{2}, 1\right]$, the PDEs model writes: find $u=\left(u_{m}, u_{f}\right) \in V^{0}, \mathbf{q}=\left(\mathbf{q}_{m}, \mathbf{q}_{f}\right) \in W$ such that:

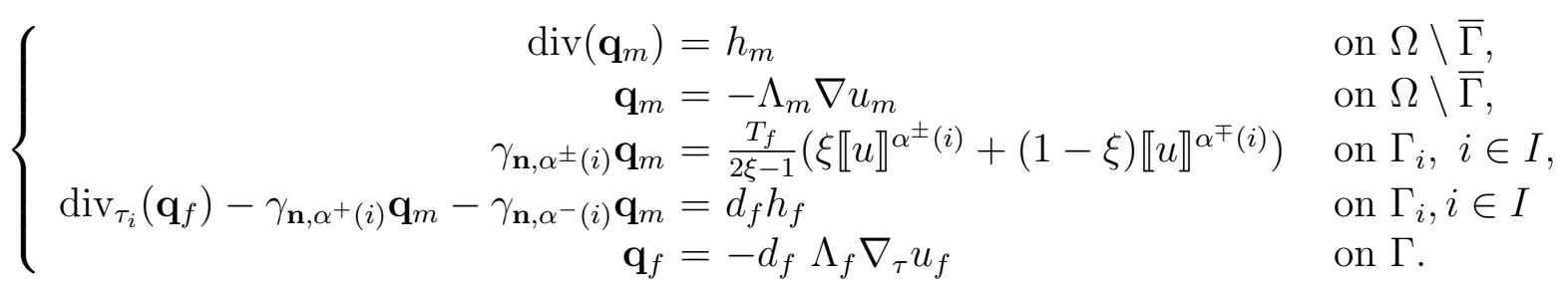

The boundary conditions of the model are contained in the definitions of the function spaces $V^{0}, W$, which are stated in Subsection 2.1. 


\subsubsection{Weak formulation}

The hybrid dimensional weak formulation amounts to find $u=\left(u_{m}, u_{f}\right) \in V^{0}$ satisfying the following variational equality for all $v=\left(v_{m}, v_{f}\right) \in V^{0}$ :

$$
\begin{aligned}
& \int_{\Omega} \Lambda_{m} \nabla u_{m} \cdot \nabla v_{m} \mathrm{~d} \mathbf{x}+\int_{\Gamma} \Lambda_{f} \nabla_{\tau} u_{f} \cdot \nabla_{\tau} v_{f} \mathrm{~d} \tau_{f}(\mathbf{x}) \\
& +\sum_{i \in I} \int_{\Gamma_{i}} \frac{T_{f}}{2 \xi-1} \sum_{(\alpha, \beta) \in\left\{\left(\alpha^{ \pm}(i), \alpha \mp(i)\right)\right\}}\left(\xi \llbracket u \rrbracket^{\alpha}+(1-\xi) \llbracket u \rrbracket^{\beta}\right) \llbracket v \rrbracket^{\alpha} \mathrm{d} \tau(\mathbf{x}) \\
& -\int_{\Omega} h_{m} v_{m} \mathrm{~d} \mathbf{x}-\int_{\Gamma} h_{f} v_{f} \mathrm{~d} \tau_{f}(\mathbf{x})=0 .
\end{aligned}
$$

The following proposition states the well posedness of the variational formulation (2.2).

Proposition 2.4 For all $\xi \in\left(\frac{1}{2}, 1\right]$, the variational problem $(2.2)$ has a unique solution $\left(u_{m}, u_{f}\right) \in$ $V^{0}$ which satisfies the a priori estimate

$$
\left\|\left(u_{m}, u_{f}\right)\right\|_{V^{0}} \leq C\left(\left\|h_{m}\right\|_{L^{2}(\Omega)}+\left\|h_{f}\right\|_{L^{2}(\Gamma)}\right),
$$

with $C$ depending only on $\xi, \mathcal{C}_{P}, \underline{\lambda}_{m}, \underline{\lambda}_{f}, \underline{d}_{f}, \bar{d}_{f}$, and $\underline{\lambda}_{f, n}$. In addition $\left(\mathbf{q}_{m}, \mathbf{q}_{f}\right)=-\left(\Lambda_{m} \nabla u_{m}, d_{f} \Lambda_{f} \nabla_{\tau} u_{f}\right)$ belongs to $W$.

Proof Using that for all $\xi \in\left(\frac{1}{2}, 1\right]$ and for all $(a, b) \in \mathbb{R}^{2}$ one has

$$
a^{2}+b^{2} \leq(\xi a+(1-\xi) b) a+(\xi b+(1-\xi) a) b \leq \frac{1}{2 \xi-1}\left(a^{2}+b^{2}\right),
$$

the Lax-Milgram Theorem applies, which ensures the statement of the proposition.

\section{Gradient Discretization of the Hybrid Dimensional Model}

\subsection{Gradient Scheme Framework}

A gradient discretization $\mathcal{D}$ of hybrid dimensional Darcy flow models is defined by a vector space of degrees of freedom $X_{\mathcal{D}}=X_{\mathcal{D}_{m}} \times X_{\mathcal{D}_{f}}$, its subspace satisfying ad hoc homogeneous boundary conditions $X_{\mathcal{D}}^{0}=X_{\mathcal{D}_{m}}^{0} \times X_{\mathcal{D}_{f}}^{0}$, and the following gradient and reconstruction operators:

- Gradient operator on the matrix domain: $\nabla_{\mathcal{D}_{m}}: X_{\mathcal{D}_{m}} \rightarrow L^{2}(\Omega)^{d}$

- Gradient operator on the fracture network: $\nabla_{\mathcal{D}_{f}}: X_{\mathcal{D}_{f}} \rightarrow L^{2}(\Gamma)^{d-1}$

- A function reconstruction operator on the matrix domain: $\Pi_{\mathcal{D}_{m}}: X_{\mathcal{D}_{m}} \rightarrow L^{2}(\Omega)$

- A function reconstruction operator on the fracture network: $\Pi_{\mathcal{D}_{f}}: X_{\mathcal{D}_{f}} \rightarrow L^{2}(\Gamma)$

- Reconstruction operators of the jump on $\Gamma_{\alpha}$ for $\alpha \in \chi$ : $\llbracket \cdot \rrbracket_{\mathcal{D}}^{\alpha}: X_{\mathcal{D}} \rightarrow L^{2}\left(\Gamma_{\alpha}\right)$. 
The space $X_{\mathcal{D}}$ is endowed with the seminorm

$$
\left\|\left(v_{\mathcal{D}_{m}}, v_{\mathcal{D}_{f}}\right)\right\|_{\mathcal{D}}=\left(\left\|\nabla_{\mathcal{D}_{m}} v_{\mathcal{D}_{m}}\right\|_{L^{2}(\Omega)^{d}}^{2}+\left\|\nabla_{\mathcal{D}_{f}} v_{\mathcal{D}_{f}}\right\|_{L^{2}(\Gamma)^{d-1}}^{2}+\sum_{\alpha \in \chi}\left\|\llbracket v_{\mathcal{D}} \rrbracket_{\mathcal{D}}^{\alpha}\right\|_{L^{2}\left(\Gamma_{\alpha}\right)}^{2}\right)^{\frac{1}{2}},
$$

which is assumed to define a norm on $X_{\mathcal{D}}^{0}$.

Discretizing (2.2) in the sense of gradient discretizations yields: find $u_{\mathcal{D}}=\left(u_{\mathcal{D}_{m}}, u_{\mathcal{D}_{f}}\right) \in X_{\mathcal{D}}^{0}$ such that

$$
\begin{aligned}
& \int_{\Omega} \Lambda_{m} \nabla_{\mathcal{D}_{m}} u_{\mathcal{D}_{m}} \cdot \nabla_{\mathcal{D}_{m}} v_{\mathcal{D}_{m}} \mathrm{~d} \mathbf{x}+\int_{\Gamma} \Lambda_{f} \nabla_{\mathcal{D}_{f}} u_{\mathcal{D}_{f}} \cdot \nabla_{\mathcal{D}_{f}} v_{\mathcal{D}_{f}} \mathrm{~d} \tau_{f}(\mathbf{x})+\sum_{i \in I} \int_{\Gamma_{i}} \frac{T_{f}}{2 \xi-1} \\
& \sum_{(\alpha, \beta) \in\left\{\left(\alpha^{ \pm}(i), \alpha^{\mp}(i)\right)\right\}}\left(\xi \llbracket u_{\mathcal{D}} \rrbracket_{\mathcal{D}}^{\alpha}+(1-\xi) \llbracket u_{\mathcal{D}} \rrbracket_{\mathcal{D}}^{\beta}\right) \llbracket v_{\mathcal{D}} \rrbracket_{\mathcal{D}}^{\alpha} \mathrm{d} \tau(\mathbf{x}) \\
& -\int_{\Omega} h_{m} \Pi_{\mathcal{D}_{m}} v_{\mathcal{D}_{m}} \mathrm{~d} \mathbf{x}-\int_{\Gamma} h_{f} \Pi_{\mathcal{D}_{f}} v_{\mathcal{D}_{f}} \mathrm{~d} \tau_{f}(\mathbf{x})=0,
\end{aligned}
$$

for all $v_{\mathcal{D}}=\left(v_{\mathcal{D}_{m}}, v_{\mathcal{D}_{f}}\right) \in X_{\mathcal{D}}^{0}$.

Of course the above definition of a gradient discretization is at this stage very general. Some stability and consistency conditions must be imposed in order to expect a well posed and convergent discretization. Since the gradient scheme framework accounts for non conforming discretizations (in the sense that $\nabla_{\mathcal{D}_{m}} \neq \nabla \Pi_{\mathcal{D}_{m}}$ or or $\nabla_{\mathcal{D}_{f}} \neq \nabla_{\tau} \Pi_{\mathcal{D}_{f}}$ or $\llbracket \cdot \rrbracket_{\mathcal{D}}^{\alpha} \neq \llbracket \Pi_{\mathcal{D}_{m}}, \Pi_{\mathcal{D}_{f}} \rrbracket^{\alpha}$ ), a third condition must be assumed, which roughly speaking states that integration by part w.r.t. the discrete operators provides a residual "close" to zero.

Coercivity: Let

$$
\operatorname{C}_{\mathcal{D}}=\max _{0 \neq\left(v_{\mathcal{D}_{m}}, v_{\mathcal{D}_{f}}\right) \in X_{\mathcal{D}}^{0}} \frac{\left\|\Pi_{\mathcal{D}_{m}} v_{\mathcal{D}_{m}}\right\|_{L^{2}(\Omega)}+\left\|\Pi_{\mathcal{D}_{f}} v_{\mathcal{D}_{f}}\right\|_{L^{2}(\Gamma)}}{\left\|\left(v_{\mathcal{D}_{m}}, v_{\mathcal{D}_{f}}\right)\right\|_{\mathcal{D}}} .
$$

A sequence $\left(\mathcal{D}^{l}\right)_{l \in \mathbb{N}}$ of gradient discretizations is said to be coercive, if there exists $\overline{\mathcal{C}}_{P}>0$ such that $\mathcal{C}_{\mathcal{D}^{l}} \leq \overline{\mathcal{C}}_{P}$ for all $l \in \mathbb{N}$.

Consistency: For $u=\left(u_{m}, u_{f}\right) \in V^{0}$ and $v_{\mathcal{D}}=\left(v_{\mathcal{D}_{m}}, v_{\mathcal{D}_{f}}\right) \in X_{\mathcal{D}}^{0}$ let us define

$$
\begin{aligned}
s\left(v_{\mathcal{D}}, u\right) & =\left\|\nabla_{\mathcal{D}_{m}} v_{\mathcal{D}_{m}}-\nabla u_{m}\right\|_{L^{2}(\Omega)}+\left\|\nabla_{\mathcal{D}_{f}} v_{\mathcal{D}_{f}}-\nabla_{\tau} u_{f}\right\|_{L^{2}(\Gamma)^{d-1}} \\
& +\left\|\Pi_{\mathcal{D}_{m}} v_{\mathcal{D}_{m}}-u_{m}\right\|_{L^{2}(\Omega)}+\left\|\Pi_{\mathcal{D}_{f}} v_{\mathcal{D}_{f}}-u_{f}\right\|_{L^{2}(\Gamma)}+\sum_{\alpha \in \chi}\left\|\llbracket v_{\mathcal{D}} \rrbracket_{\mathcal{D}}^{\alpha}-\llbracket u \rrbracket^{\alpha}\right\|_{L^{2}\left(\Gamma_{\alpha}\right)}
\end{aligned}
$$

and $\mathcal{S}_{\mathcal{D}}(u)=\min _{v_{\mathcal{D}} \in X_{\mathcal{D}}^{0}} s\left(v_{\mathcal{D}}, u\right)$. A sequence $\left(\mathcal{D}^{l}\right)_{l \in \mathbb{N}}$ of gradient discretizations is said to be consistent, if for all $u=\left(u_{m}, u_{f}\right) \in V^{0}$ holds

$$
\lim _{l \rightarrow \infty} \mathcal{S}_{\mathcal{D}^{l}}(u)=0
$$

Limit Conformity: For all $\mathbf{q}=\left(\mathbf{q}_{m}, \mathbf{q}_{f}\right) \in W, v_{\mathcal{D}}=\left(v_{\mathcal{D}_{m}}, v_{\mathcal{D}_{f}}\right)$ we define

$$
\begin{aligned}
w\left(v_{\mathcal{D}}, \mathbf{q}\right) & =\int_{\Omega}\left(\nabla_{\mathcal{D}_{m}} v_{\mathcal{D}_{m}} \cdot \mathbf{q}_{m}+\left(\Pi_{\mathcal{D}_{m}} v_{\mathcal{D}_{m}}\right) \operatorname{div} \mathbf{q}_{m}\right) \mathrm{d} \mathbf{x} \\
& +\int_{\Gamma}\left(\nabla_{\mathcal{D}_{f}} v_{\mathcal{D}_{f}} \cdot \mathbf{q}_{f}+\left(\Pi_{\mathcal{D}_{f}} v_{\mathcal{D}_{f}}\right) \operatorname{div}_{\tau} \mathbf{q}_{f}\right) \mathrm{d} \tau(\mathbf{x}) \\
& -\sum_{\alpha \in \chi} \int_{\Gamma_{\alpha}} \gamma_{\mathbf{n}, \alpha} \mathbf{q}_{m}\left(\llbracket v_{\mathcal{D}} \rrbracket_{\mathcal{D}}^{\alpha}+\Pi_{\mathcal{D}_{f}} v_{\mathcal{D}_{f}}\right) \mathrm{d} \tau(\mathbf{x})
\end{aligned}
$$


and $\mathcal{W}_{\mathcal{D}}(\mathbf{q})=\max _{0 \neq v_{\mathcal{D}} \in X_{\mathcal{D}}^{0}} \frac{1}{\left\|v_{\mathcal{D}}\right\|_{\mathcal{D}}}\left|w\left(v_{\mathcal{D}}, \mathbf{q}\right)\right|$. A sequence $\left(\mathcal{D}^{l}\right)_{l \in \mathbb{N}}$ of gradient discretizations is said to be limit conforming, if for all $\mathbf{q}=\left(\mathbf{q}_{m}, \mathbf{q}_{f}\right) \in W$ holds

$$
\lim _{l \rightarrow \infty} \mathcal{W}_{\mathcal{D}^{l}}(\mathbf{q})=0
$$

As will be seen in the two examples of gradient schemes in the next section, the practical definition of the operators $\Pi_{\mathcal{D}_{m}}$ and $\Pi_{\mathcal{D}_{f}}$ satisfying the above assumptions is very flexible. This flexibility can be exploited in order to adapt the discretization to heterogeneities when coupling the Darcy flow model with a transport equation (see [16] for details). On the other hand, the choice of the operators $\llbracket \cdot \rrbracket_{\mathcal{D}}^{\alpha}$ is more constrained by the limit conformity property.

The limit conformity property is enhanced by the following proposition which roughly speaking states that, the limit(s) of bounded sequences in $X_{\mathcal{D}^{l}}^{0}, l \in \mathbb{N}$, belong to $V^{0}$.

Proposition 3.1 (Regularity at the Limit) Let $\left(\mathcal{D}^{l}\right)_{l \in \mathbb{N}}$ be a coercive and limit conforming sequence of gradient discretizations and let $v_{\mathcal{D}^{l}}=\left(v_{\mathcal{D}_{m}^{l}}, v_{\mathcal{D}_{f}^{l}}\right)_{l \in \mathbb{N}}$ be a uniformly bounded sequence in $X_{\mathcal{D}^{l}}^{0}$. Then, there exist $v=\left(v_{m}, v_{f}\right) \in V^{0}$ and a subsequence still denoted by $\left(v_{\mathcal{D}^{l}}\right)_{l \in \mathbb{N}}$ such that

$$
\left\{\begin{array}{l}
\Pi_{\mathcal{D}_{m}^{l}} v_{\mathcal{D}_{m}^{l}} \rightarrow v_{m} \quad \text { in } L^{2}(\Omega), \\
\nabla_{\mathcal{D}_{m}^{l}} v_{\mathcal{D}_{m}^{l}} \rightarrow \nabla v_{m} \quad \text { in } L^{2}(\Omega)^{d}, \\
\Pi_{\mathcal{D}_{f}^{l}} v_{\mathcal{D}_{f}^{l}} \rightarrow v_{f} \quad \text { in } L^{2}(\Gamma), \\
\nabla_{\mathcal{D}_{f}^{l}} v_{\mathcal{D}_{f}^{l}} \rightarrow \nabla_{\tau} v_{f} \quad \text { in } L^{2}(\Gamma)^{d-1}, \\
\llbracket v_{\mathcal{D}^{l}} \rrbracket_{\mathcal{D}^{l}}^{\alpha} \rightarrow \llbracket v \rrbracket^{\alpha} \quad \text { in } L^{2}\left(\Gamma_{\alpha}\right), \text { for all } \alpha \in \chi .
\end{array}\right.
$$

Proof By definition of the norm of $X_{\mathcal{D}^{l}}^{0}$ and by coercivity, $\Pi_{\mathcal{D}_{m}^{l}} v_{\mathcal{D}_{m}^{l}}, \Pi_{\mathcal{D}_{f}^{l}} v_{\mathcal{D}_{f}^{l}}, \nabla_{\mathcal{D}_{m}} v_{\mathcal{D}_{m}^{l}}, \nabla_{\mathcal{D}_{f}} v_{\mathcal{D}_{f}^{l}}$ and $\left(\llbracket u_{\mathcal{D}^{l}} \rrbracket_{\mathcal{D}^{l}}^{\alpha}\right), \quad \alpha \in \chi$, are uniformly bounded in $L^{2}$ (for $\left.l \rightarrow \infty\right)$. Therefore there exist $v_{m} \in L^{2}(\Omega), v_{f} \in L^{2}(\Gamma), G \in L^{2}(\Omega)^{d}, H \in L^{2}(\Gamma)^{d-1}$ and $J_{\alpha} \in L^{2}\left(\Gamma_{\alpha}\right), \alpha \in \chi$, and a subsequence still denoted by $\left(v_{\mathcal{D}_{m}^{l}}, v_{\mathcal{D}_{f}^{l}}\right)_{l \in \mathbb{N}}$ such that

$$
\begin{array}{ll}
\Pi_{\mathcal{D}_{m}^{l}} v_{\mathcal{D}_{m}^{l}} \rightarrow v_{m} & \text { in } L^{2}(\Omega), \\
\nabla_{\mathcal{D}_{m}} v_{\mathcal{D}_{m}^{l}} \rightarrow G & \text { in } L^{2}(\Omega)^{d}, \\
\Pi_{\mathcal{D}_{f}^{l}} v_{\mathcal{D}_{f}^{l}} \rightarrow v_{f} & \text { in } L^{2}(\Gamma), \\
\nabla_{\mathcal{D}_{f}} v_{\mathcal{D}_{f}^{l}} \rightarrow H & \text { in } L^{2}(\Gamma)^{d-1}, \\
\llbracket v_{\mathcal{D}^{l}} \rrbracket_{\mathcal{D}^{l}}^{\alpha} \rightarrow J_{\alpha} & \text { in } L^{2}\left(\Gamma_{\alpha}\right), \text { for } \alpha \in \chi .
\end{array}
$$

Using limit conformity we obtain (by letting $l \rightarrow \infty$ )

$$
\int_{\Omega}\left(G \cdot \mathbf{q}_{m}+v_{m} \operatorname{div} \mathbf{q}_{m}\right) \mathrm{d} \mathbf{x}+\int_{\Gamma}\left(H \cdot \mathbf{q}_{f}+v_{f} \operatorname{div}_{\tau} \mathbf{q}_{f}\right) \mathrm{d} \tau(\mathbf{x})-\sum_{\alpha \in \chi} \int_{\Gamma_{\alpha}} \gamma_{\mathbf{n}, \alpha} \mathbf{q}_{m}\left(J_{\alpha}+v_{f}\right) \mathrm{d} \tau(\mathbf{x})=0
$$

for all $\left(\mathbf{q}_{m}, \mathbf{q}_{f}\right) \in C_{W_{m}}^{\infty} \times C_{W_{f}}^{\infty}$. The statement of the proposition follows now from Lemma A.1.

Corollary 3.1 Let $\left(\mathcal{D}^{l}\right)_{l \in \mathbb{N}}$ be a sequence of gradient discretizations, assumed to be limit conforming against regular test functions $\left(\mathbf{q}_{m}, \mathbf{q}_{f}\right) \in C_{W_{m}}^{\infty} \times C_{W_{f}}^{\infty}$ and let $\left(v_{\mathcal{D}_{m}^{l}}, v_{\mathcal{D}_{f}^{l}}\right)_{l \in \mathbb{N}}$ be a uniformly bounded sequence in $X_{\mathcal{D}^{l}}^{0}$, such that $\Pi_{\mathcal{D}_{m}^{l}} v_{\mathcal{D}_{m}^{l}}$ and $\Pi_{\mathcal{D}_{f}^{l}} v_{\mathcal{D}_{f}^{l}}$ are uniformly bounded in $L^{2}$ $($ for $l \rightarrow \infty)$. Then holds the conclusion of Proposition 3.1. 


\subsection{Application to (2.2)}

We now want to exploit the previous results for the non conforming discrete variational formulation of the model problem, (3.1).

Proposition 3.2 Let $\xi \in\left(\frac{1}{2}, 1\right]$ and $\mathcal{D}$ be a gradient discretization, then (3.1) has a unique solution $\left(u_{\mathcal{D}_{m}}, u_{\mathcal{D}_{f}}\right) \in X_{\mathcal{D}}^{0}$ satisfying the a priori estimate

$$
\left\|\left(u_{\mathcal{D}_{m}}, u_{\mathcal{D}_{f}}\right)\right\|_{\mathcal{D}} \leq C\left(\left\|h_{m}\right\|_{L^{2}(\Omega)}+\left\|h_{f}\right\|_{L^{2}(\Gamma)}\right)
$$

with $C$ depending only on $\xi, \mathcal{C}_{\mathcal{D}}, \underline{\lambda}_{m}, \underline{\lambda}_{f}, \underline{d}_{f}, \bar{d}_{f}$, and $\underline{\lambda}_{f, n}$.

Proof The Lax-Milgram Theorem applies, which ensures this result.

The main theoretical result for gradient schemes is stated by the following proposition:

Proposition 3.3 (Error Estimate) Let $u=\left(u_{m}, u_{f}\right) \in V^{0}, \mathbf{q}=\left(\mathbf{q}_{m}, \mathbf{q}_{f}\right) \in W$ be the solution of (2.1). Let $\xi \in\left(\frac{1}{2}, 1\right], \mathcal{D}$ be a gradient discretization and $u_{\mathcal{D}}=\left(u_{\mathcal{D}_{m}}, u_{\mathcal{D}_{f}}\right) \in X_{\mathcal{D}}^{0}$ be the solution of (3.1). Then, there exists $C_{0}>0$ depending only on $\xi, \mathcal{C}_{\mathcal{D}}, \underline{\lambda}_{m}, \underline{\lambda}_{f}, \bar{\lambda}_{m}, \bar{\lambda}_{f}, \underline{d}_{f}, \bar{d}_{f}$, $\underline{\lambda}_{f, n}$, and $\bar{\lambda}_{f, n}$ such that one has the following error estimate:

$$
\begin{aligned}
& \left\|\Pi_{\mathcal{D}_{m}} u_{\mathcal{D}_{m}}-u_{m}\right\|_{L^{2}(\Omega)}+\left\|\Pi_{\mathcal{D}_{f}} u_{\mathcal{D}_{f}}-u_{f}\right\|_{L^{2}(\Gamma)}+\sum_{\alpha \in \chi}\left\|\llbracket u_{\mathcal{D}} \rrbracket_{\mathcal{D}}^{\alpha}-\llbracket u \rrbracket^{\alpha}\right\|_{L^{2}\left(\Gamma_{\alpha}\right)} \\
& +\left\|\nabla u_{m}-\nabla_{\mathcal{D}_{m}} u_{\mathcal{D}_{m}}\right\|_{L^{2}(\Omega)^{d}}+\left\|\nabla_{\tau} u_{f}-\nabla_{\mathcal{D}_{f}} u_{\mathcal{D}_{f}}\right\|_{L^{2}(\Gamma)^{d-1}} \leq C_{0}\left(\mathcal{S}_{\mathcal{D}}\left(u_{m}, u_{f}\right)+\mathcal{W}_{\mathcal{D}}\left(\mathbf{q}_{m}, \mathbf{q}_{f}\right)\right) .
\end{aligned}
$$

Proof From the definition of $\mathcal{W}_{\mathcal{D}}$, and using the definitions (2.1) of the solution $u, \mathbf{q}$ and (3.1) of the discrete solution $u_{\mathcal{D}}$, it holds for all $\left(v_{\mathcal{D}_{m}}, v_{\mathcal{D}_{f}}\right) \in X_{\mathcal{D}}^{0}$

$$
\begin{aligned}
& \left\|\left(v_{\mathcal{D}_{m}}, v_{\mathcal{D}_{f}}\right)\right\|_{\mathcal{D}} \cdot \mathcal{W}_{\mathcal{D}}\left(\mathbf{q}_{m}, \mathbf{q}_{f}\right) \\
& \geq \mid \int_{\Omega}\left(\nabla_{\mathcal{D}_{m}} v_{\mathcal{D}_{m}} \cdot \mathbf{q}_{m}+\left(\Pi_{\mathcal{D}_{m}} v_{\mathcal{D}_{m}}\right) h_{m}\right) \mathrm{d} \mathbf{x}+\int_{\Gamma}\left(\nabla_{\mathcal{D}_{f}} v_{\mathcal{D}_{f}} \cdot \mathbf{q}_{f}+\left(\Pi_{\mathcal{D}_{f}} v_{\mathcal{D}_{f}}\right) d_{f} h_{f}\right) \mathrm{d} \tau(\mathbf{x}) \\
& -\sum_{i \in I} \int_{\Gamma_{i}} \frac{T_{f}}{2 \xi-1} \sum_{(\alpha, \beta) \in\left\{\left(\alpha^{ \pm}(i), \alpha^{\mp}(i)\right)\right\}}\left(\xi \llbracket u \rrbracket^{\alpha}+(1-\xi) \llbracket u \rrbracket^{\beta}\right) \llbracket v_{\mathcal{D}} \rrbracket_{\mathcal{D}}^{\alpha} \mathrm{d} \tau(\mathbf{x}) \mid \\
& =\mid \int_{\Omega}\left(\Lambda_{m} \nabla_{\mathcal{D}_{m}} v_{\mathcal{D}_{m}} \cdot\left(\nabla_{\mathcal{D}_{m}} u_{\mathcal{D}_{m}}-\nabla u_{m}\right)\right) \mathrm{d} \mathbf{x}+\int_{\Gamma}\left(\Lambda_{f} \nabla_{\mathcal{D}_{f}} v_{\mathcal{D}_{f}} \cdot\left(\nabla_{\mathcal{D}_{f}} u_{\mathcal{D}_{f}}-\nabla_{\tau} u_{f}\right)\right) \mathrm{d} \tau_{f}(\mathbf{x}) \\
& -\sum_{i \in I} \int_{\Gamma_{i}} \frac{T_{f}}{2 \xi-1} \sum_{(\alpha, \beta) \in\left\{\left(\alpha^{ \pm}(i), \alpha \mp(i)\right)\right\}}\left(\xi \llbracket u \rrbracket^{\alpha}+(1-\xi) \llbracket u \rrbracket^{\beta}-\xi \llbracket u_{\mathcal{D}} \rrbracket_{\mathcal{D}}^{\alpha}-(1-\xi) \llbracket u_{\mathcal{D}} \rrbracket_{\mathcal{D}}^{\beta}\right) \llbracket v_{\mathcal{D}} \rrbracket_{\mathcal{D}}^{\alpha} \mathrm{d} \tau(\mathbf{x}) \mid
\end{aligned}
$$

For the following calculations, to shorten the notation, we introduce the continuous and coercive bilinear form

$$
\begin{aligned}
a_{\mathcal{D}, \xi}\left(v_{\mathcal{D}}, w_{\mathcal{D}}\right) & \left.=\int_{\Omega} \Lambda_{m} \nabla_{\mathcal{D}_{m}} v_{\mathcal{D}_{m}} \cdot \nabla_{\mathcal{D}_{m}} w_{\mathcal{D}_{m}} \mathrm{~d} \mathbf{x}+\int_{\Gamma} \Lambda_{f} \nabla_{\mathcal{D}_{f}} v_{\mathcal{D}_{f}} \cdot \nabla_{\mathcal{D}_{m}} w_{\mathcal{D}_{f}}\right) \mathrm{d} \tau_{f}(\mathbf{x}) \\
& +\sum_{i \in I} \int_{\Gamma_{i}} \frac{T_{f}}{2 \xi-1} \sum_{(\alpha, \beta) \in\left\{\left(\alpha^{ \pm}(i), \alpha^{\mp}(i)\right)\right\}}\left(\xi \llbracket w_{\mathcal{D}} \rrbracket_{\mathcal{D}}^{\alpha}+(1-\xi) \llbracket w_{\mathcal{D}} \rrbracket_{\mathcal{D}}^{\beta}\right) \llbracket v_{\mathcal{D}} \rrbracket_{\mathcal{D}}^{\alpha} \mathrm{d} \tau(\mathbf{x})
\end{aligned}
$$


for all $v_{\mathcal{D}}, w_{\mathcal{D}} \in X_{\mathcal{D}}^{0}$. Let us choose $w_{\mathcal{D}}=\left(w_{\mathcal{D}_{m}}, w_{\mathcal{D}_{f}}\right) \in X_{\mathcal{D}}^{0}$, s.t. $s\left(w_{\mathcal{D}}, u\right)=\mathcal{S}_{\mathcal{D}}(u)$. Using inequality (3.2), we derive

$$
\left|a_{\mathcal{D}, \xi}\left(v_{\mathcal{D}}, u_{\mathcal{D}}-w_{\mathcal{D}}\right)\right| \leq\left\|\left(v_{\mathcal{D}_{m}}, v_{\mathcal{D}_{f}}\right)\right\|_{\mathcal{D}} \cdot\left(\mathcal{W}_{\mathcal{D}}\left(\mathbf{q}_{m}, \mathbf{q}_{f}\right)+c s t \cdot \mathcal{S}_{\mathcal{D}}\left(u_{m}, u_{f}\right)\right) .
$$

Let us choose now $\left(v_{\mathcal{D}_{m}}, v_{\mathcal{D}_{f}}\right)=u_{\mathcal{D}}-w_{\mathcal{D}}$. Then follows, from the coercivity of $a_{\mathcal{D}, \xi}$ and by applying the definition of $\mathcal{S}_{\mathcal{D}}\left(u_{m}, u_{f}\right)$ on the left hand side of the inequality, that holds

$$
\begin{aligned}
& \left\|\nabla u_{m}-\nabla_{\mathcal{D}_{m}} u_{\mathcal{D}_{m}}\right\|_{L^{2}(\Omega)^{d}}+\left\|\nabla_{\tau} u_{f}-\nabla_{\mathcal{D}_{f}} u_{\mathcal{D}_{f}}\right\|_{L^{2}(\Gamma)^{d-1}}+\sum_{\alpha \in \chi}\left\|\llbracket u_{\mathcal{D}} \rrbracket_{\mathcal{D}}^{\alpha}-\llbracket u \rrbracket^{\alpha}\right\|_{L^{2}\left(\Gamma_{\alpha}\right)} \\
& \leq C \cdot\left(\mathcal{S}_{\mathcal{D}}\left(u_{m}, u_{f}\right)+\mathcal{W}_{\mathcal{D}}\left(\mathbf{q}_{m}, \mathbf{q}_{f}\right)\right)
\end{aligned}
$$

with a constant $C>0$ depending only on $\xi, \underline{\lambda}_{m}, \underline{\lambda}_{f}, \bar{\lambda}_{m}, \bar{\lambda}_{f}, \underline{d}_{f}, \bar{d}_{f}, \underline{\lambda}_{f, n}$, and $\bar{\lambda}_{f, n}$. Taking into account the definition of the coercivity constant $\mathcal{C}_{\mathcal{D}}$ leads to the statement of the proposition.

\section{Two Examples of Gradient Schemes}

Following [14], we consider generalised polyhedral meshes of $\Omega$. Let $\mathcal{M}$ be the set of cells that are disjoint open subsets of $\Omega$ such that $\bigcup_{K \in \mathcal{M}} \bar{K}=\bar{\Omega}$. For all $K \in \mathcal{M}, \mathbf{x}_{K}$ denotes the so-called "center" of the cell $K$ under the assumption that $K$ is star-shaped with respect to $\mathbf{x}_{K}$. Let $\mathcal{F}$ denote the set of faces of the mesh. The faces are not assumed to be planar for the VAG discretization, hence the term "generalised polyhedral cells", but they need to be planar for the HFV discretization. We denote by $\mathcal{V}$ the set of vertices of the mesh. Let $\mathcal{V}_{K}, \mathcal{F}_{K}, \mathcal{V}_{\sigma}$ respectively denote the set of the vertices of $K \in \mathcal{M}$, faces of $K$, and vertices of $\sigma \in \mathcal{F}$. For any face $\sigma \in \mathcal{F}_{K}$, we have $\mathcal{V}_{\sigma} \subset \mathcal{V}_{K}$. Let $\mathcal{M}_{s}$ (resp. $\mathcal{F}_{s}$ ) denote the set of the cells (resp. faces) sharing the vertex $s \in \mathcal{V}$. The set of edges of the mesh is denoted by $\mathcal{E}$ and $\mathcal{E}_{\sigma}$ denotes the set of edges of the face $\sigma \in \mathcal{F}$. Let $\mathcal{F}_{e}$ denote the set of faces sharing the edge $e \in \mathcal{E}$, and $\mathcal{M}_{\sigma}$ denote the set of cells sharing the face $\sigma \in \mathcal{F}$. We denote by $\mathcal{F}_{\text {ext }}$ the subset of faces $\sigma \in \mathcal{F}$ such that $\mathcal{M}_{\sigma}$ has only one element, and we set $\mathcal{E}_{\text {ext }}=\bigcup_{\sigma \in \mathcal{F}_{\text {ext }}} \mathcal{E}_{\sigma}$, and $\mathcal{V}_{\text {ext }}=\bigcup_{\sigma \in \mathcal{F}_{\text {ext }}} \mathcal{V}_{\sigma}$. The mesh is assumed to be conforming in the sense that for all $\sigma \in \mathcal{F} \backslash \mathcal{F}_{\text {ext }}$, the set $\mathcal{M}_{\sigma}$ contains exactly two cells. It is assumed that for each face $\sigma \in \mathcal{F}$, there exists a so-called "center" of the face $\mathbf{x}_{\sigma}$ such that

$$
\mathbf{x}_{\sigma}=\sum_{s \in \mathcal{V}_{\sigma}} \beta_{\sigma, s} \mathbf{x}_{s}, \text { with } \sum_{s \in \mathcal{V}_{\sigma}} \beta_{\sigma, s}=1,
$$

where $\beta_{\sigma, s} \geq 0$ for all $s \in \mathcal{V}_{\sigma}$. The face $\sigma$ is assumed to match with the union of the triangles $T_{\sigma, e}$ defined by the face center $\mathbf{x}_{\sigma}$ and each of its edge $e \in \mathcal{E}_{\sigma}$.

The mesh is assumed to be conforming w.r.t. the fracture network $\Gamma$ in the sense that there exist subsets $\mathcal{F}_{\Gamma_{i}}, i \in I$ of $\mathcal{F}$ such that

$$
\bar{\Gamma}_{i}=\bigcup_{\sigma \in \mathcal{F}_{\Gamma_{i}}} \bar{\sigma} .
$$

We will denote by $\mathcal{F}_{\Gamma}$ the set of fracture faces $\bigcup_{i \in I} \mathcal{F}_{\Gamma_{i}}$. Similarly, we will denote by $\mathcal{E}_{\Gamma}$ the set of fracture edges $\bigcup_{\sigma \in \mathcal{F}_{\Gamma}} \mathcal{E}_{\sigma}$ and by $\mathcal{V}_{\Gamma}$ the set of fracture vertices $\bigcup_{\sigma \in \mathcal{F}_{\Gamma}} \mathcal{V}_{\sigma}$.

We also define a submesh $\mathcal{T}$ of tetrahedra, where each tetrahedron $D_{K, \sigma, e}$ is the convex hull of the cell center $\mathbf{x}_{K}$ of $K$, the face center $\mathbf{x}_{\sigma}$ of $\sigma \in \mathcal{F}_{K}$ and the edge $e \in \mathcal{E}_{\sigma}$. Similarly we define a triangulation $\Delta$ of $\Gamma$, such that we have:

$$
\mathcal{T}=\bigcup_{K \in \mathcal{F}, \sigma \in \mathcal{F}_{K}, e \in \mathcal{E}_{\sigma}}\left\{D_{K, \sigma, e}\right\} \quad \text { and } \quad \Delta=\bigcup_{\sigma \in \mathcal{F}_{\Gamma}, e \in \mathcal{E}_{\sigma}}\left\{T_{\sigma, e}\right\}
$$


We introduce for $D \in \mathcal{T}$ the diameter $h_{D}$ of $D$ and set $h_{\mathcal{T}}=\max _{D \in \mathcal{T}} h_{D}$. The regularity of our polyhedral mesh will be measured by the shape regularity of the tetrahedral submesh defined by $\theta_{\mathcal{T}}=\max _{D \in \mathcal{T}} \frac{h_{D}}{\rho_{D}}$ where $\rho_{D}$ is the insphere diameter of $D \in \mathcal{T}$.

The set of matrix $\times$ fracture degrees of freedom is denoted by $d o f_{\mathcal{D}_{m}} \times d o f_{\mathcal{D}_{f}}$. The real vector spaces $X_{\mathcal{D}_{m}}$ and $X_{\mathcal{D}_{f}}$ of discrete unknowns in the matrix and in the fracture network respectively are then defined by

$$
\begin{aligned}
X_{\mathcal{D}_{m}} & =\operatorname{span}\left\{\mathfrak{e}_{\nu} \mid \nu \in \operatorname{dof}_{\mathcal{D}_{m}}\right\} \\
X_{\mathcal{D}_{f}} & =\operatorname{span}\left\{\mathfrak{e}_{\nu} \mid \nu \in \operatorname{dof}_{\mathcal{D}_{f}}\right\},
\end{aligned}
$$

where

$$
\mathfrak{e}_{\nu}= \begin{cases}\left(\delta_{\nu \mu}\right)_{\mu \in \operatorname{dof}_{\mathcal{D}_{m}}} & \text { for } \nu \in \operatorname{dof}_{\mathcal{D}_{m}} \\ \left(\delta_{\nu \mu}\right)_{\mu \in \text { dof }_{\mathcal{D}_{f}}} & \text { for } \nu \in \operatorname{dof}_{\mathcal{D}_{f}}\end{cases}
$$

For $u_{\mathcal{D}_{m}} \in X_{\mathcal{D}_{m}}$ and $\nu \in \operatorname{dof}_{\mathcal{D}_{m}}$ we denote by $u_{\nu}$ the $\nu$ th component of $u_{\mathcal{D}_{m}}$ and likewise for $u_{\mathcal{D}_{f}} \in X_{\mathcal{D}_{f}}$ and $\nu \in d o f_{\mathcal{D}_{f}}$. We also introduce the product of these vector spaces

$$
X_{\mathcal{D}}=X_{\mathcal{D}_{m}} \times X_{\mathcal{D}_{f}}
$$

for which we have $\operatorname{dim} X_{\mathcal{D}}=\# \operatorname{dof} f_{\mathcal{D}_{m}}+\# d o f_{\mathcal{D}_{f}}$. To account for zero boundary conditions on $\partial \Omega$ and $\Sigma_{0}$ we introduce the subsets $\operatorname{dof}_{\operatorname{Dir}_{m}} \subset d o f_{\mathcal{D}_{m}}$, and $d o f_{D_{i r_{f}}} \subset \operatorname{dof}_{\mathcal{D}_{f}}$, and we set $d o f_{\text {Dir }}=d o f_{\text {Dirm }_{m}} \times d o f_{D_{i r_{f}}}$, and

$$
X_{\mathcal{D}}^{0}=\left\{u \in X_{\mathcal{D}} \mid u_{\nu}=0 \text { for all } \nu \in \operatorname{dof}_{\text {Dir }}\right\} .
$$

\subsection{Vertex Approximate Gradient Discretization}

In this subsection, the VAG discretization introduced in [14] for diffusive problems on heterogeneous anisotropic media is extended to the hybrid dimensional model. We consider the $\mathbb{P}_{1}$ finite element construction as well as a finite volume version using lumping both for the source terms and the matrix fracture fluxes.

We first establish an equivalence relation on each $\mathcal{M}_{s}, s \in \mathcal{V}$, by

$$
\begin{aligned}
K \equiv_{\mathcal{M}_{s}} L \Longleftrightarrow \quad & \text { there exists } n \in \mathbb{N} \text { and a sequence }\left(\sigma_{i}\right)_{i=1, \ldots, n} \text { in } \mathcal{F}_{s} \backslash \mathcal{F}_{\Gamma} \\
& \text { such that } K \in \mathcal{M}_{\sigma_{1}}, L \in \mathcal{M}_{\sigma_{n}} \text { and } \mathcal{M}_{\sigma_{i+1}} \cap \mathcal{M}_{\sigma_{i}} \neq \emptyset \\
& \text { for } i=1, \ldots, n-1 .
\end{aligned}
$$

Let us then denote by $\overline{\mathcal{M}}_{s}$ the set of all classes of equivalence of $\mathcal{M}_{s}$ and by $\bar{K}_{s}$ the element of $\overline{\mathcal{M}}_{s}$ containing $K \in \mathcal{M}$. Obviously $\overline{\mathcal{M}}_{s}$ might have more than one element only if $s \in \mathcal{V}_{\Gamma}$. Then we define (cf. Figure 2)

$$
\begin{aligned}
\operatorname{dof}_{\mathcal{D}_{m}} & =\mathcal{M} \cup\left\{K_{\sigma} \mid \sigma \in \mathcal{F}_{\Gamma}, K \in \mathcal{M}_{\sigma}\right\} \cup\left\{\bar{K}_{s} \mid s \in \mathcal{V}, \bar{K}_{s} \in \overline{\mathcal{M}}_{s}\right\}, \\
\operatorname{dof}_{\mathcal{D}_{f}} & =\mathcal{F}_{\Gamma} \cup \mathcal{V}_{\Gamma}, \\
\operatorname{dof}_{\text {Dirm }_{m}} & :=\left\{\bar{K}_{s} \mid s \in \mathcal{V}_{\text {ext }}, \bar{K}_{s} \in \overline{\mathcal{M}}_{s}\right\}, \\
\operatorname{dof}_{\text {Dirf }} & =\mathcal{V}_{\Gamma} \cap \mathcal{V}_{\text {ext }} .
\end{aligned}
$$


We thus have

$$
\begin{aligned}
X_{\mathcal{D}_{m}} & =\left\{u_{K} \mid K \in \mathcal{M}\right\} \cup\left\{u_{K_{\sigma}} \mid \sigma \in \mathcal{F}_{\Gamma}, K \in \mathcal{M}_{\sigma}\right\} \\
& \cup\left\{u_{\bar{K}_{s}} \mid s \in \mathcal{V}, \bar{K}_{s} \in \overline{\mathcal{M}}_{s}\right\}, \\
X_{\mathcal{D}_{f}} & =\left\{u_{\sigma} \mid \sigma \in \mathcal{F}_{\Gamma}\right\} \cup\left\{u_{s} \mid s \in \mathcal{V}_{\Gamma}\right\} .
\end{aligned}
$$

Now we can introduce the piecewise affin reconstruction operators

$$
\Pi_{\mathcal{T}}: X_{\mathcal{D}_{m}} \longrightarrow H^{1}(\Omega \backslash \bar{\Gamma}) \quad \text { and } \quad \Pi_{\Delta}: X_{\mathcal{D}_{f}} \longrightarrow H^{1}(\Gamma),
$$

which act linearly on $X_{\mathcal{D}_{m}}$ and $X_{\mathcal{D}_{f}}$, and are such that $\Pi_{\mathcal{T}} u_{\mathcal{D}_{m}}$ is affine on each $D_{K, \sigma, e} \in \mathcal{T}$ and satisfies on each cell $K \in \mathcal{M}$

$$
\begin{array}{lr}
\Pi_{\mathcal{T} u_{\mathcal{D}_{m}}\left(\mathbf{x}_{K}\right)=u_{K},}, & \forall s \in \mathcal{V}_{K}, \\
\Pi_{\mathcal{T}} u_{\mathcal{D}_{m}}\left(\mathbf{x}_{s}\right)=u_{\bar{K}_{s}} & \forall \sigma \in \mathcal{F}_{K} \cap \mathcal{F}_{\Gamma}, \\
\prod_{\mathcal{T}} u_{\mathcal{D}_{m}}\left(\mathbf{x}_{\sigma}\right)=u_{K_{\sigma}} & \forall \sigma \in \mathcal{F}_{K} \backslash \mathcal{F}_{\Gamma}, \\
\prod_{\mathcal{T}} u_{\mathcal{D}_{m}}\left(\mathbf{x}_{\sigma}\right)=\sum_{s \in \mathcal{V}_{\sigma}} \beta_{\sigma, s} u_{\bar{K}_{s}} & \forall \sigma
\end{array}
$$

while $\Pi_{\Delta} u_{\mathcal{D}_{f}}$ is affine on each $T_{\sigma, e} \in \Delta$ and satisfies for all $\nu \in d o f_{\mathcal{D}_{f}}$

$$
\Pi_{\Delta} u_{\mathcal{D}_{f}}\left(\mathbf{x}_{\nu}\right)=u_{\nu}
$$

where $\mathbf{x}_{\nu} \in \bar{\Omega}$ is the grid point associated with the degree of freedom $\nu \in \operatorname{dof}_{\mathcal{D}_{m}} \cup \operatorname{dof} f_{\mathcal{D}_{f}}$.

The discrete gradients on $X_{\mathcal{D}_{m}}$ and $X_{\mathcal{D}_{f}}$ are subsequently defined by

$$
\nabla_{\mathcal{D}_{m}}=\nabla \Pi_{\mathcal{T}} \quad \text { and } \quad \nabla_{\mathcal{D}_{f}}=\nabla_{\tau} \Pi_{\Delta}
$$

The so defined reconstruction operators and discrete gradients correspond to the $V^{0}$ conforming $\mathbb{P}_{1}$ Finite Element reconstructions on a tetrahedral submesh using barycentric interpolation to eliminate the d.o.f. at faces $\sigma \in \mathcal{F}_{K} \backslash \mathcal{F}_{\Gamma}$.
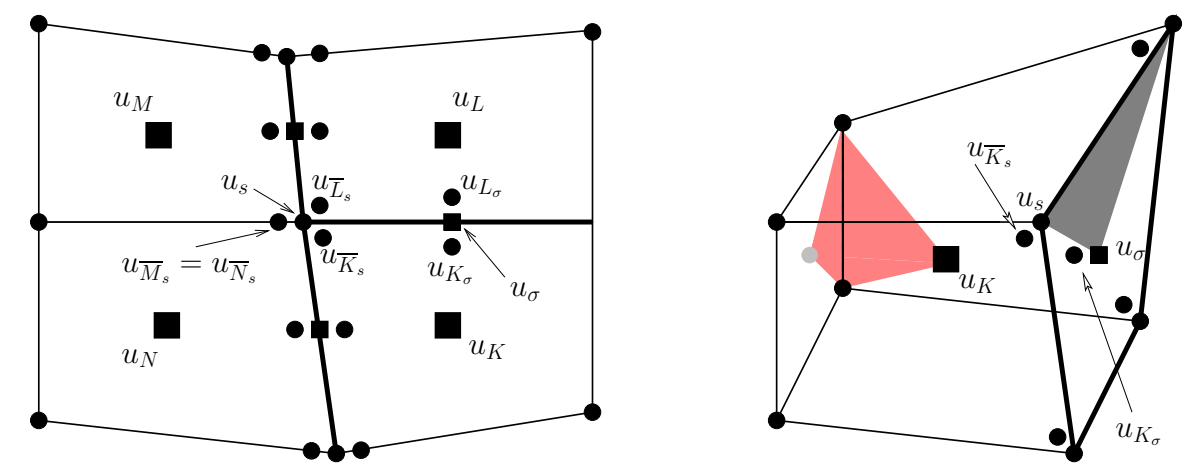

Figure 2: Left: Illustration of d.o.f. in 2D for four cells intersected by three fractures (thick lines).

Right: $3 \mathrm{D}$ cell $K$ touching a fracture face $\sigma$. Illustration of the simplices on which $\nabla_{\mathcal{D}_{m}}$ is constant (red) and $\nabla_{\mathcal{D}_{f}}$ is constant (grey).

We define VAG-FE scheme's reconstruction operators by

- $\Pi_{\mathcal{D}_{m}}=\Pi_{\mathcal{T}}$,

- $\Pi_{\mathcal{D}_{f}}=\Pi_{\Delta}$,

- $\llbracket \cdot \rrbracket_{\mathcal{D}}^{\alpha}=\gamma_{\alpha} \Pi_{\mathcal{T}}-\Pi_{\Delta} \quad$ for all $\alpha \in \chi$. 
For the family of VAG-CV schemes, reconstruction operators are piecewise constant. For $K \in \mathcal{M}$ let $\operatorname{dof}_{K}=\left\{\bar{K}_{s}, s \in \mathcal{V}_{K}\right\} \cup\left\{K_{\sigma}, \sigma \in \mathcal{F}_{K} \cap \mathcal{F}_{\Gamma}\right\}$. Analogously, in the fracture domain, for $\sigma \in \mathcal{F}_{\Gamma}$ let $d o f_{\sigma}=\mathcal{V}_{\sigma}$. We introduce, for any given $K \in \mathcal{M}$, a partition $\left\{\omega_{K}^{\nu}\right\}_{\nu \in\{K\} \cup d o f_{K} \backslash \text { dof }}$ Dir $_{\text {D }}$ of $K$. Similarly, we define for any given $\sigma \in \mathcal{F}_{\Gamma}$ a partition $\left\{\omega_{\sigma}^{\nu}\right\}_{\nu \in\{\sigma\} \cup d o f_{\sigma} \backslash d o f_{D i r}}$ of $\sigma$. For each $\nu \in d o f_{\mathcal{D}_{m}}$, we define the open set $\omega_{\nu}=\operatorname{int}\left(\bigcup_{K \in \mathcal{M}} \bar{\omega}_{K}^{\nu}\right)$, with the convention $\omega_{K}^{\nu}=\emptyset$, if $\nu \notin d o f_{K}$. For each $\nu \in d o f_{\mathcal{D}_{f}}$, we define the open set $\omega_{\nu}=\operatorname{int}\left(\bigcup_{\sigma \in \mathcal{F}} \bar{\omega}_{\sigma}^{\nu}\right)$, where $\omega_{\sigma}^{\nu}=\emptyset$,

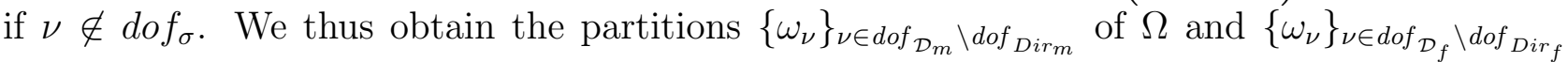
of $\Gamma$. We also introduce for each $T=T_{\sigma, s, s^{\prime}} \in \Delta$ a partition $\left\{T_{i}\right\}_{i=1, \ldots, 3}$ of $T$, which we need for the definition of the VAG-CV matrix-fracture interaction operators. We assume that holds $\left|T_{1}\right|=\left|T_{2}\right|=\left|T_{3}\right|=\frac{1}{3}|T|$ in order to preserve the first order accuracy of the scheme. The VAG-CV discretization is particularly adapted for the treatment of multiphase flow processes through heterogeneous media (cf. [16]). Provided the mesh is comforming w.r.t. the heterogeneous layers, we can assume that key geological quantities are constant per cell and per fracture face. Therefore, in the numerical scheme, we do not need to reconstruct the just introduced partitions explicitly, but only have to define their corresponding volumes. Finally, we need a mapping between the degrees of freedom of the matrix domain, which are situated on one side of the fracture network, and the set of indices $\chi$. For $K_{\sigma} \in d o f_{\mathcal{D}_{m}}$ we have the one-element set $\chi\left(K_{\sigma}\right)=\left\{\alpha \in \chi \mid\left(\mathbf{x}_{K}-\mathbf{x}_{\sigma}\right) \cdot \mathbf{n}_{\alpha}<0\right\}$ and therefore the notation $\alpha\left(K_{\sigma}\right)=\alpha \in \chi\left(K_{\sigma}\right)$.

The VAG-CV scheme's reconstruction operators are

$$
\begin{aligned}
& \text { - } \Pi_{\mathcal{D}_{m}} u_{\mathcal{D}_{m}}=\sum_{\nu \in \text { dof }_{\mathcal{D}_{m} \backslash \text { dof }_{\text {Dirm }}}} u_{\nu} \mathbb{1}_{\omega_{\nu}}, \\
& \text { - } \Pi_{\mathcal{D}_{f}} u_{\mathcal{D}_{f}}=\sum_{\nu \in \operatorname{dof}_{\mathcal{D}_{f}} \backslash \operatorname{dof}_{\text {Dir }_{f}}} u_{\nu} \mathbb{1}_{\omega_{\nu}}, \\
& \text { - } \llbracket u_{\mathcal{D}} \rrbracket_{\mathcal{D}}^{\alpha}=\sum_{T_{\sigma, s, s^{\prime}} \in \Delta} \sum_{K \in \mathcal{M}_{\sigma}}\left(\left(u_{K_{\sigma}}-u_{\sigma}\right) \mathbb{1}_{T_{1}}+\left(u_{\bar{K}_{s}}-u_{s}\right) \mathbb{1}_{T_{2}}+\left(u_{\bar{K}_{s^{\prime}}}-u_{s^{\prime}}\right) \mathbb{1}_{T_{3}}\right) \delta_{\alpha\left(K_{\sigma}\right) \alpha} \mathbb{1}_{\Gamma_{\alpha}} .
\end{aligned}
$$

Remark 4.1 The VAG-CV scheme leads us to recover fluxes for the matrix-fracture interactions involving degrees of freedom located at the same physical point (see appendix section $B)$.

Proposition 4.1 Let us consider a sequence of meshes $\left(\mathcal{M}^{l}\right)_{l \in \mathbb{N}}$ and let us assume that the sequence $\left(\mathcal{T}^{l}\right)_{l \in \mathbb{N}}$ of tetrahedral submeshes is shape regular, i.e. $\theta_{\mathcal{T}^{l}}$ is uniformly bounded. We also assume that $\lim _{l \rightarrow \infty} h_{\mathcal{T}^{l}}=0$. Then, the corresponding sequence of gradient discretizations $\left(\mathcal{D}^{l}\right)_{l \in \mathbb{N}}$, defined by (4.1), (4.2), (4.3), is coercive, consistent and limit conforming.

Proof The $V A G-F E$ scheme's reconstruction operators are conforming in the sense that $\nabla_{\mathcal{D}_{m}}=$ $\nabla \Pi_{\mathcal{D}_{m}}, \nabla_{\mathcal{D}_{f}}=\nabla_{\tau} \Pi_{\mathcal{D}_{f}}, \llbracket \cdot \rrbracket_{\mathcal{D}}^{\alpha} \neq \llbracket \Pi_{\mathcal{D}_{m}}, \Pi_{\mathcal{D}_{f}} \rrbracket^{\alpha}$. Therefore we deduce coercivity from Proposition 2.1. Furthermore, we have by partial integration $\mathcal{W}_{\mathcal{D}}\left(\mathbf{q}_{m}, \mathbf{q}_{f}\right)=0$ for all $\left(\mathbf{q}_{m}, \mathbf{q}_{f}\right) \in W$. Hence $\left(\mathcal{D}^{l}\right)_{l \in \mathbb{N}}$ is limit conforming.

To prove consistency, we need the following prerequisites. We define the linear mapping $P_{\mathcal{D}_{m}}: C_{\Omega}^{\infty} \rightarrow X_{\mathcal{D}_{m}}^{0}$ such that for all $\psi_{m} \in C_{\Omega}^{\infty}$ and any $\nu \in d o f_{\mathcal{D}_{m}}$ holds $\left(P_{\mathcal{D}_{m}} \psi_{m}\right)_{\nu}=\psi_{m}\left(\mathbf{x}_{\nu}\right)$. Likewise, we define the linear mapping $P_{\mathcal{D}_{f}}: C_{\Gamma}^{\infty} \rightarrow X_{\mathcal{D}_{f}}^{0}$ such that for all $\psi_{f} \in C_{\Gamma}^{\infty}$ holds $\left(P_{\mathcal{D}_{f}} \psi_{f}\right)_{\nu}=\psi_{f}\left(\mathbf{x}_{\nu}\right)$ for all $\nu \in d o f_{\mathcal{D}_{f}}$. It follows from the classical Finite Element approximation theory and from the fact that the interpolation $\sum_{s \in \mathcal{V}_{\sigma}} \beta_{\sigma, s}\left(P_{\mathcal{D}_{m}} \psi_{m}\right)_{\bar{K}_{s}}$ at the point $\mathbf{x}_{\sigma}, \sigma \in \mathcal{F}_{K} \backslash \mathcal{F}_{\Gamma}$ is exact on cellwise affine functions, that for all $\left(\psi_{m}, \psi_{f}\right) \in C_{\Omega}^{\infty} \times C_{\Gamma}^{\infty}$ holds

$$
\left\|\Pi_{\mathcal{T}} P_{\mathcal{D}_{m}} \psi_{m}-\psi_{m}\right\|_{H^{1}(\Omega \backslash \bar{\Gamma})}+\left\|\Pi_{\Delta} P_{\mathcal{D}_{f}} \psi_{f}-\psi_{f}\right\|_{H^{1}(\Gamma)} \leq C\left(\psi_{m}, \psi_{f}, \theta_{\mathcal{T}}\right) h_{\mathcal{T}} .
$$


The trace inequality implies that for all $v \in H_{\partial \Omega}^{1}(\Omega \backslash \bar{\Gamma})$ holds

$$
\left\|\gamma_{\alpha} v\right\|_{L^{2}\left(\Gamma_{\alpha}\right)} \leq C(\Omega \backslash \bar{\Gamma})\|v\|_{H^{1}(\Omega \backslash \bar{\Gamma})} \quad \text { for } \alpha \in \chi
$$

We can then calculate for $\left(u_{m}, u_{f}\right) \in C_{\Omega}^{\infty} \times C_{\Gamma}^{\infty}$ :

$$
\begin{aligned}
\mathcal{S}_{\mathcal{D}}\left(u_{m}, u_{f}\right) & \leq \sqrt{2}\left\|\Pi_{\mathcal{T}} P_{\mathcal{D}_{m}} u_{m}-u_{m}\right\|_{H^{1}(\Omega \backslash \bar{\Gamma})}+\sum_{\alpha \in \chi}\left\|\gamma_{\alpha}\left(\Pi_{\mathcal{T}} P_{\mathcal{D}_{m}} u_{m}-u_{m}\right)\right\|_{L^{2}\left(\Gamma_{\alpha}\right)} \\
& +\sum_{i \in I} \sqrt{8}\left\|\Pi_{\Delta} P_{\mathcal{D}_{f}} u_{f}-u_{f}\right\|_{H^{1}\left(\Gamma_{i}\right)} \leq C\left(\Omega \backslash \bar{\Gamma},\left(u_{m}, u_{f}\right), \theta_{\mathcal{T}}\right) h_{\mathcal{T}} .
\end{aligned}
$$

Since $C_{\Omega}^{\infty} \times C_{\Gamma}^{\infty}$ is dense in $V^{0}$, the sequence of $V A G-F E$ discretisations $\left(\mathcal{D}_{m}^{l}\right)_{l \in \mathbb{N}}$ is consistent if $h_{\mathcal{T}^{l}} \rightarrow 0$ and $\theta_{\mathcal{T}^{l}}$ is bounded for $l \rightarrow \infty$.

Proposition 4.2 Let us consider a sequence of meshes $\left(\mathcal{M}^{l}\right)_{l \in \mathbb{N}}$ and let us assume that the sequence $\left(\mathcal{T}^{l}\right)_{l \in \mathbb{N}}$ of tetrahedral submeshes is shape regular, i.e. $\theta_{\mathcal{T}^{l}}$ is uniformly bounded. We also assume that $\lim _{l \rightarrow \infty} h_{\mathcal{T}^{l}}=0$. Then, any corresponding sequence of gradient discretizations $\left(\mathcal{D}^{l}\right)_{l \in \mathbb{N}}$, defined by (4.1), (4.2), (4.4), is coercive, consistent and limit conforming.

Proof For this proof, let us denote by $\left(\mathcal{D}^{l}\right)_{l \in \mathbb{N}}$ the sequence of $V A G-C V$ gradient discretisations and by $\left(\overline{\mathcal{D}}^{l}\right)_{l \in \mathbb{N}}=\left(X_{\mathcal{D}^{l}}, \Pi_{\overline{\mathcal{D}}_{m}^{l}}, \Pi_{\overline{\mathcal{D}}_{f}^{l}}, \llbracket \cdot \rrbracket_{\overline{\mathcal{D}}^{l}}^{\alpha}, \nabla_{\mathcal{D}_{m}^{l}}, \nabla_{\mathcal{D}_{f}^{l}}\right)_{l \in \mathbb{N}}$ the corresponding sequence of $V A G-F E$ gradient discretisations. It can be verified that [8], Lemma 3.4 applies to our case, both, in the matrix domain, where face unknowns might occur, as well as in the fracture network, a domain of codimension 1. This means that we can state that there exist constants $C_{m}\left(\theta_{\mathcal{T}}\right), C_{f}\left(\theta_{\mathcal{T}}\right)>0$, such that

$$
\begin{aligned}
\left\|\Pi_{\overline{\mathcal{D}}_{m}} u_{\mathcal{D}_{m}}-\Pi_{\mathcal{D}_{m}} u_{\mathcal{D}_{m}}\right\|_{L^{2}(\Omega)} & \leq C_{m} h_{\mathcal{T}}\left\|\nabla_{\mathcal{D}_{m}} u_{\mathcal{D}_{m}}\right\|_{L^{2}(\Omega)^{d}} \quad \text { and } \\
\left\|\Pi_{\overline{\mathcal{D}}_{f}} u_{\mathcal{D}_{f}}-\Pi_{\mathcal{D}_{f}} u_{\mathcal{D}_{f}}\right\|_{L^{2}(\Gamma)} & \leq C_{f} h_{\Delta}\left\|\nabla_{\mathcal{D}_{f}} u_{\mathcal{D}_{f}}\right\|_{L^{2}(\Gamma)^{d-1}}
\end{aligned}
$$

Coercivity: It is easy to check that

$$
\left\|\llbracket u_{\mathcal{D}} \rrbracket \frac{\alpha}{\mathcal{D}}\right\|_{L^{2}\left(\Gamma_{\alpha}\right)} \leq \sqrt{3}\left\|\llbracket u_{\mathcal{D}} \rrbracket_{\mathcal{D}}^{\alpha}\right\|_{L^{2}\left(\Gamma_{\alpha}\right)}
$$

and therefore $\left\|u_{\mathcal{D}}\right\|_{\overline{\mathcal{D}}} \leq C\left\|u_{\mathcal{D}}\right\|_{\mathcal{D}}$, for a $C>0$. Coercivity for $\left(\overline{\mathcal{D}}^{l}\right)_{l \in \mathbb{N}}$ can then be deduced from coercivity for $\left(\mathcal{D}^{l}\right)_{l \in \mathbb{N}}$ via the inequality

$$
\begin{aligned}
\left\|\Pi_{\mathcal{D}_{m}} u_{\mathcal{D}_{m}}\right\|_{L^{2}(\Omega)}+\left\|\Pi_{\mathcal{D}_{f}} u_{\mathcal{D}_{f}}\right\|_{L^{2}(\Gamma)} & \leq\left\|\Pi_{\overline{\mathcal{D}}_{m}} u_{\mathcal{D}_{m}}\right\|_{L^{2}(\Omega)}+\left\|\Pi_{\overline{\mathcal{D}}_{f}} u_{\mathcal{D}_{f}}\right\|_{L^{2}(\Gamma)} \\
& +\left(\left\|\nabla_{\mathcal{D}_{m}} u_{\mathcal{D}_{m}}\right\|_{L^{2}(\Omega)^{d}}+\left\|\nabla_{\mathcal{D}_{f}} u_{\mathcal{D}_{f}}\right\|_{L^{2}(\Gamma)^{d-1}}\right) \cdot \mathcal{O}\left(h_{\mathcal{T}}\right),
\end{aligned}
$$

where we have used (4.6) and (4.7).

Consistency: Classically, for all $\left(\varphi_{m}, \varphi_{f}\right) \in C_{\Omega}^{\infty} \times C_{\Gamma}^{\infty}$, we have the estimate

$$
\begin{aligned}
& \left\|\Pi_{\mathcal{D}_{m}} P_{\mathcal{D}_{m}} \varphi_{m}-\varphi_{m}\right\|_{L^{2}(\Omega)}+\left\|\Pi_{\mathcal{D}_{f}} P_{\mathcal{D}_{f}} \varphi_{f}-\varphi_{f}\right\|_{L^{2}(\Gamma)} \\
& +\sum_{\alpha \in \chi}\left\|\llbracket\left(P_{\mathcal{D}_{m}} \varphi_{m}, P_{\mathcal{D}_{f}} \varphi_{f}\right) \rrbracket_{\mathcal{D}}^{\alpha}-\llbracket\left(\varphi_{m}, \varphi_{f}\right) \rrbracket^{\alpha}\right\|_{L^{2}\left(\Gamma_{\alpha}\right)} \leq \operatorname{cst}\left(\varphi_{m}, \varphi_{f}\right) \cdot h_{\mathcal{T}},
\end{aligned}
$$

while (4.5) grants that holds

$$
\left\|\nabla_{\mathcal{D}_{m}} P_{\mathcal{D}_{m}} \varphi_{m}-\nabla \varphi\right\|_{L^{2}(\Omega)}+\left\|\nabla_{\mathcal{D}_{f}} P_{\mathcal{D}_{f}} \varphi_{f}-\nabla \varphi\right\|_{L^{2}(\Gamma)} \leq \operatorname{cst}\left(\varphi_{m}, \varphi_{f}, \theta_{\mathcal{T}}\right) h_{\mathcal{T}} .
$$


Taking into account that $C_{\Omega}^{\infty} \times C_{\Gamma}^{\infty}$ is dense in $V$, we see that the treated discretisation is consistent with $\mathcal{S}_{\mathcal{D}}\left(\varphi_{m}, \varphi_{f}\right)=\mathcal{O}\left(h_{\mathcal{T}}\right)$ for $\left(\varphi_{m}, \varphi_{f}\right) \in C_{\Omega}^{\infty} \times C_{\Gamma}^{\infty}$.

Limit Conformity: For all $T \in \Delta$ and for all $u_{\mathcal{D}}=\left(u_{\mathcal{D}_{m}}, u_{\mathcal{D}_{f}}\right) \in X_{\mathcal{D}_{m}}$ we have

$$
\int_{T}\left(\llbracket u_{\mathcal{D}} \rrbracket \frac{\alpha}{\mathcal{D}}-\llbracket u_{\mathcal{D}} \rrbracket_{\mathcal{D}}^{\alpha}\right) \mathrm{d} \tau(\mathbf{x})=0 .
$$

Introducing the linear operator $P: L^{2}\left(\Gamma_{\alpha}\right) \rightarrow L^{2}\left(\Gamma_{\alpha}\right)$ such that $P(\varphi)=\frac{1}{|T|} \int_{T} \varphi \mathrm{d} \tau(\mathbf{x})$ on $T$ for all $T \in \Delta$, we first calculate for any $\mathbf{q}_{m} \in C_{W_{m}}^{\infty}$

$$
\left\|\gamma_{\mathbf{n}, \alpha} \mathbf{q}_{m}-P\left(\gamma_{\mathbf{n}, \alpha} \mathbf{q}_{m}\right)\right\|_{L^{2}\left(\Gamma_{\alpha}\right)}^{2}=\sum_{\sigma \in \mathcal{F}_{\alpha}}\left(\sum_{T \in \Delta \text { s.t. } T \subset \sigma}\left\|\gamma_{\mathbf{n}, \alpha} \mathbf{q}_{m}-P\left(\gamma_{\mathbf{n}, \alpha} \mathbf{q}_{m}\right)\right\|_{L^{2}(T)}^{2}\right) \leq C\left(\mathbf{q}_{m}, \theta_{\mathcal{T}}\right) \cdot h_{\mathcal{T}}^{2} .
$$

For the discrete part, we are able to derive (taking into account [8], Lemma 3.4)

$$
\left\|\llbracket u_{\mathcal{D}} \rrbracket \frac{\alpha}{\mathcal{D}}-\llbracket u_{\mathcal{D}} \rrbracket_{\mathcal{D}}^{\alpha}\right\|_{L^{2}\left(\Gamma_{\alpha}\right)}^{2} \leq C h_{\Delta}\left(\left\|\nabla_{\mathcal{D}_{m}} u_{\mathcal{D}_{m}}\right\|_{L^{2}(\Omega)^{d}}^{2}+\left\|\nabla_{\mathcal{D}_{f}} u_{\mathcal{D}_{f}}\right\|_{L^{2}(\Gamma)^{d-1}}^{2}\right) .
$$

with a constant $C$ depending only on $\theta_{\mathcal{T}}$. We can thus proceed

$$
\begin{aligned}
\mid \int_{\Gamma_{\alpha}} \gamma_{\mathbf{n}, \alpha} \mathbf{q}_{m}\left(\llbracket u_{\mathcal{D}} \rrbracket \frac{\alpha}{\mathcal{D}}\right. & \left.-\llbracket u_{\mathcal{D}} \rrbracket_{\mathcal{D}}^{\alpha}\right) \mathrm{d} \tau(\mathbf{x})|=| \int_{\Gamma_{\alpha}}\left(\gamma_{\mathbf{n}, \alpha} \mathbf{q}_{m}-P\left(\gamma_{\mathbf{n}, \alpha} \mathbf{q}_{m}\right)\right)\left(\llbracket u_{\mathcal{D}} \rrbracket_{\mathcal{D}}^{\alpha}-\llbracket u_{\mathcal{D}} \rrbracket_{\mathcal{D}}^{\alpha}\right) \mathrm{d} \tau(\mathbf{x}) \mid \\
& \leq\left\|\gamma_{\mathbf{n}, \alpha} \mathbf{q}_{m}-P\left(\gamma_{\mathbf{n}, \alpha} \mathbf{q}_{m}\right)\right\|_{L^{2}\left(\Gamma_{\alpha}\right)}\left\|\llbracket u_{\mathcal{D}} \rrbracket_{\mathcal{\mathcal { D }}}^{\alpha}-\llbracket u_{\mathcal{D}} \rrbracket_{\mathcal{D}}^{\alpha}\right\|_{L^{2}\left(\Gamma_{\alpha}\right)} \\
& \leq C\left(\mathbf{q}_{m}, \theta_{\mathcal{T}}\right) h_{\mathcal{T}}^{\frac{3}{2}}\left(\left\|\nabla_{\mathcal{D}_{m}} u_{\mathcal{D}_{m}}\right\|_{L^{2}(\Omega)^{d}}^{2}+\left\|\nabla_{\mathcal{D}_{f}} u_{\mathcal{D}_{f}}\right\|_{L^{2}(\Gamma)^{d-1}}^{2}\right)^{\frac{1}{2}}
\end{aligned}
$$

for all $\mathbf{q}_{m} \in C_{W_{m}}^{\infty}$. Taking into account (4.5), (4.7) and the conformity of $\overline{\mathcal{D}}$, we can now conclude by calculating for all $\mathbf{q}=\left(\mathbf{q}_{m}, \mathbf{q}_{f}\right) \in C_{W_{m}}^{\infty} \times C_{W_{f}}^{\infty}$

$$
\begin{aligned}
\left|w_{\overline{\mathcal{D}}}\left(u_{\mathcal{D}}, \mathbf{q}\right)\right| & =\left|\left(w_{\overline{\mathcal{D}}}-w_{\mathcal{D}}\right)\left(u_{\mathcal{D}}, \mathbf{q}\right)\right|=\mid \int_{\Omega} \operatorname{divq}_{m}\left(\Pi_{\mathcal{D}_{m}}-\Pi_{\overline{\mathcal{D}}_{m}}\right) u_{\mathcal{D}_{m}} \mathrm{~d} \mathbf{x}+\int_{\Gamma} \operatorname{div}_{\tau} \mathbf{q}_{f}\left(\Pi_{\mathcal{D}_{f}}-\Pi_{\overline{\mathcal{D}}_{f}}\right) u_{\mathcal{D}_{f}} \mathrm{~d} \tau(\mathbf{x}) \\
& +\sum_{\alpha \in \chi} \int_{\Gamma_{\alpha}} \gamma_{\mathbf{n}, \alpha} \mathbf{q}_{m}\left(\left(\llbracket u_{\mathcal{D}} \rrbracket \frac{\alpha}{\mathcal{D}}-\llbracket u_{\mathcal{D}} \rrbracket_{\mathcal{D}}^{\alpha}\right)+\left(\Pi_{\overline{\mathcal{D}}_{f}}-\Pi_{\mathcal{D}_{f}}\right) u_{\mathcal{D}_{f}}\right) \mathrm{d} \tau(\mathbf{x}) \mid \\
& \leq\left\|\Pi_{\overline{\mathcal{D}}_{m}} u_{\mathcal{D}_{m}}-\Pi_{\mathcal{D}_{m}} u_{\mathcal{D}_{m}}\right\|_{L^{2}(\Omega)} \cdot\left\|\operatorname{divq_{m}}\right\|_{L^{2}(\Omega)}+\left\|\Pi_{\overline{\mathcal{D}}_{f}} u_{\mathcal{D}_{f}}-\Pi_{\mathcal{D}_{f}} u_{\mathcal{D}_{f}}\right\|_{L^{2}(\Gamma)} \cdot\left\|\operatorname{div}_{\tau} \mathbf{q}_{f}\right\|_{L^{2}(\Gamma)} \\
& +\sum_{\alpha \in \chi}\left(\left\|\Pi_{\overline{\mathcal{D}}_{f}} u_{\mathcal{D}_{f}}-\Pi_{\mathcal{D}_{f}} u_{\mathcal{D}_{f}}\right\|_{L^{2}\left(\Gamma_{\alpha}\right)}\left\|\gamma_{\mathbf{n}, \alpha} \mathbf{q}_{m}\right\|_{L^{2}\left(\Gamma_{\alpha}\right)}+\left|\int_{\Gamma_{\alpha}} \gamma_{\mathbf{n}, \alpha} \mathbf{q}_{m}\left(\llbracket u_{\mathcal{D}} \rrbracket \overline{\mathcal{D}}-\llbracket u_{\mathcal{D}} \rrbracket \mathcal{D}_{\mathcal{D}}\right)\right|\right) \\
& \leq C\left(\theta_{\mathcal{T}}, \mathbf{q}\right) \cdot h_{\mathcal{T}} \cdot\left\|u_{\mathcal{D}}\right\|_{\mathcal{D}} .
\end{aligned}
$$

Taking into account the density of $C_{W_{m}}^{\infty} \times C_{W_{f}}^{\infty}$ in $W$, the proof is complete.

Remark 4.2 The proofs of Propositions 4.1 and 4.2 show that for solutions $\left(u_{m}, u_{f}\right) \in V^{0}$ and $\left(\mathbf{q}_{m}, \mathbf{q}_{f}\right) \in W$ of $(2.1)$ such that $u_{m} \in C^{2}(\bar{K}), u_{f} \in C^{2}(\bar{\sigma}), \mathbf{q}_{m} \in\left(C^{1}(\bar{K})\right)^{d}, \mathbf{q}_{f} \in\left(C^{1}(\bar{\sigma})\right)^{d-1}$ for all $K \in \mathcal{M}$ and all $\sigma \in \Gamma_{f}$, the $V A G$ schemes are consistent and limit conforming of order 1 , and therefore convergent of order 1.

\subsection{Hybrid Finite Volume Discretization}

In this subsection, the HFV scheme introduced in [15] is extended to the hybrid dimensional

Darcy flow model. We assume here that the faces are planar and that $\mathbf{x}_{\sigma}$ is the barycenter of 
$\sigma$ for all $\sigma \in \mathcal{F}$.

The set of degrees of freedom $d o f_{\mathcal{D}_{m}} \times d o f_{\mathcal{D}_{f}}$ (cf. Figure 3) is defined by

$$
\begin{aligned}
\operatorname{dof}_{\mathcal{D}_{m}} & =\mathcal{M} \cup\left(\bigcup_{\sigma \in \mathcal{F}} \overline{\mathcal{M}}_{\sigma}\right) \\
\operatorname{dof}_{\mathcal{D}_{f}} & =\mathcal{F}_{\Gamma} \cup \mathcal{E}_{\Gamma}, \\
\operatorname{dof}_{\text {Dirm }_{m}} & =\mathcal{F}_{\text {ext }}, \\
\operatorname{dof}_{\text {Dirf }_{f}} & =\mathcal{E}_{\Gamma} \cap \mathcal{E}_{\text {ext }},
\end{aligned}
$$

where for $\sigma \in \mathcal{F}$ and $K \in \mathcal{M}_{\sigma}$

$$
\bar{K}_{\sigma}= \begin{cases}\sigma & \text { if } \sigma \in \mathcal{F} \backslash \mathcal{F}_{\Gamma} \\ K_{\sigma} & \text { if } \sigma \in \mathcal{F}_{\Gamma}\end{cases}
$$

and $\overline{\mathcal{M}}_{\sigma}=\left\{\bar{K}_{\sigma} \mid K \in \mathcal{M}_{\sigma}\right\}$. We thus have

$$
\begin{aligned}
& X_{\mathcal{D}_{m}}=\left\{u_{K} \mid K \in \mathcal{M}\right\} \cup\left\{u_{\bar{K}_{\sigma}} \mid \sigma \in \mathcal{F}, \bar{K}_{\sigma} \in \overline{\mathcal{M}}_{\sigma}\right\}, \\
& X_{\mathcal{D}_{f}}=\left\{u_{\sigma} \mid \sigma \in \mathcal{F}_{\Gamma}\right\} \cup\left\{u_{e} \mid e \in \mathcal{E}_{\Gamma}\right\} .
\end{aligned}
$$

The discrete gradients in the matrix (respectively in the fracture domain) are piecewise constant in each 3D (respectively 2D) half diamond as shown at Figure 3. Following [15] we first define a cellwise constant discrete gradient

$$
\nabla_{K} u_{\mathcal{D}_{m}}=\frac{1}{|K|} \sum_{\sigma \in \mathcal{F}_{K}}|\sigma|\left(u_{\bar{K}_{\sigma}}-u_{K}\right) \mathbf{n}_{K, \sigma}
$$

which is exact on affine functions, but does not lead to a definite bilinear form (see [15], p. 8) and therefore has to be stabilized. Let

$$
\nabla_{K, \sigma} u_{\mathcal{D}_{m}}=\nabla_{K} u_{\mathcal{D}_{m}}+R_{K, \sigma}\left(u_{\mathcal{D}_{m}}\right) \mathbf{n}_{K, \sigma}, \sigma \in \mathcal{F}_{K},
$$

where $\mathbf{n}_{K, \sigma}$ is the unit normal vector of the face $\sigma \in \mathcal{F}_{K}$ outward to the cell $K$ and

$$
R_{K, \sigma}\left(u_{\mathcal{D}_{m}}\right)=\frac{\sqrt{d}}{d_{K, \sigma}}\left(u_{\bar{K}_{\sigma}}-u_{K}-\nabla_{K} u_{\mathcal{D}_{m}} \cdot\left(\mathbf{x}_{\sigma}-\mathbf{x}_{K}\right)\right)
$$

with $d_{K, \sigma}=\mathbf{n}_{K, \sigma} \cdot\left(\mathbf{x}_{\sigma}-\mathbf{x}_{K}\right)$. This leads to the definition of the matrix discrete gradient

$$
\nabla_{\mathcal{D}_{m}} u_{\mathcal{D}_{m}}(\mathbf{x})=\nabla_{K, \sigma} u_{\mathcal{D}_{m}} \text { on } D_{K, \sigma} \text { for all } K \in \mathcal{M}, \sigma \in \mathcal{F}_{K},
$$

where $D_{K, \sigma}=\bigcup_{e \in \mathcal{E}_{\sigma}} D_{K, \sigma, e}$ is the cone joining the face $\sigma$ to the cell center $\mathbf{x}_{K}$.

The fracture discrete gradient is defined analogously by

$$
\nabla_{\mathcal{D}_{f}} u_{\mathcal{D}_{f}}(\mathbf{x})=\nabla_{\sigma, e} u_{\mathcal{D}_{f}} \text { on } T_{\sigma, e} \text { for all } \sigma \in \mathcal{F}_{\Gamma}, e \in \mathcal{E}_{\sigma}
$$

with

$$
\nabla_{\sigma, e} u_{\mathcal{D}_{f}}=\nabla_{\sigma} u_{\mathcal{D}_{f}}+R_{\sigma, e}\left(u_{\mathcal{D}_{f}}\right) \mathbf{n}_{\sigma, e}
$$

and

$$
\nabla_{\sigma} u_{\mathcal{D}_{f}}=\frac{1}{|\sigma|} \sum_{e \in \mathcal{E}_{\sigma}}|e|\left(u_{e}-u_{\sigma}\right) \mathbf{n}_{\sigma, e}, \quad R_{\sigma, e}\left(u_{\mathcal{D}}\right)=\frac{\sqrt{d-1}}{d_{\sigma, e}}\left(u_{e}-u_{\sigma}-\nabla_{\sigma} u \cdot\left(\mathbf{x}_{e}-\mathbf{x}_{\sigma}\right)\right),
$$



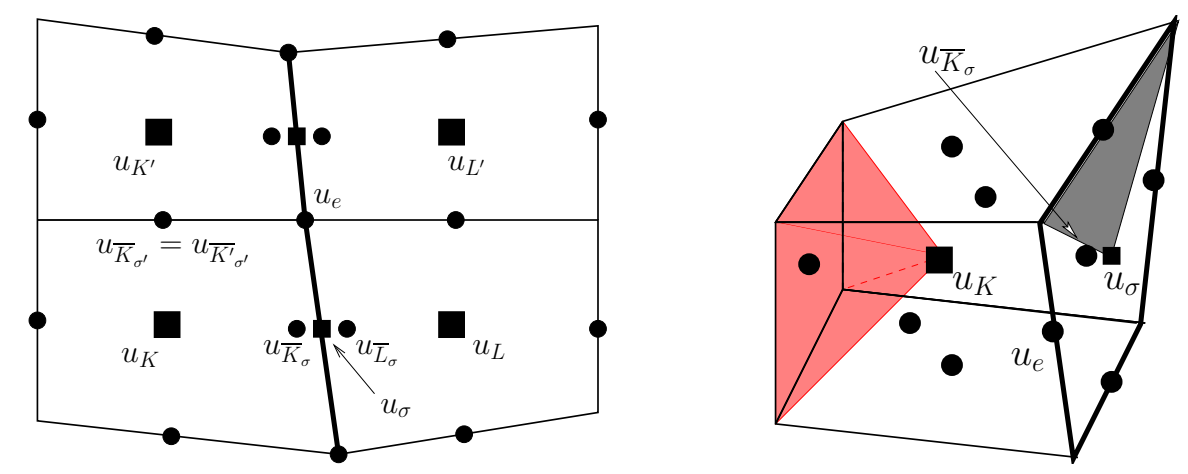

Figure 3: Left: Illustration of d.o.f. in 2D for four cells intersected by a vertical fracture (thick line).

Right: 3D cell $K$ touching a fracture face $\sigma$. Illustration of the polyhedron and polygone on which $\nabla_{\mathcal{D}_{m}}$ is constant (red) and $\nabla_{\mathcal{D}_{f}}$ is constant (grey).

where $\mathbf{n}_{\sigma, e}$ is the unit normal vector to the edge $e$ in the tangent plane of the face $\sigma$ and outward to the face $\sigma, d_{\sigma, e}=\mathbf{n}_{\sigma, e} \cdot\left(\mathbf{x}_{e}-\mathbf{x}_{\sigma}\right)$, and $T_{\sigma, e}$ is the triangle of base $e$ and vertex $\mathbf{x}_{\sigma}$.

The function reconstruction operators are piecewise constant on a partition of the cells and of the fracture faces. For $K \in \mathcal{M}$ let $d o f_{K}=\left\{\bar{K}_{\sigma}, \sigma \in \mathcal{F}_{K}\right\}$. Analogously, in the fracture domain, for $\sigma \in \mathcal{F}_{\Gamma}$ let $d o f_{\sigma}=\mathcal{E}_{\sigma}$. We introduce, for any given $K \in \mathcal{M}$, a partition $\left\{\omega_{K}^{\nu}\right\}_{\nu \in\{K\} \cup d o f_{K} \backslash d o f_{\text {Dir }}}$

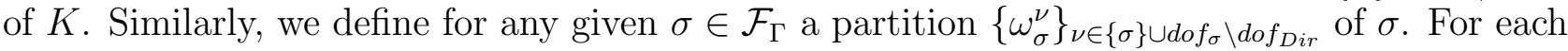
$\nu \in d o f_{\mathcal{D}_{m}}$, we define the open set $\omega_{\nu}=\operatorname{int}\left(\bigcup_{K \in \mathcal{M}} \bar{\omega}_{K}^{\nu}\right)$, with the convention $\omega_{K}^{\nu}=\emptyset$, if $\nu \notin d o f_{K}$. For each $\nu \in d o f_{\mathcal{D}_{f}}$, we define the open set $\omega_{\nu}=\operatorname{int}\left(\bigcup_{\sigma \in \mathcal{F}} \bar{\omega}_{\sigma}^{\nu}\right)$, where $\omega_{\sigma}^{\nu}=\emptyset$, if

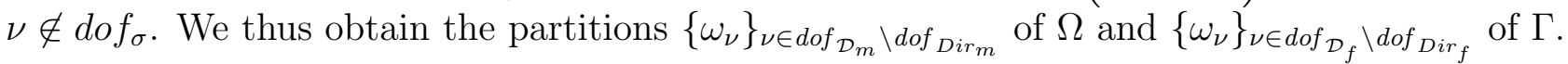
Like the VAG-CV discretization, the HFV discretization is particularly adapted for the treatment of multiphase flow processes through heterogeneous media (cf. [16]). Provided the mesh is comform w.r.t. the heterogeneous layers, we can assume that key geological quantities are constant per cell and per fracture face. Therefore, in the numerical scheme, we do not need to reconstruct the just introduced partitions explicitly, but only have to define their corresponding volumes. Finally, we need a mapping between the degrees of freedom of the matrix domain, which are situated on one side of the fracture network, and the set of indices $\chi$. For $\sigma \in \mathcal{F}_{\Gamma}$ and $\bar{K}_{\sigma} \in \overline{\mathcal{M}}_{\sigma}$ holds by definition $\bar{K}_{\sigma}=\{K\}$ for a $K \in \mathcal{M}_{\sigma}$ and hence $\mathbf{n}_{\bar{K}_{\sigma}}=\mathbf{n}_{K, \sigma}$ is well defined. We obtain the one-element set $\chi\left(\bar{K}_{\sigma}\right)=\left\{\alpha \in \chi \mid \mathbf{n}_{\bar{K}_{\sigma}}=\mathbf{n}_{\alpha}\right.$ on $\left.\sigma\right\}$ and therefore the notation $\alpha\left(\bar{K}_{\sigma}\right)=\alpha \in \chi\left(\bar{K}_{\sigma}\right)$.

We define the HFV scheme's reconstruction operators by

$$
\begin{aligned}
& \text { - } \Pi_{\mathcal{D}_{m}} u_{\mathcal{D}_{m}}=\sum_{\nu \in \operatorname{dof}_{\mathcal{D}_{m}} \backslash \operatorname{dof}_{D_{i r_{m}}}} u_{\nu} \mathbb{1}_{\omega_{\nu}}, \\
& \text { - } \Pi_{\mathcal{D}_{f}} u_{\mathcal{D}_{f}}=\sum_{\nu \in \operatorname{dof}_{\mathcal{D}_{f}} \backslash d o f_{D_{i r_{f}}}} u_{\nu} \mathbb{1}_{\omega_{\nu}}, \\
& \text { - } \llbracket u_{\mathcal{D}} \rrbracket_{\mathcal{D}}^{\alpha}=\sum_{\sigma \in \mathcal{F}_{\Gamma}} \sum_{\bar{K}_{\sigma} \in \overline{\mathcal{M}}_{\sigma}}\left(u_{\bar{K}_{\sigma}}-u_{\sigma}\right) \delta_{\alpha\left(\bar{K}_{\sigma}\right) \alpha} \mathbb{1}_{\sigma} \quad \text { for all } \alpha \in \chi .
\end{aligned}
$$

Proposition 4.3 Let us consider a sequence of meshes $\left(\mathcal{M}^{l}\right)_{l \in \mathbb{N}}$ and let us assume that the sequence $\left(\mathcal{T}^{l}\right)_{l \in \mathbb{N}}$ of tetrahedral submeshes is shape regular, i.e. $\theta_{\mathcal{T}^{l}}$ is uniformly bounded. We 
also assume that $\lim _{l \rightarrow \infty} h_{\mathcal{T}^{l}}=0$. Then, any corresponding sequence of gradient discretizations $\left(\mathcal{D}^{l}\right)_{l \in \mathbb{N}}$, defined by (4.8), (4.9), (4.10) and definition (4.11), is coercive, consistent and limit conforming.

Proof Let us first give the proof of the proposition in the special case $\omega_{\bar{K}_{\sigma}}=\omega_{e}=\emptyset$ for all $\bar{K}_{\sigma} \in \bigcup_{\sigma \in \mathcal{F}} \overline{\mathcal{M}}_{\sigma}$ and $e \in \mathcal{E}_{\Gamma}$. We denoting in that case by $\Pi_{\mathcal{M}}$ and $\Pi_{\mathcal{F}}$ the HFV matrix and fracture function reconstruction operators.

Coercivity: We first prove that limit conformity against regular test functions, as proved below, implies coercivity.

Assume that the sequence of discretizations $\left(\mathcal{D}^{l}\right)_{l \in \mathbb{N}}$ is not coercive. Then we can find a sequence $\left(\left(u_{\mathcal{D}_{m}^{l}}, u_{\mathcal{D}_{f}^{l}}\right)\right)_{l \in \mathbb{N}}$ with $\left(u_{\mathcal{D}_{m}^{l}}, u_{\mathcal{D}_{f}^{l}}\right) \in X_{\mathcal{D}^{l}}^{0}$, such that

$$
\left\|\Pi_{\mathcal{D}_{m}^{l}} u_{\mathcal{D}_{m}^{l}}\right\|_{L^{2}(\Omega)}+\left\|\Pi_{\mathcal{D}_{f}^{l}} u_{\mathcal{D}_{f}^{l}}\right\|_{L^{2}(\Gamma)}=1 \quad \text { and } \quad\left\|\left(u_{\mathcal{D}_{m}^{l}}, u_{\mathcal{D}_{f}^{l}}\right)\right\|_{\mathcal{D}^{l}}<\frac{1}{l} .
$$

Then, it follows from a compactness result of [11] that there exists a $u=\left(u_{m}, u_{f}\right) \in L^{2}(\Omega) \times$ $L^{2}(\Gamma)$, s.t. up to a subsequence

$$
\left(\Pi_{\mathcal{D}_{m}^{l}} u_{\mathcal{D}_{m}^{l}}, \Pi_{\mathcal{D}_{f}^{l}} u_{\mathcal{D}_{f}^{l}}\right) \longrightarrow\left(u_{m}, u_{f}\right) \quad \text { in } L^{2}(\Omega) \times L^{2}(\Gamma) \quad(\text { for } l \rightarrow \infty)
$$

and therefore $\left\|u_{m}\right\|_{L^{2}(\Omega)}+\left\|u_{f}\right\|_{L^{2}(\Gamma)}=1$. On the other hand it follows from the discretization's limit conformity against regular test functions stated in Corollary 3.1 that $\left(u_{m}, u_{f}\right) \in V^{0}$ and that up to a subsequence

$$
\left\{\begin{array}{l}
\nabla_{\mathcal{D}_{m}} u_{\mathcal{D}_{m}^{l}} \rightarrow \nabla u_{m} \quad \text { in } L^{2}(\Omega)^{d}, \\
\nabla_{\mathcal{D}_{f}} u_{\mathcal{D}_{f}^{l}} \rightarrow \nabla_{\tau} u_{f} \quad \text { in } L^{2}(\Gamma)^{d-1}, \\
\llbracket u_{\mathcal{D}} \rrbracket_{\mathcal{D}}^{\alpha} \rightarrow \llbracket v \rrbracket^{\alpha} \quad \text { in } L^{2}\left(\Gamma_{\alpha}\right), \text { for } \alpha \in \chi .
\end{array}\right.
$$

Since by construction $\left\|\left(u_{\mathcal{D}_{m}^{l}}, u_{\mathcal{D}_{f}^{l}}\right)\right\|_{\mathcal{D}^{l}} \rightarrow 0$ holds, we obtain $\left\|\left(u_{m}, u_{f}\right)\right\|_{V^{0}}=0$. But $\|\cdot\|_{V^{0}}$ is a norm on $V^{0}$, which contradicts the fact that $\left\|u_{m}\right\|_{L^{2}(\Omega)}+\left\|u_{f}\right\|_{L^{2}(\Gamma)}=1$.

Consistency: For $\left(\varphi_{m}, \varphi_{f}\right) \in C_{\Omega}^{\infty} \times C_{\Gamma}^{\infty}$ let us define the projection $P_{\mathcal{D}_{m}} \varphi_{m} \in X_{\mathcal{D}_{m}}^{0}$ such that $\left(P_{\mathcal{D}_{m}} \varphi_{m}\right)_{\nu}=\varphi_{m}\left(\mathbf{x}_{\nu}\right)$ for all $\nu \in d o f_{\mathcal{D}_{m}}$, and the projection $P_{\mathcal{D}_{f}} \varphi_{f} \in X_{\mathcal{D}_{f}}^{0}$ such that $\left(P_{\mathcal{D}_{f}} \varphi_{f}\right)_{\nu}=\varphi_{f}\left(\mathbf{x}_{\nu}\right)$ for all $\nu \in d o f_{\mathcal{D}_{f}}$. It is easily seen that for $u_{\mathcal{D}}=\left(P_{\mathcal{D}_{m}} \varphi_{m}, P_{\mathcal{D}_{f}} \varphi_{f}\right)$ holds

$$
\begin{aligned}
& \left\|\Pi_{\mathcal{M}} u_{\mathcal{D}_{m}}-\varphi_{m}\right\|_{L^{2}(\Omega)}+\left\|\Pi_{\mathcal{F}} u_{\mathcal{D}_{f}}-\varphi_{f}\right\|_{L^{2}(\Gamma)} \\
& +\sum_{\alpha \in \chi}\left\|\llbracket u_{\mathcal{D}} \rrbracket_{\mathcal{D}}^{\alpha}-\llbracket u \rrbracket^{\alpha}\right\|_{L^{2}\left(\Gamma_{\alpha}\right)} \leq c s t \cdot h_{\mathcal{T}} \cdot\left(|\Gamma|^{\frac{1}{2}}+|\Omega|^{\frac{1}{2}}\right),
\end{aligned}
$$

with a constant depending only on $\varphi$. Furthermore, it follows from Lemma 4.3 of [15] that there exists $C>0$ depending only on $\theta_{\mathcal{T}}$ and $\varphi$ such that

$$
\left\|\nabla_{\mathcal{D}_{m}} u_{\mathcal{D}_{m}}-\nabla \varphi\right\|_{L^{2}(\Omega)}+\left\|\nabla_{\mathcal{D}_{f}} u_{\mathcal{D}_{f}}-\nabla \varphi\right\|_{L^{2}(\Gamma)} \leq C h_{\mathcal{T}}
$$

Taking into account that $C_{\Omega}^{\infty} \times C_{\Gamma}^{\infty}$ is dense in $V^{0}$, we see that the treated discretisation is consistent.

Limit Conformity: In [7], proof of Proposition 10, the limit conformity of HFV scheme is shown in the case of continuous pressure at the matrix-fracture interfaces. This proof adapts straightforward to our case, so that we obtain

$$
w_{\mathcal{D}}\left(u_{\mathcal{D}}, \mathbf{q}\right) \leq C \cdot h_{\mathcal{T}} \cdot\left\|u_{\mathcal{D}}\right\|_{\mathcal{D}} \quad \text { for all } \mathbf{q} \in C_{W_{m}}^{\infty} \times C_{W_{f}}^{\infty}
$$


This result is shown above to imply coercivity. From the coercivity and the density of $C_{W_{m}}^{\infty} \times C_{W_{f}}^{\infty}$ in $W$, we derive limit conformity on the whole space of test functions.

Generalization to arbitrary HFV discretizations: Let us notice, that from [15] Lemma 4.1 follows that there are positive constants $C_{m}$ and $C_{f}$ only depending on $\theta_{\mathcal{T}}$, such that for all $u_{\mathcal{D}} \in X_{\mathcal{D}}$ holds

$$
\begin{array}{r}
\left\|\Pi_{\mathcal{M}} u_{\mathcal{D}_{m}}-\Pi_{\mathcal{D}_{m}} u_{\mathcal{D}_{m}}\right\|_{L^{2}(\Omega)}^{2}=\sum_{K \in \mathcal{M}} \sum_{\sigma \in \mathcal{F}_{K}}\left|\omega_{K}^{\bar{K}_{\sigma}}\right|\left(u_{K}-u_{\bar{K}_{\sigma}}\right)^{2} \leq C_{m} \cdot h_{\mathcal{T}}^{2} \cdot\left\|\nabla_{\mathcal{D}_{m}} u_{\mathcal{D}_{m}}\right\|_{L^{2}(\Omega)^{d}}^{2} \\
\left\|\Pi_{\mathcal{F}} u_{\mathcal{D}_{f}}-\Pi_{\mathcal{D}_{f}} u_{\mathcal{D}_{f}}\right\|_{L^{2}(\Gamma)}^{2}=\sum_{\sigma \in \mathcal{F}_{\Gamma}} \sum_{e \in \mathcal{E}_{\sigma}}\left|\omega_{\sigma}^{e}\right|\left(u_{\sigma}-u_{e}\right)^{2} \leq C_{f} \cdot h_{\Delta}^{2} \cdot\left\|\nabla_{\mathcal{D}_{f}} u_{\mathcal{D}_{f}}\right\|_{L^{2}(\Gamma)^{d-1}}^{2} .
\end{array}
$$

It follows that coercivity, consistency and limit conformity can immediately be shown.

Remark 4.3 The precedent proof shows that for solutions $\left(u_{m}, u_{f}\right) \in V^{0}$ and $\left(\mathbf{q}_{m}, \mathbf{q}_{f}\right) \in W$ of (2.1) such that $u_{m} \in C^{2}(\bar{K}), u_{f} \in C^{2}(\bar{\sigma}), \mathbf{q}_{m} \in\left(C^{1}(\bar{K})\right)^{d}, \mathbf{q}_{f} \in\left(C^{1}(\bar{\sigma})\right)^{d-1}$ for all $K \in \mathcal{M}$ and all $\sigma \in \Gamma_{f}$, the HFV schemes are consistent and limit conforming of order 1, and therefore convergent of order 1.

\section{$5 \quad$ Numerical Results}

The objective of this section is to compare the VAG-FE, VAG-CV, and the HFV schemes in terms of accuracy and CPU efficiency for both hexahedral and tetrahedral meshes on heterogeneous isotropic and anisotropic media. For that purpose an original family of analytical solutions is built for the fixed value of the parameter $\xi=1$. We refer to [21], [4], [2] for a comparison of the solutions obtained with different values of the parameter $\xi \in\left[\frac{1}{2}, 1\right]$ with the solution obtained with a 3D representation of the fractures.

Table 1 exhibits for the hexahedral and tetrahedral meshes, as well as for both the VAG and HFV schemes, the number of degrees of freedom ( $\mathrm{Nb}$ dof), the number of d.o.f. after elimination of the cell and Dirichlet unknowns (nb dof el.), and the number of nonzero element in the linear system after elimination without any fill-in of the cell unknowns ( $\mathrm{Nb} \mathrm{Jac).}$

The hexahedral meshes used here are divided into two families. The first family consists of Cartesian meshes and hence satisfies the well known ortogonality property. The second family of meshes is obtained from the previous one by a perturbation of its nodes. The perturbation is chosen randomly inside the ball around each node of radius one third the distance to it's closest neighbour, of course in respect of the domain's boundaries. The perturbation of a fracture node is done in the fracture plane. Note that this leads to hexahedral cells with non planar faces and therefore the HFV scheme is no longer consistent on this family of meshes.

In all test cases, the linear system obtained after elimination of the cell unknowns is solved using GMRes iterative solver with the stopping criteria $10^{-10}$. The GMRes solver is preconditioned by ILUT [24], [25] using the thresholding parameter $10^{-4}$ chosen small enough in such a way that all the linear systems can be solved for both schemes and for all meshes. In tables 2 and 3, we report the number of GMRes iterations Iter and the CPU time taking into account the elimination of the cell unknowns, the ILUT factorization, the GMRes iterations, and the computation of the cell values. Regarding the choice of the preconditioner, we also mention that the resolution of the anisotropic problem with an AMG preconditioner revealed less efficiency compared to ILUT on tetrahedral meshes (particularly for the HFV scheme).

We ran the program on a 2,6 GHz Intel Core i5 processor with $8 \mathrm{~GB} 1600 \mathrm{MHz}$ DDR3 memory. 


\subsection{A class of analytical solutions}

We consider a 3-dimensional domain $\Omega=(-0.5,0.5)^{3}$ with four intersecting fractures $\Gamma_{12}=$ $\{(x, y, z) \in \Omega \mid x=0, y>0\}, \Gamma_{23}=\{(x, y, z) \in \Omega \mid y=0, x>0\}, \Gamma_{34}=\{(x, y, z) \in \Omega \mid$ $x=0, y<0\}$ and $\Gamma_{14}=\{(x, y, z) \in \Omega \mid y=0, x<0\}$. We also introduce four disjoint subsets of $\Omega, \Omega_{1}=\{(x, y, z) \in \Omega \mid y>0, x<0\}, \Omega_{2}=\{(x, y, z) \in \Omega \mid y>0, x>0\}$, $\Omega_{3}=\{(x, y, z) \in \Omega \mid y<0, x>0\}$ and $\Omega_{4}=\{(x, y, z) \in \Omega \mid y<0, x<0\}$.

Derivation: For $\left(u_{m}, u_{f}\right) \in V$, we denote $u_{m}(x, y, z)=u_{i}(x, y, z)$ on $\Omega_{i}, i=1, \ldots, 4$ and $u_{f}(x, y, z)=u_{i j}(y, z)$ on $\Gamma_{i j}, i j \in J$, where we have introduced $J=\{12,23,34,14\}$. We assume that a solution of the discontinuous pressure model writes in the fracture network as $u_{i j}(y, z)=$ $\alpha_{f}(z)+\beta_{i j}(z) \gamma_{i j}(y), i j \in J$ and in the matrix domain as

$$
\left\{\begin{array}{l}
u_{1}(x, y, z)=\alpha_{1}(z) u_{12}(y, z) u_{14}(x, z) \\
u_{2}(x, y, z)=\alpha_{2}(z) u_{12}(y, z) u_{23}(x, z) \\
u_{3}(x, y, z)=\alpha_{3}(z) u_{34}(y, z) u_{23}(x, z) \\
u_{4}(x, y, z)=\alpha_{4}(z) u_{34}(y, z) u_{14}(x, z)
\end{array}\right.
$$

We assume $\gamma_{i j}(0)=0, i j \in J$ so that the continuity of $u_{f}$ at the fracture-fracture intersection is satisfied and we set $\gamma_{i j}^{\prime}(0)=1$, to ease the following calculations. For $i=1, \ldots, 4$ let $K_{i}=\Lambda_{m}\left\lceil_{\Omega_{i}}\right.$ and for $i j \in J$ let $T_{i j}=T_{f} \uparrow_{\Gamma_{i j}}$. From the conditions $\gamma_{\mathbf{n}, \alpha} \mathbf{q}_{m}=T_{f} \llbracket u \rrbracket^{\alpha}$ on $\Gamma_{\alpha}, \alpha \in \chi$, we then get, after some effort in computation,

$$
\begin{aligned}
\alpha_{1}(z) & =\left(\alpha_{f}(z)-\frac{K_{1 y}}{T_{14}} \beta_{12}(z)\right)^{-1}, & & \alpha_{2}(z)=\left(\alpha_{f}(z)-\frac{K_{1 y} K_{2 x} K_{3 y} K_{4 x}}{K_{1 x} K_{3 x} K_{4 y} T_{23}} \beta_{12}(z)\right)^{-1}, \\
\alpha_{3}(z) & =\left(\alpha_{f}(z)-\frac{K_{1 y} K_{3 y} K_{4 x} T_{12}}{K_{1 x} K_{4 y} T_{23} T_{34}} \beta_{12}(z)\right)^{-1}, & & \alpha_{4}(z)=\left(\alpha_{f}(z)-\frac{K_{1 y} K_{4 x} T_{12}}{K_{1 x} T_{14} T_{34}} \beta_{12}(z)\right)^{-1}, \\
\beta_{23}(z) & =\frac{K_{1 y} K_{3 y} K_{4 x} T_{12}}{K_{1 x} K_{3 x} K_{4 y} T_{23}} \beta_{12}(z), & & \beta_{34}(z)=-\frac{K_{1 y} K_{4 x} T_{12}}{K_{1 x} K_{4 y} T_{34}} \beta_{12}(z) \\
\beta_{14}(z) & =-\frac{K_{1 y} T_{12}}{K_{1 x} T_{14}} \beta_{12}(z), & & \frac{K_{1 y} K_{2 x} K_{3 y} K_{4 x}}{K_{1 x} K_{2 y} K_{3 x} K_{4 y}}=1 .
\end{aligned}
$$

Obviously, we have taken $\alpha_{f}$ and $\beta_{12}$ as degrees of freedom, here. However, these functions must be chosen in such a way that $\frac{1}{\alpha_{i}(z)} \neq 0$ for $i=1, \ldots, 4$.

Remark 5.1 We would like to explicitly calculate the jump at the matrix-fracture interfaces for this class of solutions. At $\Gamma_{i j}$ we have

$$
\begin{array}{ll}
u_{i}(0, y, z)-u_{j}(0, y, z)=\left(\alpha_{i}(z)-\alpha_{j}(z)\right) \cdot \alpha_{f}(z) \cdot u_{i j}(y, z), & \text { for } i j \in\{12,34\} \\
u_{i}(x, 0, z)-u_{j}(x, 0, z)=\left(\alpha_{i}(z)-\alpha_{j}(z)\right) \cdot \alpha_{f}(z) \cdot u_{i j}(x, z), & \text { for } i j \in\{23,14\} .
\end{array}
$$

From (5.1), we observe, that the pressure becomes continuous at the matrix-fracture interfaces, as the $T_{i j}$ tend to $\infty$ uniformly.

Remark 5.2 In order to obtain solutions with discontinuities at the matrix-fracture interfaces, we had to omit the constraint of flux conservation at fracture-fracture intersections. Numerically this is treated by adding an additional source term for the corresponding fracture unknowns.

\subsection{Test Case}

We define a solution by setting $\alpha_{f}(z)=e^{\sin (\pi z)}, \beta_{12}(z)=-1, \gamma_{12}(y)=\cos (2 \pi y)+y-1$, $\gamma_{23}(x)=x, \gamma_{34}(y)=-e^{\cos (\pi y)}+y+e, \gamma_{14}(x)=\frac{\sin (\pi x)}{\pi}$. The parameters we used for the different test cases are 
- Isotropic Heterogeneous Permeability:

$$
\begin{aligned}
K_{1 x} & =K_{1 y}=K_{1 z}=1, K_{2 x}=K_{2 y}=K_{2 z}=100 \\
K_{3 x} & =K_{3 y}=K_{3 z}=3, K_{4 x}=K_{4 y}=K_{4 z}=40 \\
T_{12} & =1, T_{23}=0.2, T_{34}=100, T_{14}=10 \\
K_{12} & =1, K_{23}=2, K_{34}=3, K_{14}=10 .
\end{aligned}
$$

- Anisotropic Heterogeneous Permeability:

$$
\begin{aligned}
K_{1 x} & =K_{1 z}=1, K_{1 y}=50, K_{2 x}=K_{2 z}=2, K_{2 y}=100, \\
K_{3 y} & =K_{3 z}=3, K_{3 x}=30, K_{4 z}=4, K_{4 x}=K_{4 y}=40, \\
T_{12} & =T_{23}=T_{34}=T_{14}=1, \\
K_{12} & =K_{23}=K_{34}=K_{14}=1 .
\end{aligned}
$$

In Figures 5.2 we represent the normalized $L^{2}$ norms of the errors, which are calculated as follows:

- normalized error of the solution: $\operatorname{err}_{\text {sol }}=\frac{\left\|\Pi_{\mathcal{D}_{m}} u_{\mathcal{D}_{m}}-u_{m}\right\|_{L^{2}(\Omega)}+\left\|\Pi_{\mathcal{D}_{f}} u_{\mathcal{D}_{f}}-u_{f}\right\|_{L^{2}(\Gamma)}}{\left\|u_{m}\right\|_{L^{2}(\Omega)}+\left\|u_{f}\right\|_{L^{2}(\Gamma)}}$

- normalized error of the gradient: $\operatorname{err}_{\text {grad }}=\frac{\left\|\nabla_{\mathcal{D}_{m}} u_{\mathcal{D}_{m}}-\nabla u_{m}\right\|_{L^{2}(\Omega)}+\left\|\nabla_{\mathcal{D}_{f}} u_{\mathcal{D}_{f}}-\nabla_{\tau} u_{f}\right\|_{L^{2}(\Gamma)}{ }^{d-1}}{\left\|\nabla u_{m}\right\|_{L^{2}(\Omega)^{d}}+\left\|\nabla \tau_{\tau_{f}}\right\|_{L^{2}(\Gamma)^{d-1}}}$

In the following tables is additionally found the normalized error of the jump: $\operatorname{err}_{j u m p}=\frac{\sum_{\alpha \in \chi}\left\|\llbracket u_{\mathcal{D}} \rrbracket_{\mathcal{D}}^{\alpha}-\llbracket u \rrbracket^{\alpha}\right\|_{L^{2}\left(\Gamma_{\alpha}\right)}}{\sum_{\alpha \in \chi}\left\|\llbracket u \rrbracket^{\alpha}\right\|_{L^{2}\left(\Gamma_{\alpha}\right)}}$.

\begin{tabular}{|c|c|c|c|c|c|c|c|}
\cline { 2 - 7 } \multicolumn{2}{|c|}{ KAG } & \multicolumn{3}{c|}{ HF V } \\
\hline Key & Nb Cells & Nb dof & Nb dof el. & Nb Jac & Nb dof & Nb dof el. & Nb Jac \\
\hline Hexahedral Meshes \\
\hline 1 & 512 & 1949 & 1437 & 31253 & 2776 & 2264 & 20696 \\
\hline 2 & 4096 & 11701 & 7605 & 178845 & 19248 & 15152 & 150320 \\
\hline 3 & 32768 & 79205 & 46437 & 1154861 & 142432 & 109664 & 1141856 \\
\hline 4 & 262144 & 578245 & 316101 & 8152653 & 1093824 & 831680 & 8892608 \\
\hline 5 & 2097152 & 4408709 & 2311557 & 60910733 & 8569216 & 6472064 & 70173056 \\
\hline Tetrahedral Meshes & \multicolumn{7}{|l|}{} \\
\hline 6 & 1337 & 2514 & 1177 & 18729 & 4943 & 3606 & 22642 \\
\hline 7 & 10706 & 15765 & 5059 & 81741 & 35520 & 24814 & 164246 \\
\hline 8 & 100782 & 131204 & 30422 & 492158 & 317367 & 216585 & 1474817 \\
\hline 9 & 220106 & 279281 & 59175 & 956659 & 685718 & 465612 & 3190244 \\
\hline 10 & 428538 & 533442 & 104904 & 1694008 & 1324614 & 896076 & 6167300 \\
\hline 11 & 2027449 & 2452416 & 424967 & 6818299 & 6193783 & 4166334 & 28862986 \\
\hline
\end{tabular}

Table 1: Key defines the mesh reference; $\mathbf{N b}$ Cells is the number of cells of the mesh; $\mathbf{N b}$ dof is the number of discrete unknowns; $\mathbf{N b}$ dof el. is the number of discrete unknowns after elimination of cell unknowns; Nb Jac refers to the number of non-zero Jacobian entries after elimination of the cell unknowns and equations. 

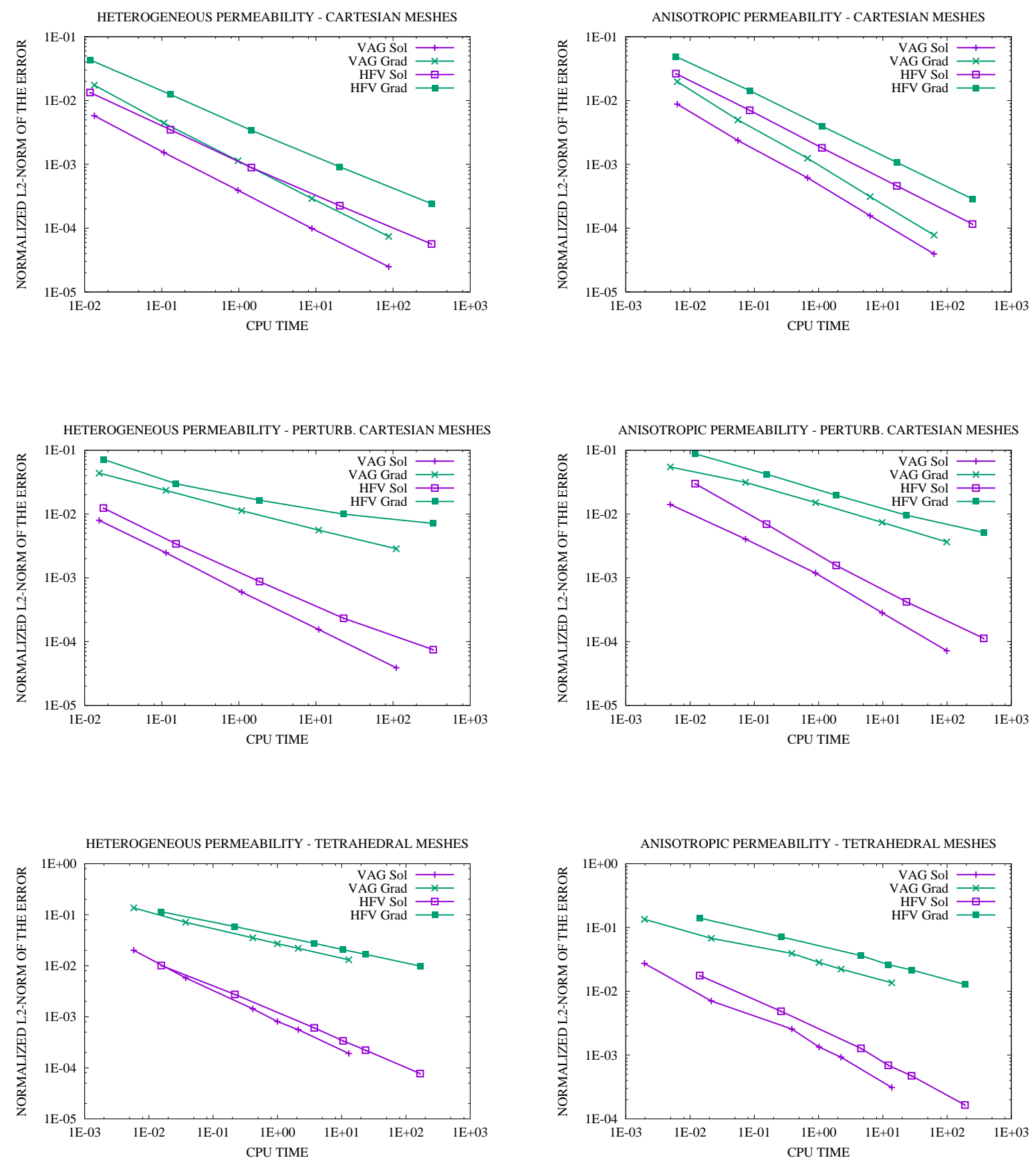

Figure 4: Comparison of VAG-FE and HFV on Cartesian (upper line), perturbated Cartesian (mid line) and tetrahedral (lower line) meshes. Left column: heterogeneous permeability. Right column: heterogeneous and anisotropic permeability. 


\begin{tabular}{|c|c|c|c|c|c|c|c|c|}
\hline & \multicolumn{8}{|c|}{ Heterogeneous Permeability: $V A G$} \\
\hline Key & Iter & CPU & $e r r_{s o l}$ & err grad & $e r r_{\text {jump }}$ & $\alpha_{\text {sol }}$ & $\alpha_{\text {grad }}$ & $\alpha_{\text {jump }}$ \\
\hline \multicolumn{9}{|c|}{ Hexahedral Meshes (Cartesian) } \\
\hline 1 & 8 & $1.34 \mathrm{E}-2$ & $5.78 \mathrm{E}-3$ & $1.74 \mathrm{E}-2$ & 8.99E-3 & - & - & - \\
\hline 2 & 12 & 0.11 & $1.53 \mathrm{E}-3$ & $4.44 \mathrm{E}-3$ & $2.53 \mathrm{E}-3$ & 1.92 & 1.97 & 1.83 \\
\hline 3 & 22 & 0.98 & $3.92 \mathrm{E}-4$ & $1.14 \mathrm{E}-3$ & $6.72 \mathrm{E}-4$ & 1.97 & 1.96 & 1.91 \\
\hline 4 & 41 & 8.86 & $9.89 \mathrm{E}-5$ & $2.91 \mathrm{E}-4$ & $1.73 \mathrm{E}-4$ & 1.99 & 1.97 & 1.96 \\
\hline 5 & 79 & 87.91 & $2.48 \mathrm{E}-5$ & $7.40 \mathrm{E}-5$ & $4.40 \mathrm{E}-5$ & 1.99 & 1.98 & 1.98 \\
\hline \multicolumn{9}{|c|}{ Hexahedral Meshes (perturbated Cartesian) } \\
\hline 1 & 8 & $1.54 \mathrm{E}-2$ & $7.96 \mathrm{E}-3$ & $4.36 \mathrm{E}-2$ & $1.14 \mathrm{E}-2$ & - & - & - \\
\hline 2 & 12 & 0.11 & $2.46 \mathrm{E}-3$ & $2.34 \mathrm{E}-2$ & $3.62 \mathrm{E}-3$ & 1.69 & 0.89 & 1.65 \\
\hline 3 & 21 & 1.09 & $5.99 \mathrm{E}-4$ & $1.12 \mathrm{E}-2$ & $8.92 \mathrm{E}-4$ & 2.04 & 1.05 & 2.02 \\
\hline 4 & 41 & 10.84 & $1.55 \mathrm{E}-4$ & $5.58 \mathrm{E}-3$ & $2.32 \mathrm{E}-4$ & 1.95 & 1.01 & 1.94 \\
\hline 5 & 77 & 110.13 & $3.89 \mathrm{E}-5$ & $2.85 \mathrm{E}-3$ & $5.872 \mathrm{E}-5$ & 1.99 & 0.96 & 1.98 \\
\hline \multicolumn{9}{|c|}{ Tetrahedral Meshes } \\
\hline 6 & 7 & $5.82 \mathrm{E}-3$ & $2.01 \mathrm{E}-2$ & 0.14 & $2.25 \mathrm{E}-2$ & - & - & - \\
\hline 7 & 10 & $3.73 \mathrm{E}-2$ & $5.78 \mathrm{E}-3$ & 7.09E-2 & $7.03 \mathrm{E}-3$ & 1.80 & 0.94 & 1.68 \\
\hline 8 & 20 & 0.41 & $1.44 \mathrm{E}-3$ & $3.52 \mathrm{E}-2$ & $1.81 \mathrm{E}-3$ & 1.86 & 0.94 & 1.82 \\
\hline 9 & 26 & 1.00 & $8.11 \mathrm{E}-4$ & $2.71 \mathrm{E}-2$ & $1.06 \mathrm{E}-3$ & 2.20 & 1.01 & 2.06 \\
\hline 10 & 32 & 2.11 & $5.60 \mathrm{E}-4$ & $2.19 \mathrm{E}-2$ & $7.36 \mathrm{E}-4$ & 1.67 & 0.95 & 1.62 \\
\hline 11 & 53 & 12.92 & $1.92 \mathrm{E}-4$ & $1.31 \mathrm{E}-2$ & $2.58 \mathrm{E}-4$ & 2.07 & 1.00 & 2.03 \\
\hline
\end{tabular}

\begin{tabular}{|c|c|c|c|c|c|c|c|c|}
\hline & \multicolumn{8}{|c|}{ Heterogeneous Permeability: $\boldsymbol{H F V}$} \\
\hline Key & Iter & CPU & $e r r_{\text {sol }}$ & err $r_{\text {grad }}$ & $e r r_{j u m p}$ & $\alpha_{\text {sol }}$ & $\alpha_{\text {grad }}$ & $\alpha_{\text {jump }}$ \\
\hline \multicolumn{9}{|c|}{ Hexahedral Meshes (Cartesian) } \\
\hline 1 & 11 & $1.18 \mathrm{E}-2$ & $1.34 \mathrm{E}-2$ & $4.3 \mathrm{E}-2$ & $2.15 \mathrm{E}-2$ & - & - & - \\
\hline 2 & 19 & 0.13 & $3.49 \mathrm{E}-3$ & $1.24 \mathrm{E}-2$ & $5.44 \mathrm{E}-3$ & 1.94 & 1.80 & 1.98 \\
\hline 3 & 35 & 1.45 & $8.91 \mathrm{E}-4$ & $3.41 \mathrm{E}-3$ & $1.38 \mathrm{E}-3$ & 1.97 & 1.86 & 1.98 \\
\hline 4 & 73 & 20.36 & $2.25 \mathrm{E}-4$ & $9.15 \mathrm{E}-4$ & $3.47 \mathrm{E}-4$ & 1.99 & 1.90 & 1.99 \\
\hline 5 & 141 & 315.38 & $5.65 \mathrm{E}-5$ & $2.42 \mathrm{E}-4$ & $8.69 \mathrm{E}-5$ & 1.99 & 1.92 & 2.00 \\
\hline \multicolumn{9}{|c|}{ Hexahedral Meshes (perturbated Cartesian) } \\
\hline 1 & 10 & $1.74 \mathrm{E}-2$ & $1.23 \mathrm{E}-2$ & $7.17 \mathrm{E}-2$ & $2.19 \mathrm{E}-2$ & - & - & - \\
\hline 2 & 18 & 0.15 & $3.40 \mathrm{E}-3$ & $2.97 \mathrm{E}-2$ & $5.74 \mathrm{E}-3$ & 1.86 & 1.27 & 1.93 \\
\hline 3 & 33 & 1.85 & $8.73 \mathrm{E}-4$ & $1.64 \mathrm{E}-2$ & $1.67 \mathrm{E}-3$ & 1.96 & 0.85 & 1.77 \\
\hline 4 & 69 & 22.83 & $2.32 \mathrm{E}-4$ & $9.94 \mathrm{E}-3$ & $5.44 \mathrm{E}-4$ & 1.90 & 0.72 & 1.62 \\
\hline 5 & 128 & 330.77 & $7.48 \mathrm{E}-5$ & $7.12 \mathrm{E}-3$ & $2.36 \mathrm{E}-4$ & 1.63 & 0.48 & 1.19 \\
\hline \multicolumn{9}{|c|}{ Tetrahedral Meshes } \\
\hline 6 & 12 & $1.56 \mathrm{E}-2$ & $1.01 \mathrm{E}-2$ & 0.11 & $1.74 \mathrm{E}-2$ & - & - & - \\
\hline 7 & 21 & 0.22 & $2.74 \mathrm{E}-3$ & $5.87 \mathrm{E}-2$ & $5.24 \mathrm{E}-3$ & 1.88 & 0.96 & 1.73 \\
\hline 8 & 43 & 3.75 & $6.07 \mathrm{E}-4$ & $2.75 \mathrm{E}-2$ & $1.17 \mathrm{E}-3$ & 2.02 & 1.02 & 2.00 \\
\hline 9 & 60 & 10.51 & $3.38 \mathrm{E}-4$ & $2.07 \mathrm{E}-2$ & $6.62 \mathrm{E}-4$ & 2.25 & 1.08 & 2.20 \\
\hline 10 & 73 & 23.52 & $2.22 \mathrm{E}-4$ & $1.68 \mathrm{E}-2$ & $4.37 \mathrm{E}-4$ & 1.90 & 0.94 & 1.87 \\
\hline 11 & 119 & 166.46 & $7.73 \mathrm{E}-5$ & $9.87 \mathrm{E}-3$ & $1.58 \mathrm{E}-4$ & 2.03 & 1.02 & 1.96 \\
\hline
\end{tabular}

Table 2: Isotropic test case. Key refers to the mesh defined in table 1; Iter is the number of solver iterations; CPU refers to the solver CPU time in seconds; err $_{\text {sol }}$, err $_{\text {grad }}$, err $_{\text {jump }}$ are the respective $L^{2}$-errors as defined above; $\alpha_{\text {sol }}, \alpha_{\text {grad }}, \alpha_{j u m p}$ are the orders of convergence of the solution, of the gradient and of the jump, respectively. 


\begin{tabular}{|c|c|c|c|c|c|c|c|c|}
\cline { 2 - 10 } \multicolumn{1}{c|}{} & \multicolumn{7}{|c|}{ Anisotropic Permeability: VAG } \\
\hline Key & Iter & CPU & err $_{\text {sol }}$ & err $_{\text {grad }}$ & err $_{\text {jump }}$ & $\alpha_{\text {sol }}$ & $\alpha_{\text {grad }}$ & $\alpha_{\text {jump }}$ \\
\hline Hexahedral Meshes (Cartesian) \\
\hline 1 & 7 & $6.32 \mathrm{E}-3$ & $8.78 \mathrm{E}-3$ & $1.98 \mathrm{E}-2$ & $8.69 \mathrm{E}-3$ & - & - & - \\
\hline 2 & 9 & $5.56 \mathrm{E}-2$ & $2.37 \mathrm{E}-3$ & $4.97 \mathrm{E}-3$ & $2.34 \mathrm{E}-3$ & 1.89 & 1.99 & 1.89 \\
\hline 3 & 14 & 0.67 & $6.15 \mathrm{E}-4$ & $1.24 \mathrm{E}-3$ & $6.06 \mathrm{E}-4$ & 1.95 & 2.00 & 1.95 \\
\hline 4 & 26 & 6.35 & $2.28 \mathrm{E}-4$ & $1.57 \mathrm{E}-4$ & $3.11 \mathrm{E}-4$ & 1.97 & 2.00 & 1.97 \\
\hline 5 & 47 & 62.65 & $3.95 \mathrm{E}-5$ & $7.78 \mathrm{E}-5$ & $3.89 \mathrm{E}-5$ & 1.99 & 2.00 & 1.99 \\
\hline Hexahedral & Meshes (perturbated & Cartesian $)$ & \\
\hline 1 & 7 & $4.92 \mathrm{E}-3$ & $1.40 \mathrm{E}-2$ & $5.45 \mathrm{E}-2$ & $1.39 \mathrm{E}-2$ & - & - & - \\
\hline 2 & 8 & $7.28 \mathrm{E}-2$ & $4.04 \mathrm{E}-3$ & $3.13 \mathrm{E}-2$ & $4.01 \mathrm{E}-3$ & 1.80 & 0.80 & 1.79 \\
\hline 3 & 14 & 0.89 & $1.18 \mathrm{E}-3$ & $1.50 \mathrm{E}-2$ & $1.17 \mathrm{E}-3$ & 1.77 & 1.05 & 1.77 \\
\hline 4 & 25 & 9.75 & $2.82 \mathrm{E}-4$ & $7.37 \mathrm{E}-3$ & $2.80 \mathrm{E}-4$ & 2.06 & 1.03 & 2.06 \\
\hline 5 & 45 & 98.75 & $7.20 \mathrm{E}-5$ & $3.64 \mathrm{E}-3$ & $7.14 \mathrm{E}-5$ & 1.97 & 1.01 & 1.97 \\
\hline Tetrahedral & Meshes & \multicolumn{7}{|c|}{} \\
\hline 6 & 7 & $1.95 \mathrm{E}-3$ & $2.73 \mathrm{E}-2$ & 0.13 & $2.70 \mathrm{E}-2$ & - & - & - \\
\hline 7 & 8 & $2.14 \mathrm{E}-2$ & $7.05 \mathrm{E}-3$ & $6.76 \mathrm{E}-2$ & $6.98 \mathrm{E}-3$ & 1.95 & 0.99 & 1.95 \\
\hline 8 & 15 & 0.38 & $2.56 \mathrm{E}-3$ & $3.92 \mathrm{E}-2$ & $2.53 \mathrm{E}-3$ & 1.35 & 0.73 & 1.36 \\
\hline 9 & 21 & 1.02 & $1.34 \mathrm{E}-3$ & $2.84 \mathrm{E}-2$ & $1.32 \mathrm{E}-3$ & 2.49 & 1.24 & 2.49 \\
\hline 10 & 25 & 2.24 & $9.26 \mathrm{E}-4$ & $2.22 \mathrm{E}-2$ & $9.14 \mathrm{E}-4$ & 1.66 & 1.10 & 1.67 \\
\hline 11 & 41 & 13.78 & $3.10 \mathrm{E}-4$ & $1.36 \mathrm{E}-2$ & $3.07 \mathrm{E}-4$ & 2.11 & 0.95 & 2.11 \\
\hline
\end{tabular}

\begin{tabular}{|c|c|c|c|c|c|c|c|c|}
\hline & \multicolumn{8}{|c|}{ Anisotropic Permeability: $H F V$} \\
\hline Key & Iter & CPU & $e r r_{s o l}$ & err $r_{\text {grad }}$ & $e r r_{j u m p}$ & $\alpha_{\text {sol }}$ & $\alpha_{\text {grad }}$ & $\alpha_{\text {jump }}$ \\
\hline \multicolumn{9}{|c|}{ Hexahedral Meshes (Cartesian) } \\
\hline 1 & 9 & $6.02 \mathrm{E}-3$ & $2.64 \mathrm{E}-2$ & $4.89 \mathrm{E}-2$ & $3.35 \mathrm{E}-2$ & - & - & - \\
\hline 2 & 16 & $8.48 \mathrm{E}-2$ & $7.02 \mathrm{E}-3$ & $1.43 \mathrm{E}-2$ & $8.30 \mathrm{E}-3$ & 1.91 & 1.78 & 2.01 \\
\hline 3 & 29 & 1.13 & $1.81 \mathrm{E}-3$ & $3.96 \mathrm{E}-3$ & $2.07 \mathrm{E}-3$ & 1.95 & 1.85 & 2.00 \\
\hline 4 & 55 & 16.55 & $4.60 \mathrm{E}-4$ & $1.07 \mathrm{E}-3$ & $5.19 \mathrm{E}-4$ & 1.98 & 1.89 & 2.00 \\
\hline 5 & 108 & 248.20 & $1.16 \mathrm{E}-4$ & $2.86 \mathrm{E}-4$ & $1.30 \mathrm{E}-4$ & 1.99 & 1.91 & 2.00 \\
\hline \multicolumn{9}{|c|}{ Hexahedral Meshes (perturbated Cartesian) } \\
\hline 1 & 8 & $1.20 \mathrm{E}-2$ & $2.99 \mathrm{E}-2$ & $8.77 \mathrm{E}-2$ & $3.94 \mathrm{E}-2$ & - & - & - \\
\hline 2 & 15 & 0.15 & $6.92 \mathrm{E}-3$ & $4.22 \mathrm{E}-2$ & $8.30 \mathrm{E}-3$ & 2.11 & 1.05 & 2.24 \\
\hline 3 & 28 & 1.87 & $1.56 \mathrm{E}-3$ & $1.96 \mathrm{E}-2$ & $1.74 \mathrm{E}-3$ & 2.14 & 1.10 & 2.24 \\
\hline 4 & 55 & 23.34 & $4.20 \mathrm{E}-4$ & $9.57 \mathrm{E}-3$ & $4.58 \mathrm{E}-4$ & 1.89 & 1.03 & 1.92 \\
\hline 5 & 102 & 371.45 & $1.12 \mathrm{E}-4$ & $5.13 \mathrm{E}-3$ & $1.20 \mathrm{E}-4$ & 1.89 & 0.89 & 1.93 \\
\hline \multicolumn{9}{|c|}{ Tetrahedral Meshes } \\
\hline 6 & 10 & $1.41 \mathrm{E}-2$ & $1.77 \mathrm{E}-2$ & 0.14 & $1.79 \mathrm{E}-2$ & - & - & - \\
\hline 7 & 19 & 0.26 & $4.86 \mathrm{E}-3$ & $7.13 \mathrm{E}-2$ & $4.75 \mathrm{E}-3$ & 1.86 & 0.98 & 1.91 \\
\hline 8 & 37 & 4.56 & $1.28 \mathrm{E}-3$ & $3.63 \mathrm{E}-2$ & $1.21 \mathrm{E}-3$ & 1.79 & 0.90 & 1.83 \\
\hline 9 & 47 & 12.16 & $6.92 \mathrm{E}-4$ & $2.62 \mathrm{E}-2$ & $6.66 \mathrm{E}-4$ & 2.35 & 1.25 & 2.28 \\
\hline 10 & 63 & 27.96 & $4.75 \mathrm{E}-4$ & $2.16 \mathrm{E}-2$ & $4.68 \mathrm{E}-4$ & 1.69 & 0.88 & 1.59 \\
\hline 11 & 105 & 189.66 & $1.65 \mathrm{E}-4$ & $1.28 \mathrm{E}-2$ & $1.58 \mathrm{E}-4$ & 2.04 & 1.00 & 2.09 \\
\hline
\end{tabular}

Table 3: Anisotropic test case. Key refers to the mesh defined in table 1; Iter is the number of solver iterations; CPU refers to the solver CPU time in seconds; $e r r_{\text {sol }}, e r r_{\text {grad }}, e r r_{j u m p}$ are the respective $L^{2}$-errors as defined above; $\alpha_{\text {sol }}, \alpha_{\text {grad }}, \alpha_{j u m p}$ are the orders of convergence w.r.t. $\# \mathcal{M}^{-\frac{1}{3}}$ of the solution, of the gradient and of the jump, respectively. 
The test case shows that, on Cartesian grids, we obtain, as expected, convergence of order 2 for both, the solution and it's gradient. For tetrahedral grids, we obtain convergence of order 2 for the solution and convergence of order 1 for it's gradient. We observe that the VAG scheme is more efficient than the HFV scheme and this observation gets more obvious with increasing anisotropy. Comparing the precision of the discrete solution (and it's gradient) for VAG and HFV on a given mesh, we see that on hexahedral meshes, the advantage is on the side of VAG, whereas on tetrahedral meshes HFV is more precise (but much more expensive). On a given mesh, HFV is usually (see [7]) more accurate than VAG both for tetrahedral and Cartesian meshes. This is not true in the test cases using Cartesian meshes maybe due to the higher number for VAG than for $\mathrm{HFV}$ of d.o.f. at the interfaces $\Gamma_{\alpha}$ on the matrix side. For perturbated Cartesian grids, we obtain convergence of order 2 for the solution and convergence of order 1 for it's gradient for the VAG scheme, which is able to deal with non-planar faces. However, the HFV scheme reveals a slight deterioration of the convergence rate, for the solution and in particular for it's gradient, which is not surprising, since HFV is not consistent on this family of meshes.

It is also worth mentioning that we have conducted this test for VAG with lumped $m f$-fluxes, and observed that that there is literally no difference compared to VAG-FE in the ordinary version, concerning accuracy and convergence rate.

\section{Conclusion}

In this work, we extended the framework of gradient schemes (see [14]) to the model problem (2.1) of stationary Darcy flow through fractured porous media and gave numerical analysis results for this general framework.

The model problem (an extension to a network of fractures of a PDE model presented in [18], [21] and [4]) takes heterogeneities and anisotropy of the porous medium into account and involves a complex network of planar fractures, which might act either as barriers or as drains.

We also extended the VAG and HFV schemes to our model, where fractures acting as barriers force us to allow for pressure jumps across the fracture network. We developed two versions of VAG schemes, the conforming finite element version and the non-conforming control volume version, the latter particularly adapted for the treatment of material interfaces (cf. [16]). We showed, furthermore, that both versions of VAG schemes, as well as the proposed non-conforming HFV schemes, are incorporated by the gradient scheme's framework. Then, we applied the results for gradient schemes on VAG and HFV to obtain convergence, and, in particular, convergence of order 1 for "piecewise regular" solutions.

For implementation purposes and in view of the application to multi-phase flow, we also propose a uniform Finite Volume formulation for VAG and HFV schemes in the appendix. The numerical experiments on a family of analytical solutions show that the VAG scheme offers a better compromise between accuracy and CPU time than the HFV scheme especially for anisotropic problems.

Acknowledgements: the authors would like to thank TOTAL for its financial support and for allowing the publication of this work. We also thank J. Droniou from Monash University for fruitful discussions during the elaboration of this paper. 


\section{References}

[1] Adams, R. A., 1975, Sobolev Spaces, Academic Press New York San Francisco London.

[2] Ahmed, R., Edwards, M.G., Lamine, S., Huisman, B.A.H., 2015 Control-volume distributed multi-point flux approximation coupled with a lower-dimensional fracture model, J. Comp. Physics, 284, 462-489.

[3] Alboin, C., Jaffré, J., Roberts, J., Serres, C., 2002 Modeling fractures as interfaces for flow and transport in porous media, Fluid flow and transport in porous media, 295, 13-24.

[4] Angot, P., Boyer, F., Hubert, F., 2009 Asymptotic and numerical modelling of flows in fractured porous media, ESAIM Mathematical Modelling and Numerical Analysis 23, 239275 .

[5] Antonietti, P.F., Formaggia, L., Scotti, A., Verani, M., Verzotti, N., 2015, Mimetic Finite Difference Approximation of flows in Fractured Porous Media, MOX Report No 20/2015.

[6] Brenner, K., Groza, M., Guichard, C., Masson, R., 2015, Vertex Approximate Gradient Scheme for Hybrid Dimensional Two-Phase Darcy Flows in Fractured Porous Media, ESAIM Mathematical Modelling and Numerical Analysis, 49, 303-330.

[7] Brenner, K., Groza, M., Guichard, C., Lebeau, G. and Masson, R., 2015, Gradient discretization of Hybrid Dimensional Darcy Flows in Fractured Porous Media, Numerische Mathematik.

[8] Brenner, K., Masson, R., 2013, Convergence of a Vertex centered Discretization of TwoPhase Darcy flows on General Meshes, Int. Journal of Finite Volume Methods.

[9] Brezzi F., Lipnikov K., Simoncini V., 2005, A family of mimetic finite difference methods on polygonal and polyhedral meshes, Mathematical Models and Methods in Applied Sciences, 15 (10), 1533-1552.

[10] D'Angelo, C., Scotti, A., 2012, A mixed finite element method for Darcy flow in fractured porous media with non-matching grids, ESAIM Mathematical Modelling and Numerical Analysis 46 (2) 465-489.

[11] Droniou, J., Eymard, R., Gallouët, T., Guichard, C., Herbin, R., Gradient schemes for elliptic and parabolic problems, Springer, in preparation.

[12] Droniou, J., Eymard, R., Gallouët, T., Herbin, R., 2013, Gradient schemes: a generic framework for the discretisation of linear, nonlinear and nonlocal elliptic and parabolic equations. Math. Models Methods Appl. Sci. 23 (13) 2395-2432.

[13] Droniou, J., Eymard, R., Gallouët, T., Herbin, R., 2010, A Unified Approach to Mimetic Finite Difference, Hybrid Finite Volume and Mixed Finite Volume Methods, Math. Models and Methods in Appl. Sci. 20 (2) 265-295.

[14] Eymard, R., Guichard, C. and Herbin, R., 2010, Small-stencil 3D schemes for diffusive flows in porous media, ESAIM: Mathematical Modelling and Numerical Analysis, 46, 265290. 
[15] Eymard, R., Gallouët, T., Herbin, R., 2010, Discretization of heterogeneous and anisotropic diffusion problems on general nonconforming meshes SUSHI: a scheme using stabilisation and hybrid interfaces, IMA J Numer Anal 30 (4), 1009-1043.

[16] Eymard, R., Guichard, C., Herbin, R., Masson, R., 2012, Vertex centered discretization of compositional multiphase darcy flows on general meshes, Comp. Geosciences, 16, 987-1005.

[17] Faille, I., Fumagalli, A., Jaffré, J., Robert, J., 2015, Reduced models for flow in porous media containing faults with discretization using hybrid finite volume schemes, https://halifp.archives-ouvertes.fr/hal-01162048

[18] Flauraud, E., Nataf, F., Faille, I., Masson, R., 2003, Domain Decomposition for an asymptotic geological fault modeling, Comptes Rendus à l'académie des Sciences, Mécanique, $331,849-855$.

[19] Formaggia, L., Fumagalli, A., Scotti, A., Ruffo, P., 2014, A reduced model for Darcy's problem in networks of fractures, ESAIM Mathematical Modelling and Numerical Analysis 48 (4), 1089-1116.

[20] Karimi-Fard, M., Durlovski, L.J., Aziz, K., 2004, An efficient discrete-fracture model applicable for general-purpose reservoir simulators, SPE journal.

[21] Martin, V., Jaffré, J., Roberts, J. E., 2005, Modeling fractures and barriers as interfaces for flow in porous media, SIAM J. Sci. Comput. 26 (5), 1667-1691.

[22] Raviart, P.A., 1992, Résolution Des Modèles Aux Dérivées Partielles, Ecole Polytechnique, Département de Mathématiques appliquées.

[23] Reichenberger, V., Jakobs, H., Bastian, P., Helmig, R., 2006, A mixed-dimensional finite volume method for multiphase flow in fractured porous media. Adv. Water Resources 29 (7) 1020-1036.

[24] Saad, Y., 2003, Iterative Methods for Sparse Linear Systems, 2nd edition, SIAM, Philadelphia, PA.

[25] Saad, Y., 2010, ITSOL Library, http://www-users.cs.umn.edu/ saad/software/

[26] Sandve, T.H., Berre, I., Nordbotten, J.M., 2012, An efficient multi-point flux approximation method for Discrete Fracture-Matrix simulations, JCP 231, 3784-3800.

[27] Tartar, L., 2007, An Introduction to Sobolev Spaces and Interpolation Spaces, SpringerVerlag Berlin Heidelberg.

[28] Tunc, X., Faille, I., Gallouët, T., Cacas, M.C., Havé, P., 2012, A model for conductive faults with non matching grids, Comp. Geosciences, 16, 277-296. 


\section{A Density Results for $V^{0}$ and $W$}

Let us first state the following Lemma that will be used to prove the density of $C_{W_{m}}^{\infty} \times C_{W_{f}}^{\infty}$ in $W$.

Lemma A.1 Let $v_{m} \in L^{2}(\Omega), v_{f} \in L^{2}(\Gamma), G \in L^{2}(\Omega)^{d}, H \in L^{2}(\Gamma)^{d-1}$ and $J_{\alpha} \in L^{2}\left(\Gamma_{\alpha}\right), \alpha \in$ $\chi$ such that

$$
\int_{\Omega}\left(G \cdot \mathbf{q}_{m}+v_{m} \operatorname{div} \mathbf{q}_{m}\right) \mathrm{d} \mathbf{x}+\int_{\Gamma}\left(H \cdot \mathbf{q}_{f}+v_{f} \operatorname{div}_{\tau} \mathbf{q}_{f}\right) \mathrm{d} \tau(\mathbf{x})+\sum_{\alpha \in \chi} \int_{\Gamma_{\alpha}} \gamma_{\mathbf{n}, \alpha} \mathbf{q}_{m} \mathrm{~d} \tau(\mathbf{x})\left(J_{\alpha}-v_{f}\right)=0
$$

for all $\left(\mathbf{q}_{m}, \mathbf{q}_{f}\right) \in C_{W_{m}}^{\infty} \times C_{W_{f}}^{\infty}$. Then holds $\left(v_{m}, v_{f}\right) \in V^{0},(G, H)=\left(\nabla v_{m}, \nabla_{\tau} v_{f}\right)$ and $J_{\alpha}=$ $v_{f}-\gamma_{\alpha} v_{m}$ for $\alpha \in \chi$.

Proof Firstly, for all $\mathbf{q}_{m} \in C_{0}^{\infty}(\Omega \backslash \bar{\Gamma})^{d}$, we have

$$
\int_{\Omega}\left(G \cdot \mathbf{q}_{m}+v_{m} \operatorname{div} \mathbf{q}_{m}\right) \mathrm{d} \mathbf{x}=0
$$

and therefore $v_{m} \in H^{1}(\Omega \backslash \bar{\Gamma})$ and $\nabla v_{m}=G$.

For a.e. $\mathbf{x} \in \partial \Omega$, there exists an open planar domain $\omega \subset \subset \partial \Omega \backslash \partial \Gamma$ containing $\mathbf{x}$ such that for all $f \in C_{0}^{\infty}(\omega)$ there exists $\mathbf{q}_{m} \in C_{W_{m}}^{\infty}$ with

$$
\begin{aligned}
& \gamma_{\mathbf{n}_{\partial \Omega}} \mathbf{q}_{m}= \begin{cases}f & \text { on } \omega, \\
0 & \text { on } \partial \Omega \backslash \omega,\end{cases} \\
& \gamma_{\mathbf{n}, \alpha} \mathbf{q}_{m}=0 \quad \text { on } \Gamma_{\alpha}, \alpha \in \chi,
\end{aligned}
$$

where $\gamma_{\mathbf{n}_{\partial \Omega}}$ denotes the normal trace operator on the boundary of $\Omega$. From (A.1), taking $\mathbf{q}_{f}=0$, we obtain

$$
0=\int_{\Omega}\left(\nabla v_{m} \cdot \mathbf{q}_{m}+v_{m} \operatorname{div} \mathbf{q}_{m}\right) \mathrm{d} \mathbf{x}=\int_{\partial \Omega} \gamma_{\partial \Omega} v_{m} \gamma_{\mathbf{n}_{\partial \Omega}} \mathbf{q}_{m} \mathrm{~d} \tau(\mathbf{x})=\int_{\omega} \gamma_{\partial \Omega} v_{m} f \mathrm{~d} \tau(\mathbf{x}) .
$$

where $\gamma_{\partial \Omega}$ denotes the trace operator on the boundary of $\Omega$. We deduce $\gamma_{\partial \Omega} v_{m}=0$ a.e. on $\partial \Omega \backslash \partial \Gamma$. Hence $v_{m} \in H_{\partial \Omega}^{1}(\Omega \backslash \bar{\Gamma})$.

Further, for a.e. $\mathbf{x} \in \Gamma_{\alpha}$ there exists an open planar domain $\omega_{\alpha} \subset \subset \Gamma_{\alpha}$ containing $\mathbf{x}$ such that for all $g \in C_{0}^{\infty}\left(\omega_{\alpha}\right)$ there exists $\mathbf{q}_{m} \in C_{W_{m}}^{\infty}$ with

$$
\begin{aligned}
& \gamma_{\mathbf{n}, \alpha} \mathbf{q}_{m}= \begin{cases}g & \text { on } \omega_{\alpha}, \\
0 & \text { on } \Gamma_{\alpha} \backslash \omega_{\alpha},\end{cases} \\
& \gamma_{\mathbf{n}, \beta} \mathbf{q}_{m}=0 \quad \text { on } \Gamma_{\beta} \text {, for } \beta \neq \alpha \text {, } \\
& \gamma_{\mathbf{n}_{\partial \Omega}} \mathbf{q}_{m}=0 \text { on } \partial \Omega \text {. }
\end{aligned}
$$

From (A.1) we obtain

$$
\begin{aligned}
0 & =\int_{\Omega}\left(\nabla v_{m} \cdot \mathbf{q}_{m}+v_{m} \operatorname{div} \mathbf{q}_{m}\right) \mathrm{d} \mathbf{x}+\sum_{\alpha \in \chi} \int_{\Gamma_{\alpha}} \gamma_{\mathbf{n}, \alpha} \mathbf{q}_{m}\left(J_{\alpha}-v_{f}\right) \mathrm{d} \tau(\mathbf{x}) \\
& =\int_{\Gamma_{\alpha}} \gamma_{\mathbf{n}, \alpha} \mathbf{q}_{m}\left(J_{\alpha}-v_{f}+\gamma_{\alpha} v_{m}\right) \mathrm{d} \tau(\mathbf{x})=\int_{\omega_{\alpha}} g\left(J_{\alpha}-v_{f}+\gamma_{\alpha} v_{m}\right) \mathrm{d} \tau(\mathbf{x})
\end{aligned}
$$


We deduce $J_{\alpha}=v_{f}-\gamma_{\alpha} v_{m}$ a.e. on $\Gamma_{\alpha}, \alpha \in \chi$.

Next, for all $\mathbf{q}_{f} \in C_{0}^{\infty}\left(\Gamma_{i}\right)^{d-1}, i \in I$, we have from (A.1)

$$
\int_{\Gamma_{i}}\left(H \cdot \mathbf{q}_{f}+v_{f} \operatorname{div} \mathbf{q}_{f}\right) \mathrm{d} \tau(\mathbf{x})=0
$$

and therefore $v_{f} \uparrow_{\Gamma_{i}} \in H^{1}\left(\Gamma_{i}\right)$ for $i \in I$ and $\nabla_{\tau_{i}} v_{f} \uparrow_{\Gamma_{i}}=H \uparrow_{\Gamma_{i}}$.

Let $i, j \in I, i \neq j$. For a.e. $\mathbf{x} \in \Sigma_{i, j} \backslash \Sigma_{i, 0}$ there exists an open interval $c_{i j} \subset \subset \Sigma_{i, j} \backslash \Sigma_{i, 0}$ containing $\mathbf{x}$ such that for all $h \in C_{0}^{\infty}\left(c_{i j}\right)$ there exists $s \in C_{W_{f}}^{\infty}$ with

$$
\begin{gathered}
\gamma_{\mathbf{n}_{\Sigma_{i}}} s=h=-\gamma_{\mathbf{n}_{\Sigma_{j}}} s \quad \text { on } c_{i j}, \\
\gamma_{\mathbf{n}_{\Sigma_{k}}} s=0 \quad \text { on } \Sigma_{k} \backslash c_{i j}, k \in I .
\end{gathered}
$$

From (A.1) we obtain

$$
0=\int_{\Gamma}\left(\nabla_{\tau} v_{f} \cdot s+v_{f} \operatorname{div}_{\tau} s\right) \mathrm{d} \tau(\mathbf{x})=\int_{c_{i j}}\left(\gamma_{\Sigma_{i}} v_{f}-\gamma_{\Sigma_{j}} v_{f}\right) \gamma_{\mathbf{n}_{\Sigma_{i}}} s \mathrm{~d} \sigma(\mathbf{x}),
$$

$\mathrm{d} \sigma(\mathbf{x})$ denoting the $d-2$ dimensional Lebesgue measure on $\Sigma$. We deduce $\gamma_{\Sigma_{i}} v_{f}=\gamma_{\Sigma_{j}} v_{f}$ a.e. on $\Sigma_{i, j} \backslash \Sigma_{i, 0}, i, j \in I, i \neq j$. The proof of $\gamma_{\Sigma_{0}} v_{f}=0$ a.e. on $\Sigma_{0}$ goes analogously. Hence $v_{f} \in H_{\Sigma_{0}}^{1}(\Gamma)$.

Proof of Proposition 2.2 Firstly from Proposition 2.1, note that on $V^{0}$, the norm $\|\cdot\|_{V^{0}}$ is equivalent to the standart norm $\|\cdot\|_{H^{1}(\Omega)}+\|\cdot\|_{H^{1}(\Gamma)}$. The density of $C_{\Omega}^{\infty}$ in $H_{\partial \Omega}^{1}(\Omega \backslash \bar{\Gamma})$ being a classical result, we are concerned to prove the density of $C_{\Gamma}^{\infty}$ in $H_{\Sigma_{0}}^{1}(\Gamma)$ in the following. Since $H_{\Sigma_{0}}^{1}(\Gamma) \subset \gamma_{\Gamma}\left(H_{0}^{1}(\Omega)\right)$, we can define $\tilde{V}^{0}=\gamma_{\Gamma}^{-1}\left(H_{\Sigma_{0}}^{1}(\Gamma)\right) \subset H_{0}^{1}(\Omega)$. In Proposition 2 of [7] it is shown that $C_{0}^{\infty}(\Omega)$ is dense in $\left(\tilde{V}^{0},\|\cdot\|_{H^{1}(\Omega)}+\left\|\gamma_{\Gamma} \cdot\right\|_{H^{1}(\Gamma)}\right)$. Hence $C_{\Gamma}^{\infty}$ is dense in $\left(H_{\Sigma_{0}}^{1}(\Gamma),\|\cdot\|_{H^{1}(\Gamma)}\right)$.

Proof of Proposition 2.3 Since $W_{f}$ is a closed subspace of the Hilbert space $\prod_{i \in I} H_{\text {div }}\left(\Gamma_{i}\right)$, any linear form $l \in W_{f}^{\prime}$ is the restriction to $W_{f}$ of a linear form still denoted by $l$ in $\prod_{i \in I} H_{\text {div }}\left(\Gamma_{i}\right)^{\prime}$. Then, for some $f \in L^{2}(\Gamma)$ and $\boldsymbol{g} \in L^{2}(\Gamma)^{d-1}$ holds

$$
<l, \mathbf{q}_{f}>=\sum_{i \in I} \int_{\Gamma_{i}}\left(\boldsymbol{g} \cdot \mathbf{q}_{f}+f \cdot \operatorname{div}_{\tau} \mathbf{q}_{f}\right) \mathrm{d} \tau(\mathbf{x}),
$$

for all $\mathbf{q}_{f} \in W_{f}$. Let us assume now that $\langle l, \varphi\rangle=0$ for all $\varphi \in C_{W_{f}}^{\infty}$. Corresponding to Lemma A.1 holds $f \in H_{\Sigma_{0}}^{1}(\Gamma)$ and $\boldsymbol{g}=\nabla_{\tau} f$. From the definition of $W_{f}$ we conclude that $<l, \mathbf{q}_{f}>=0$ for all $\mathbf{q}_{f} \in W_{f}$.

Let now $l \in W_{m}^{\prime}$. Then there exist $f \in L^{2}(\Omega), \boldsymbol{g} \in L^{2}(\Omega)^{d}$ and $h_{\alpha} \in L^{2}\left(\Gamma_{\alpha}\right)(\alpha \in \chi)$, such that

$$
<l, \mathbf{q}_{m}>=\int_{\Omega}\left(\boldsymbol{g} \cdot \mathbf{q}_{m}+f \cdot \operatorname{div} \mathbf{q}_{m}\right) \mathrm{d} \mathbf{x}+\sum_{\alpha \in \chi} \int_{\Gamma_{\alpha}} h_{\alpha} \gamma_{\mathbf{n}, \alpha} \mathbf{q}_{m} \mathrm{~d} \tau(\mathbf{x}),
$$

for all $\mathbf{q}_{m} \in W_{m}$. Furthermore, let us assume that $\langle l, \boldsymbol{\varphi}\rangle=0$ for all $\boldsymbol{\varphi} \in C_{W_{m}}^{\infty}$. From Lemma A.1 we deduce that $f \in H_{\partial \Omega}^{1}(\Omega \backslash \bar{\Gamma})$, that $\boldsymbol{g}=\nabla f$ and that $h_{\alpha}=\gamma_{\alpha} f(\alpha \in \chi)$. Using this, we conclude, again by the rule of partial integration, that $\left\langle l, \mathbf{q}_{m}\right\rangle=0$ for all $\mathbf{q}_{m} \in W_{m}$. 


\section{B Finite Volume Formulation for $V A G$ and $H F V$ Schemes}

The VAG and HFV schemes can be written in a flux conservative formulation, both with matrix-matrix fluxes local to each cell, fracture-fracture fluxes local to each fracture face and two point matrix-fracture fluxes (for HFV and VAG-CV). These fluxes are particularly useful for the discetization of the monophasic model coupled with a transport equation. Such systems typicaly arise in models for diphasic flow through porous media. In this section, we present a unified Finite Volume Formulation for both schemes.

For $K \in \mathcal{M}$ let

$$
\operatorname{dof}_{K}=\left\{\begin{array}{l}
\left\{\bar{K}_{s}, s \in \mathcal{V}_{K}\right\} \cup\left\{K_{\sigma}, \sigma \in \mathcal{F}_{K} \cap \mathcal{F}_{\Gamma}\right\} \text { for } V A G \\
\left\{\bar{K}_{\sigma}, \sigma \in \mathcal{F}_{K}\right\} \text { for } H F V .
\end{array}\right.
$$

Analogously, in the fracture domain, for $\sigma \in \mathcal{F}_{\Gamma}$ let

$$
d o f_{\sigma}=\left\{\begin{array}{l}
\mathcal{V}_{\sigma} \text { for } V A G \\
\mathcal{E}_{\sigma} \text { for } H F V
\end{array}\right.
$$

Then, for any $\nu \in d o f_{K}$ the discrete matrix-matrix-fluxes are defined as

$$
F_{K \nu}\left(u_{\mathcal{D}_{m}}\right)=\sum_{\nu^{\prime} \in \operatorname{dof} f_{K}}\left(\int_{K} \Lambda_{m} \nabla_{\mathcal{D}_{m}} \mathfrak{e}_{\nu} \nabla_{\mathcal{D}_{m}} \mathfrak{e}_{\nu^{\prime}} \mathrm{d} \mathbf{x}\right)\left(u_{K}-u_{\nu^{\prime}}\right)
$$

such that $\int_{\Omega} \Lambda_{m} \nabla_{\mathcal{D}_{m}} u_{\mathcal{D}_{m}} \nabla_{\mathcal{D}_{m}} v_{\mathcal{D}_{m}} \mathrm{~d} \mathbf{x}=\sum_{K \in \mathcal{M}} \sum_{\nu \in d o f_{K}} F_{K \nu}\left(u_{\mathcal{D}_{m}}\right)\left(v_{K}-v_{\nu}\right)$. For all $\nu \in d o f_{\sigma}$ the discrete fracture-fracture-fluxes are defined as

$$
F_{\sigma \nu}\left(u_{\mathcal{D}_{f}}\right)=\sum_{\nu^{\prime} \in \text { dof } f_{\sigma}}\left(\int_{\sigma} \Lambda_{f} \nabla_{\mathcal{D}_{f}} \mathfrak{e}_{\nu} \nabla_{\mathcal{D}_{f}} \mathfrak{e}_{\nu^{\prime}} \mathrm{d} \tau_{f}(\mathbf{x})\right)\left(u_{\sigma}-u_{\nu^{\prime}}\right),
$$

such that $\int_{\Gamma} \Lambda_{f} \nabla_{\mathcal{D}_{f}} u_{\mathcal{D}_{f}} \nabla_{\mathcal{D}_{f}} v_{\mathcal{D}_{f}} \mathrm{~d} \tau_{f}(\mathbf{x})=\sum_{\sigma \in \mathcal{F}_{\Gamma}} \sum_{\nu \in \text { dof }} F_{\sigma \nu}\left(u_{\mathcal{D}_{f}}\right)\left(v_{\sigma}-v_{\nu}\right)$. To take interactions of the matrix and the fracture domain into account we introduce the set of matrix-fracture $(\mathrm{mf})$ connectivities

$$
\mathcal{C}=\left\{\left(\nu_{m}, \nu_{f}\right) \mid \nu_{m} \in d o f_{\mathcal{D}_{m}}^{\Gamma}, \nu_{f} \in d o f_{\mathcal{D}_{f}} \text { s.t. } \mathbf{x}_{\nu_{m}}=\mathbf{x}_{\nu_{f}}\right\}
$$

with $d o f_{\mathcal{D}_{m}}^{\Gamma}=\left\{\nu \in \operatorname{dof}_{\mathcal{D}_{m}} \mid \mathbf{x}_{\nu} \in \bar{\Gamma}\right\}$. The $m f$-fluxes are built such that

$$
\begin{aligned}
& a_{\mathcal{D}_{m f}}\left(\left(u_{\mathcal{D}_{m}}, u_{\mathcal{D}_{f}}\right),\left(v_{\mathcal{D}_{m}}, v_{\mathcal{D}_{f}}\right)\right)=\sum_{\left(\nu_{m}, \nu_{f}\right) \in \mathcal{C}} F_{\nu_{m} \nu_{f}}\left(u_{\mathcal{D}_{m}}, u_{\mathcal{D}_{f}}\right)\left(v_{\nu_{m}}-v_{\nu_{f}}\right) \\
& =\sum_{i \in I} \int_{\Gamma_{i}} \frac{T_{f}}{2 \xi-1} \sum_{(\alpha, \beta) \in\left\{\left(\alpha^{ \pm}(i), \alpha \mp(i)\right)\right\}}\left(\xi \llbracket u_{\mathcal{D}} \rrbracket_{\mathcal{D}}^{\alpha}+(1-\xi) \llbracket u_{\mathcal{D}} \rrbracket_{\mathcal{D}}^{\beta}\right) \llbracket v_{\mathcal{D}} \rrbracket_{\mathcal{D}}^{\alpha} \mathrm{d} \tau(\mathbf{x}),
\end{aligned}
$$

for all $\left(v_{\mathcal{D}_{m}}, v_{\mathcal{D}_{f}}\right) \in X_{\mathcal{D}}$. For all $\sigma \in \mathcal{F}_{\Gamma}$ and $K \in \mathcal{M}_{\sigma}$, let us denote by $\alpha(K, \sigma)$ the unique $\alpha \in \chi$ such that $\sigma \in \mathcal{F}_{\alpha}$ and $\mathbf{n}_{\alpha}=\mathbf{n}_{K, \sigma}$. Let us also set for all $\sigma \in \mathcal{F}_{\Gamma},(\chi \times \chi)_{\sigma}=\{(\alpha(K, \sigma), \alpha(L, \sigma))$, $(\alpha(L, \sigma), \alpha(K, \sigma))\}$ with $\mathcal{M}_{\sigma}=\{K, L\}$. Then, holds

$a_{\mathcal{D}_{m f}}\left(\left(u_{\mathcal{D}_{m}}, u_{\mathcal{D}_{f}}\right),\left(v_{\mathcal{D}_{m}}, v_{\mathcal{D}_{f}}\right)\right)=\sum_{\sigma \in \mathcal{F}_{\Gamma}} \sum_{(\alpha, \beta) \in(\chi \times \chi)_{\sigma}} \int_{\sigma} \frac{T_{f}}{2 \xi-1}\left(\xi \llbracket u_{\mathcal{D}} \rrbracket_{\mathcal{D}}^{\alpha}+(1-\xi) \llbracket u_{\mathcal{D}} \rrbracket_{\mathcal{D}}^{\beta}\right) \llbracket v_{\mathcal{D}} \rrbracket_{\mathcal{D}}^{\alpha} \mathrm{d} \tau(\mathbf{x})$.

For all $\sigma \in \mathcal{F}_{\Gamma}, K \in \mathcal{M}_{\sigma}$ and $\mathbf{x} \in \sigma$, let us notice that, for the VAG scheme, one has $\llbracket \mathfrak{e}_{K_{\sigma}}+\mathfrak{e}_{\sigma} \rrbracket_{\mathcal{D}}^{\alpha(K, \sigma)}(\mathbf{x})=0$, and $\llbracket \mathfrak{e}_{\bar{K}_{s}}+\mathfrak{e}_{s} \rrbracket_{\mathcal{D}}^{\alpha(K, \sigma)}(\mathbf{x})=0$ for all $s \in \mathcal{V}_{\sigma}$, and for the HFV scheme, 
one has $\llbracket \mathfrak{e}_{\bar{K}_{\sigma}}+\mathfrak{e}_{\sigma} \rrbracket_{\mathcal{D}}^{\alpha(K, \sigma)}(\mathbf{x})=0$. It result after some computations that the VAG matrix fracture fluxes are defined by

$$
\begin{aligned}
F_{K_{\sigma} \sigma}\left(u_{\mathcal{D}_{m}}, u_{\mathcal{D}_{f}}\right)= & \sum_{s \in \mathcal{V}_{\sigma}}\left(\int_{\sigma} \frac{T_{f}}{2 \xi-1}\left(\llbracket \mathfrak{e}_{\sigma} \rrbracket_{\mathcal{D}}^{\alpha(K, \sigma)}\right)\left(\llbracket \mathfrak{e}_{s} \rrbracket_{\mathcal{D}}^{\alpha(K, \sigma)}\right) \mathrm{d} \tau(\mathbf{x})\right)\left(\xi u_{\bar{K}_{s}}+(1-\xi) u_{\bar{L}_{s}}-u_{s}\right) \\
& +\left(\int_{\sigma} \frac{T_{f}}{2 \xi-1}\left(\llbracket \mathfrak{e}_{\sigma} \rrbracket_{\mathcal{D}}^{\alpha(K, \sigma)}\right)^{2} \mathrm{~d} \tau(\mathbf{x})\right)\left(\xi u_{K_{\sigma}}+(1-\xi) u_{L_{\sigma}}-u_{\sigma}\right),
\end{aligned}
$$

for all $\sigma \in \mathcal{F}_{\Gamma}, \mathcal{M}_{\sigma}=\{K, L\}$, and by

$$
\begin{aligned}
F_{\bar{Q}_{s} s}\left(u_{\mathcal{D}_{m}}, u_{\mathcal{D}_{f}}\right)= & \sum_{\sigma \in\left(\cup_{Q \in \bar{Q}_{s}} \mathcal{F}_{Q}\right) \cap \mathcal{F}_{s} \cap \mathcal{F}_{\Gamma}} \sum_{K \in \mathcal{M}_{\sigma} \cap \bar{Q}_{s}, L \in \mathcal{M}_{\sigma} \backslash\{K\}}\{ \\
& \left(\int_{\sigma} \frac{T_{f}}{2 \xi-1}\left(\llbracket \mathfrak{e}_{s} \rrbracket_{\mathcal{D}}^{\alpha(K, \sigma)}\right)^{2} \mathrm{~d} \tau(\mathbf{x})\right)\left(\xi u_{\bar{K}_{s}}+(1-\xi) u_{\bar{L}_{s}}-u_{s}\right) \\
& +\sum_{s^{\prime} \in \mathcal{V}_{\sigma} \mid s s^{\prime} \in \mathcal{E}_{\sigma}}\left(\int_{\sigma} \frac{T_{f}}{2 \xi-1}\left(\llbracket \mathfrak{e}_{s^{\prime}} \rrbracket_{\mathcal{D}}^{\alpha(K, \sigma)}\right)\left(\llbracket \mathfrak{e}_{s} \rrbracket_{\mathcal{D}}^{\alpha(K, \sigma)}\right) \mathrm{d} \tau(\mathbf{x})\right)\left(\xi u_{\bar{K}_{s^{\prime}}}+(1-\xi) u_{\bar{L}_{s^{\prime}}}-u_{s^{\prime}}\right) \\
& \left.+\left(\int_{\sigma} \frac{T_{f}}{2 \xi-1}\left(\llbracket \mathfrak{e}_{\sigma} \rrbracket_{\mathcal{D}}^{\alpha(K, \sigma)}\right)\left(\llbracket \mathfrak{e}_{s} \rrbracket_{\mathcal{D}}^{\alpha(K, \sigma)}\right) \mathrm{d} \tau(\mathbf{x})\right)\left(\xi u_{K_{\sigma}}+(1-\xi) u_{L_{\sigma}}-u_{\sigma}\right)\right\},
\end{aligned}
$$

for all $s \in \mathcal{V}_{\Gamma}, \bar{Q}_{s} \in \overline{\mathcal{M}}_{s}$. Similarly the HFV matrix fracture fluxes are defined by

$$
F_{\bar{K}_{\sigma} \sigma}\left(u_{\mathcal{D}_{m}}, u_{\mathcal{D}_{f}}\right)=\frac{1}{2 \xi-1}\left(\int_{\sigma} T_{f}(\mathbf{x}) \mathrm{d} \tau(\mathbf{x})\right)\left(\xi u_{K_{\sigma}}+(1-\xi) u_{L_{\sigma}}-u_{\sigma}\right),
$$

for all $\sigma \in \mathcal{F}_{\Gamma}, \mathcal{M}_{\sigma}=\{K, L\}$.

We observe that for the VAG-CV scheme (since for all $\alpha \in \chi: \int_{\sigma} T_{f}\left(\llbracket \mathfrak{e}_{s^{\prime}} \rrbracket_{\mathcal{D}}^{\alpha}\right)\left(\llbracket \mathfrak{e}_{s} \rrbracket_{\mathcal{D}}^{\alpha}\right) \mathrm{d} \tau(\mathbf{x})=0$ for $s \neq s^{\prime}$ and $\left.\int_{\sigma} T_{f}\left(\llbracket \mathfrak{e}_{\sigma} \rrbracket_{\mathcal{D}}^{\alpha}\right)\left(\llbracket \mathfrak{e}_{s} \rrbracket_{\mathcal{D}}^{\alpha}\right) \mathrm{d} \tau(\mathbf{x})=0\right)$ as well as for the HFV scheme, the fluxes $F_{\nu_{m} \nu_{f}}$ are two point flux approximations.

The discrete source terms are defined by

$$
H_{\nu}= \begin{cases}\int_{\Omega} h_{m} \Pi_{\mathcal{D}_{m}} \mathfrak{e}_{\nu} \mathrm{d} \mathbf{x} & \text { for } \nu \in \operatorname{dof}_{\mathcal{D}_{m}}, \\ \int_{\Gamma} h_{f} \Pi_{\mathcal{D}_{f}} \mathfrak{e}_{\nu} \mathrm{d} \tau_{f}(\mathbf{x}) & \text { for } \nu \in d o f_{\mathcal{D}_{f}} .\end{cases}
$$

The following Finite Volume formulation of (2.2) is equivalent to the discrete variational formulation (3.1): find $\left(u_{\mathcal{D}_{m}}, u_{\mathcal{D}_{f}}\right) \in X_{\mathcal{D}}^{0}$ such that

$$
\begin{aligned}
& \text { for all } K \in \mathcal{M}: \sum_{\nu \in d o f_{K}} F_{K \nu}\left(u_{\mathcal{D}_{m}}\right)=H_{K} \\
& \text { for all } \sigma \in \mathcal{F}_{\Gamma}: \quad \sum_{\nu \in d o f_{\sigma}} F_{\sigma \nu}\left(u_{\mathcal{D}_{f}}\right)-\sum_{\substack{\nu_{m} \in d_{0} f_{\mathcal{D}_{m}} \\
\text { s.t. }\left(\nu_{m}, \sigma\right) \in \mathcal{C}}} F_{\nu_{m} \sigma}\left(u_{\mathcal{D}_{m}}, u_{\mathcal{D}_{f}}\right)=H_{\sigma} \\
& \text { for all } \nu_{m} \in d o f_{\mathcal{D}_{m}} \backslash\left(\mathcal{M} \cup d o f_{D i r_{m}}\right) \text { : } \\
& -\sum_{K \in \mathcal{M}_{\nu_{m}}} F_{K \nu_{m}}\left(u_{\mathcal{D}_{m}}\right)+\sum_{\substack{\nu_{f} \in d o f_{\mathcal{D}_{f}} \\
\text { s.t. }\left(\nu_{m}, \nu_{f}\right) \in \mathcal{C}}} F_{\nu_{m} \nu_{f}}\left(u_{\mathcal{D}_{m}}, u_{\mathcal{D}_{f}}\right)=H_{\nu_{m}}
\end{aligned}
$$

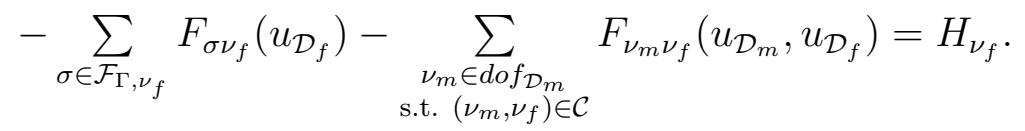



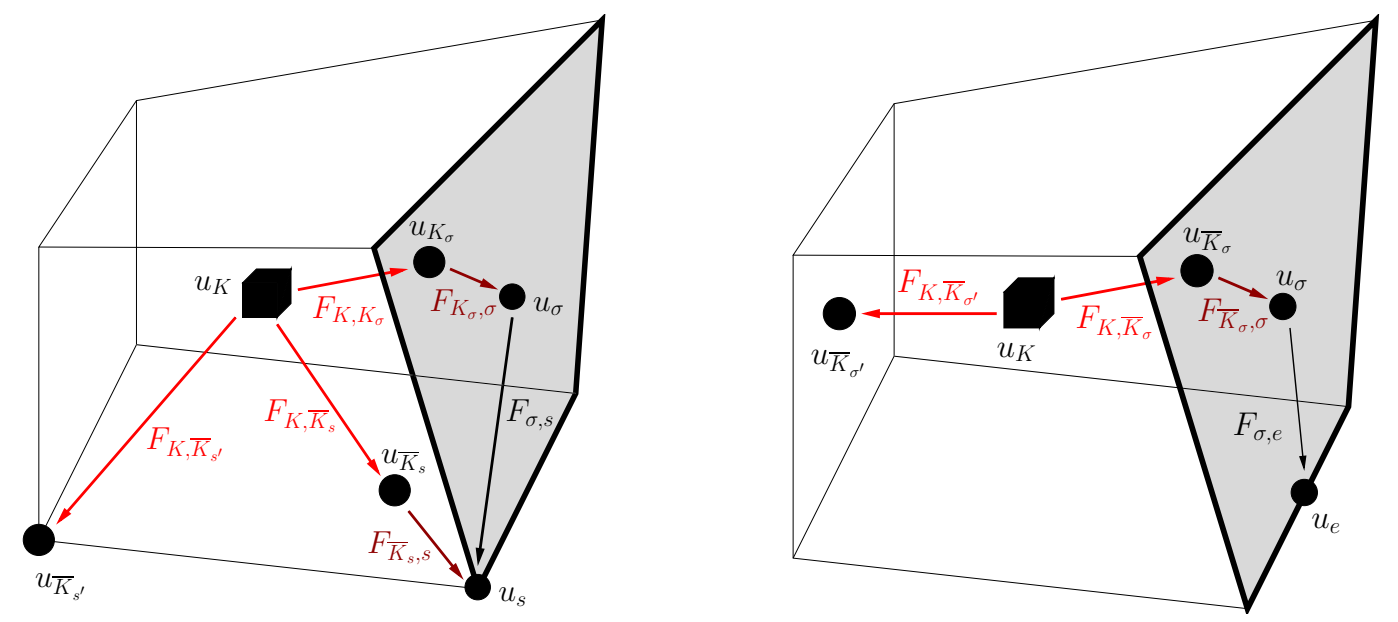

Figure 5: $m m$-fluxes (red), $m f$-fluxes (dark red) and ff-fluxes (black) for VAG (left) and HFV (right) on a 3D cell touching a fracture

Here, $\mathcal{M}_{\nu_{m}}$ stands for the set of indices $\left\{K \in \mathcal{M} \mid \nu_{m} \in d o f_{K}\right\}$ and $\mathcal{F}_{\Gamma, \nu_{f}}$ stands for the set $\left\{\sigma \in \mathcal{F}_{\Gamma} \mid \nu_{f} \in \operatorname{dof}_{\sigma}\right\}$.

It is important to note that, using the equation in each cell, the cell unknowns $u_{K}, K \in \mathcal{M}$, can be eliminated without fill-in. 